\title{
The gradient flow of O'Hara's knot energies
}

\author{
Simon Blatt ${ }^{1}(\mathbb{D}$
}

Received: 13 January 2016 / Revised: 13 March 2017 / Published online: 17 April 2017

(C) The Author(s) 2017. This article is an open access publication

\begin{abstract}
Jun O'Hara invented a family of knot energies $E^{j, p}, j, p \in(0, \infty)$, O'Hara in Topology Hawaii (Honolulu, HI, 1990). World Science Publication, River Edge 1992. We study the negative gradient flow of the sum of one of the energies $E^{\alpha}=E^{\alpha, 1}$, $\alpha \in(2,3)$, and a positive multiple of the length. Showing that the gradients of these knot energies can be written as the normal part of a quasilinear operator, we derive short time existence results for these flows. We then prove long time existence and convergence to critical points.
\end{abstract}

Mathematics Subject Classification 53C44 - 35S10

\section{Contents}

1 Introduction . . . . . . . . . . . . . . . . . . . . . . . . . . 994

2 Short time existence . . . . . . . . . . . . . . . . . . . . . . . . . 999

2.1 Quasilinear structure of the gradient . . . . . . . . . . . . . . . . . . 1000

2.2 Short time existence . . . . . . . . . . . . . . . . . . . . . . . . . . . . 1004

2.2 .1 The linear equation . . . . . . . . . . . . . . . . . . . . . 1006

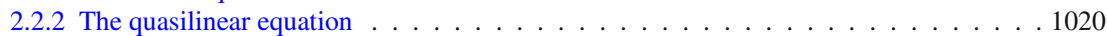

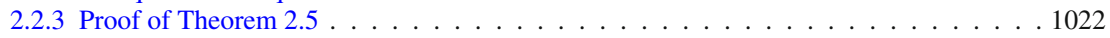

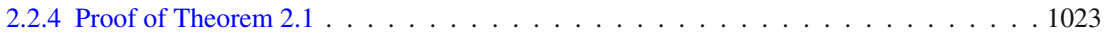

2.2.5 Proof of Theorem $2.2 \ldots \ldots \ldots \ldots 24 \ldots \ldots \ldots$

3 Long time existence . . . . . . . . . . . . . . . . . . . . . . . . . . . . . . . . . . . . . . . . . . . . . . . . . . . . . . . . . . . .

3.1 Coercivity of the energy . . . . . . . . . . . . . . . . . . . . . 1026

3.2 Evolution equations of higher order energies . . . . . . . . . . . . . . . . . 1028

Communicated by Y. Giga.

$凶$ Simon Blatt

simon.blatt@sbg.ac.at

1 Paris Lodron Universität Salzburg, Hellbrunner Strasse 34, 5020 Salzburg, Austria 
3.3 Interpolation estimates . . . . . . . . . . . . . . . . . . . . . 1030

3.4 Proof of long time existence . . . . . . . . . . . . . . . . . . . 1034

4 Asymptotics of the flows . . . . . . . . . . . . . . . . . . . . . . . . 1042

4.1 Łojasiewicz-Simon gradient estimate . . . . . . . . . . . . . . . . . . . . . . . . . . . . . . . . . . . . . . . . . . . . . . . . . . .

4.2 The flow above critical points . . . . . . . . . . . . . . . . . . . . . . . 1046

4.3 Completion of the Proof of Theorem 1.1 . . . . . . . . . . . . . . . . . . . 1052

5 Appendix: Analytic functions on Banach spaces . . . . . . . . . . . . . . . . . . . . . . 1052

6 Estimates for a generalization of the multilinear Hilbert transform . . . . . . . . . . . . . . . . . 1054

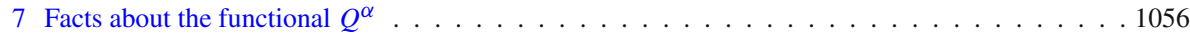

8 Interpolation and Leibniz rule for fractional Sobolev spaces . . . . . . . . . . . . . . . . . 1057

9 Normal graphs . . . . . . . . . . . . . . . . . . . . . . . . . . . . . 1059

References . . . . . . . . . . . . . . . . . . . . . . . 1060

\section{Introduction}

Is there an optimal way to tie a knot in Euclidean space? And if so, how nice are these optimal shapes? Is there a natural way to transform a given knot into this optimal shape?

To give a precise meaning to such questions a variety of energies for immersions have been invented and studied during the last twenty five years which are subsumed under the term knot energies.

In this article we deal with the third of the questions above. But first of all, let us gather some known answers to the first two questions.

The first family of geometric knot energies goes back to O'Hara. In [23], O'Hara suggested for $j, p \in(0, \infty)$ the energy

$$
E^{j, p}(c)=\iint_{(\mathbb{R} / \mathbb{Z})^{2}}\left(\frac{1}{|c(x)-c(y)|^{j}}-\frac{1}{d_{c}(x, y)^{j}}\right)^{p}\left|c^{\prime}(x)\right| \cdot\left|c^{\prime}(y)\right| d x d y
$$

of a regular closed curve $c \in C^{0,1}\left(\mathbb{R} / \mathbb{Z}, \mathbb{R}^{n}\right)$. Here, $d_{c}(x, y)$ denotes the distance of the points $x$ and $y$ along the curve $c$, i.e., the length of the shorter arc connecting these two points.

O'Hara observed that these energies are knot energies if and only if $j p>2[23$, Theorem 1.9] in the sense that then both pull-tight of a knot and selfintersections are punished. Furthermore, he showed that minimizers of the energies exist within every knot class if $j p>2$. So: Yes, there is an optimal way to tie a knot-actually even several ways to do so.

Abrams et al. proved in [1] that for $p \geq 1$ and $j p-1<2 p$ these energies are minimized by circles and that these energies are infinite for every closed regular curve if $j p-1 \geq 2 p .^{1}$

There is a reason why for the rest of our questions we will only consider the case $p=1$ : For $p \neq 1$, we expect that the first variation of $E^{j, p}$ leads to a degenerate elliptic operator of fractional order-even after breaking the symmetry of the equation

\footnotetext{
1 In fact one can even show that for $j p-1 \geq 2 p$ the energy is only finite for open curves that are part of a straight line-in which case the energy is 0 (cf. [9, Remark 1.3]).
} 
coming from the invariance under re-parameterizations. We will only consider the nondegenerate case $p=1$ and look at the one-parameter family

$$
E^{\alpha}(c):=E^{\alpha, 1}(c)=\iint_{(\mathbb{R} / \mathbb{Z})^{2}}\left(\frac{1}{|c(x)-c(y)|^{\alpha}}-\frac{1}{d_{c}(x, y)^{\alpha}}\right)\left|c^{\prime}(x)\right|\left|c^{\prime}(y)\right| d x d y .
$$

We leave the case $p \neq 1$ for a later study.

The most prominent member of this family is $E^{2}$ which is also known as Möbius energy due to the fact that it is invariant under Möbius transformations [14, Theorem 2.1]. While for $\alpha \in(2,3)$ the Euler-Lagrange equation is a non-degenerate elliptic sub-critical equation it is a critical equation for the case of the Möbius energy $E^{2}$.

In [14], Freedman, He, and Wang showed that even $E^{2}$ can be minimized within every prime knot class. Whether or not the same is true for composite knot classes is an open problem, though there are some numerical experiments that indicate that this might not be the case in every such knot class [19]. Furthermore, they derived a formula for the $L^{2}$-gradient of the Möbius energy [14, Equation 6.12] which was extended by Reiter [24, Theorem 1.45] to the energies $E^{\alpha}$ for $\alpha \in[2,3)$. They showed that the first variation of these functionals can be given by

$$
\delta_{h} E^{\alpha}(c):=\lim _{\varepsilon \rightarrow 0} \frac{E^{\alpha}(c+\varepsilon h)-E^{\alpha}(c)}{\varepsilon}=\int_{\mathbb{R} / \mathbb{Z}}\left\langle\mathfrak{V}^{\alpha}(c)(x), h(x)\right\rangle \cdot\left|c^{\prime}(x)\right| d x
$$

where

$$
\begin{aligned}
\mathfrak{V}^{\alpha}(c)(x):= & p \cdot v \cdot \int_{-\frac{1}{2}}^{\frac{1}{2}} P_{c^{\prime}(x)}^{\perp}\left\{2 \alpha \frac{c(x+w)-c(x)}{|c(x+w)-c(x)|^{2+\alpha}}-(\alpha-2) \frac{\kappa(x)}{d_{c}(x+w, x)^{\alpha}}\right. \\
& \left.-2 \frac{\kappa(x)}{|c(x+w)-c(x)|^{\alpha}}\right\}\left|c^{\prime}(x+w)\right| d w .
\end{aligned}
$$

Here, $P_{c^{\prime}}^{\perp}(u)=u-\left\langle u, \frac{c^{\prime}}{\left|c^{\prime}\right|}\right\rangle \frac{c^{\prime}}{\left|c^{\prime}\right|}$ denotes the orthogonal projection onto the normal part, and $\kappa=\kappa_{c}$ denotes the curvature vector of the curve $c$, i.e. $\kappa=\kappa_{c}=\left(\frac{d}{d s}\right)^{2} c$, where $\frac{d}{d s}=\frac{1}{\left|c^{\prime}\right|} \frac{d}{d x}$ is the derivative with respect to arc-length. Furthermore, $p \cdot v \int_{-\frac{1}{2}}^{\frac{1}{2}}=$ $\lim _{\varepsilon \downarrow 0} \int_{[-1 / 2,1 / 2] \backslash[-\varepsilon, \varepsilon]}$ denotes Cauchy's principal value.

In the case that $c$ is parameterized by arc length this reduces to

$$
\begin{aligned}
\mathfrak{V}^{\alpha}(c)(s):= & p . v \cdot \int_{-\frac{1}{2}}^{\frac{1}{2}} P_{c^{\prime}(x)}^{\perp}\left\{2 \alpha \frac{c(s+w)-c(s)}{|c(s+w)-c(s)|^{2+\alpha}}-(\alpha-2) \frac{c^{\prime \prime}(s)}{|w|^{\alpha}}\right. \\
& \left.-2 \frac{c^{\prime \prime}(s)}{|c(s+w)-c(s)|^{\alpha}}\right\} d w .
\end{aligned}
$$


Using the Möbius invariance of $E^{2}$, Freedman, He, and Wang showed that local minimizers of the Möbius energy are of class $C^{1,1}[14]$ —and thus gave a first answer to the question about the niceness of the optimal shapes. Zheng-Xu He combined this with a sophisticated bootstrapping argument to find that minimizers of the Möbius energy are of class $C^{\infty}$ [17]. Reiter could prove that critical points $c$ of $E^{\alpha}, \alpha \in(2,3)$ with $\kappa \in L^{\alpha}$ are smooth embedded curves [24], a result we extended to critical points of finite energy in [8] and to the Möbius energy in [9].

Let us now turn to the central theme of this article, the last of the three questions we started this article with: Is there a natural way to transform a given knot into its optimal shape? Since $E^{\alpha}$ is not scaling invariant for $\alpha \in(2,3)$, the $L^{2}$-gradient flow of $E^{\alpha}$ alone cannot have a nice asymptotic behavior. We want to avoid that the curve would get larger and larger in order to decrease the energy. Here, the length $L(c)$ of the curve $c$ will help us.

To transform a given knotted curve into a nice representative, we will look at the $L^{2}$-gradient flow of $E=E^{\alpha}+\lambda L$ for $\alpha \in(2,3)$ and $\lambda>0$ a fixed constant. This leads to the evolution equation

$$
\partial_{t} c=-\mathfrak{V}^{\alpha}(c)+\lambda \kappa_{c}
$$

Another Ansatz for a flow that might transform a given curve into a critical point is to let $\lambda$ depend on time in such a way that the length of the curve stays fixed during the flow. This leads to the evolution equation

$$
\partial_{t} c=-\mathfrak{V}^{\alpha}(c)+\lambda(t) \kappa
$$

where $\lambda(t)$ is given by

$$
\lambda(t)=-\frac{\int_{\mathbb{R} / l \mathbb{Z}}\left\langle\mathfrak{V}^{\alpha}(c)(x), \kappa(x)\left|c^{\prime}(x)\right| d x\right.}{\int_{\mathbb{R} / l \mathbb{Z}}|\kappa(x)|^{2}\left|c^{\prime}(x)\right| d x} .
$$

Unfortunately, the extra term is much harder to control than in the case for constant $\lambda$ due to its supercritical nature. We will not discuss this evolution equation further and leave it for a later study.

We will see that the right hand side of Eq. (1.4) can be written as the normal part of a quasilinear elliptic but non-local operator of order $\alpha+1 \in[3,4)$.

The main result of this article is the following theorem. Roughly speaking, it tells us that, given an initial regular embedded curve of class $C^{\infty}$, there exists a unique solution to the above evolution equations. This solution is immortal and converges to a critical point. More precisely we have:

Theorem 1.1 Let $\alpha \in(2,3)$ and $c_{0} \in C^{\infty}\left(\mathbb{R} / \mathbb{Z}, \mathbb{R}^{n}\right)$ be an injective regular curve. Then there is a unique smooth solution

$$
c \in C^{\infty}\left([0, \infty) \times \mathbb{R} / \mathbb{Z}, \mathbb{R}^{n}\right)
$$


to (1.4) with initial data $c(0)=c_{0}$ that after suitable re-parameterizations converges smoothly to a critical point of $E^{\alpha}+\lambda L$.

Note that we do not expect the above theorem to hold for the gradient flow of the Möbius energy $E^{2}$. Due to the criticality of the equation one gets in the case of the Möbius energy the long time behavior of this equation should be much richer. Numerical experiments yield to the conjecture that for example the gradient flow of the Möbius energy can develop singularities for certain initial data. It would make even less sense to consider the negative gradient flow of $E^{2}+\lambda L$ for a positive constant $\lambda$. As $E^{2}$ is scaling invariant the length term would force the flow to produce singularities in finite time.

Lin and Schwetlick showed similar results for the elastic energy plus some positive multiple of the Möbius energy and the length [20]. They succeeded in treating the term in the $L^{2}$-gradient coming from the Möbius energy as a lower order perturbation of the gradient of the elastic energy of curves. This allowed them to carry over the analysis due to Dziuk, Kuwert and Schätzle of the latter flow [12]. They proved long time existence for their flow and sub-convergence to a critical point up to re-parameterizations and translations.

The situation is quite different in the case we treat in this article. We have to understand the gradient of O'Hara's energies in a much more detailed way and have to use sharper estimates than in the work of Lin and Schwetlick. Furthermore, in contrast to Lin and Schwetlick we show that the complete flow, without going to a subsequence and applying suitable translations, converges to a critical point of our energy.

We proceed with an outline of the proof of Theorem 1.1 and thus with an outline of the paper. In Sect. 2, we prove short time existence results of this flow for initial data in little Hölder spaces and smooth dependence on the initial data. To do that, we show that the gradient of $E^{\alpha}$ is the normal part of an abstract quasilinear differential operator of fractional order (cf. Theorem 2.3). Combining Banach's fixed-point theorem with a maximal regularity result for the linearized equation, we get existence for a short amount of time. In order to keep this article as easily accessible as possible, we give a detailed proof of the necessary maximal regularity result.

The most important ingredient to the proof of long time existence in Sect. 3.4 is a strengthening of the classification of curves of finite energy $E^{\alpha}$ in [5] using fractional Sobolev spaces. For $s \in(0,1), p \in[1, \infty)$ and $k \in \mathbb{N}_{0}$ the space $W^{k+s, p}\left(\mathbb{R} / \mathbb{Z}, \mathbb{R}^{n}\right)$ consists of all functions $f \in W^{k, p}\left(\mathbb{R} / \mathbb{Z}, \mathbb{R}^{n}\right)$ for which

$$
\left|f^{(k)}\right|_{W^{s, p}}:=\left(\int_{\mathbb{R} / \mathbb{Z}} \int_{\mathbb{R} / \mathbb{Z}} \frac{\left|f^{(k)}(x)-f^{(k)}(y)\right|^{p}}{|x-y|^{1+s p}} d x d y\right)^{1 / p}
$$

is finite. This space is equipped with the norm $\|f\|_{W^{k+s, p}}:=\|f\|_{W^{k, p}}+\left|f^{(k)}\right|_{W^{s, p}}$. For a thorough discussion of the subject of fractional Sobolev space we point the reader to the monograph of Triebel [26]. Chapter 7 of [2] and the very nicely written and easy accessible introduction to the subject [13]. 
We know that a curve parameterized by arc length has finite energy $E^{\alpha}$ if and only if it is bi-Lipschitz and belongs to the space $W^{\frac{\alpha+1}{2}, 2}$. In Theorem 3.2 we show that even

$$
\left|c^{\prime}\right|_{W^{\frac{\alpha-1}{2}, 2}} \leq C E^{\alpha}
$$

and hence the $W^{\frac{\alpha+1}{2}}, 2$-norm of the flow is uniformly bounded in time.

To control higher order derivatives, we then derive the evolution equation of

$$
\mathcal{E}^{k}=\int_{\mathbb{R} / \mathbb{Z}}\left|\partial_{s}^{k} \kappa\right|^{2}\left|c^{\prime}(x)\right| d x .
$$

where $\kappa$ denotes the curvature of the curve $c$ and $\partial_{s}:=\frac{\partial_{x}}{\left|c^{\prime}(x)\right|}$ is the derivative with respect to the arc length parameter $s$. Note that in contrast to previous works like the work due to Dziuk, Kuwert, and Schätzle on the elastic flow or the work due to Lin and Schwetlick on the gradient flow of the elastic energy plus a positive multiple of the Möbius energy, we do not consider the normal derivatives of the curvature but the full derivatives with respect to arc length.

Using Gagliardo-Nirenberg-Sobolev inequalities for Besov spaces, which quite naturally appear during the calculation, together with a fractional Leibniz rule, we can then show that also the $\mathcal{E}^{k}$ are bounded uniformly in time. By standard arguments this will lead to long time existence and smooth subconvergence to a critical point after suitable translations and re-parameterization of the curves.

To get the full statement, we study the behavior of solutions near such critical points using a Łojasiewicz-Simon gradient estimate in Sect. 4. This allows us to show that flows starting close enough to a critical point and remaining above this critical point in the sense of the energy, exist for all time and converge to critical points. More precisely we have.

Theorem 1.2 (Long time existence above critical points) Let $c_{M} \in C^{\infty}\left(\mathbb{R} / \mathbb{Z}, \mathbb{R}^{n}\right)$ be a critical point of the energy $E=E^{\alpha}+\lambda L, \alpha \in(2,3), \lambda>0$, let $k \in \mathbb{N}, \delta>0$, and $\beta>\alpha$. Then there is a constant $\varepsilon>0$ such that the following is true:

Suppose that $\left(c_{t}\right)_{t \in[0, T)}$ is a maximal solution of the gradient flow of the energy $E$ with smooth initial data satisfying

$$
\left\|c_{0}-c_{M}\right\|_{C^{\beta}} \leq \varepsilon
$$

and

$$
E\left(c_{t}\right) \geq E\left(c_{M}\right)
$$

whenever there is a diffeomorphism $\phi_{t}: \mathbb{R} / \mathbb{Z} \rightarrow \mathbb{R} / \mathbb{Z}$ such that $\| c_{t} \circ \phi_{t}-$ $c_{M} \|_{C^{\beta}} \leq \delta$. Then the flow $\left(c_{t}\right)_{t}$ exists for all times and converges, after suitable re-parameterizations, smoothly to a critical point $c_{\infty}$ of $E$ satisfying

$$
E\left(c_{\infty}\right)=E\left(c_{M}\right)
$$


In contrast to Theorem 1.1, the analogue to Theorem 1.2 holds for the gradient flow of the Möbius energy alone [6, Theorem 5.1]. Note, that though the proof also works for the gradient flow of $E^{2}+\lambda L, \lambda \neq 0$, the statement would be empty as there are no critical points of this energy. This is due to the fact that $E^{2}$ is scaling invariant but $L$ is not. The same is true for the energies $E^{\alpha}-\lambda L, \lambda \geq 0$.

Theorem 1.2 shows that in the situation of Theorem 3.1 the complete solution converges to a critical point of $E^{\alpha}+\lambda L$ - even without applying any translations or re-parameterizations.

\section{Short time existence}

This section is devoted to an almost self-contained proof of short time existence for Eq. (1.4). We will show that for all $c_{0} \in C^{\infty}(\mathbb{R} / \mathbb{Z})$ a solution exists for some time.

For any space $X \subset C^{1}\left(\mathbb{R} / \mathbb{Z}, \mathbb{R}^{n}\right)$ we will denote by $X_{i r}$ the (open) subspace consisting of all injective (embedded) and regular curves in $X$.

Theorem 2.1 (Short time existence for smooth data) Let $c_{0} \in C_{i r}^{\infty}(\mathbb{R} / \mathbb{Z})$ and $\alpha \in$ $[2,3)$. Then there exists some $T=T\left(c_{0}\right)>0$ and a unique solution

$$
c \in C^{\infty}\left([0, T) \times \mathbb{R} / \mathbb{Z}, \mathbb{R}^{n}\right)
$$

of (1.4) with initial data $c(0, \cdot)=c_{0}$.

We can strengthen the result above. In fact, we can reduce the regularity of the initial curve even below the level where our evolution equation makes sense. We still can prove existence and in a sense also uniqueness of a family of curves with normal velocity given by the gradient of $E^{\alpha}+\lambda L$. For this purpose we will work in little Hölder spaces $h^{\beta}, \beta \notin \mathbb{N}$, which are the completion of $C^{\infty}$ with respect to the $C^{\beta}$ norm. We equip this space with the norm $\|\cdot\|_{h^{\beta}}=\|\cdot\|_{C^{\beta}}$. Let $\partial_{t}^{\perp} c$ denote the normal velocity, i.e. let $\partial_{t}^{\perp} c=\partial_{t} c-\frac{\left\langle\partial_{t} c, c^{\prime}\right\rangle}{\left|c^{\prime}\right|^{2}} c^{\prime}$.

Theorem 2.2 (Short time existence for non-smooth data) For $\alpha \in[2,3$ )

let $c_{0} \in h_{i, r}^{\beta}\left(\mathbb{R} / \mathbb{Z}, \mathbb{R}^{n}\right)$ for some $\beta>\alpha, \beta \notin \mathbb{N}$. Then there is a constant $T>0$ and a re-parameterization $\phi \in C^{\beta}(\mathbb{R} / \mathbb{Z}, \mathbb{R} / \mathbb{Z})$ such that there is a solution

$$
c \in C\left([0, T), h_{i, r}^{\beta}\left(\mathbb{R} / \mathbb{Z}, \mathbb{R}^{n}\right)\right) \cap C^{\infty}\left((0, T), C^{\infty}\left(\mathbb{R} / \mathbb{Z}, \mathbb{R}^{n}\right)\right)
$$

of the initial value problem

$$
\left\{\begin{array}{l}
\partial_{t}^{\perp} c=-\mathfrak{V}^{\alpha}(c)+\lambda \kappa_{c} \quad \forall t \in[0, T], \\
c(0)=c_{0} \circ \phi
\end{array}\right.
$$

This solution is unique in the sense that for each other solution

$$
\tilde{c} \in C\left([0, \tilde{T}), h_{i, r}^{\beta}\left(\mathbb{R} / \mathbb{Z}, \mathbb{R}^{n}\right)\right) \cap C^{\infty}\left((0, \tilde{T}), C^{\infty}\left(\mathbb{R} / \mathbb{Z}, \mathbb{R}^{n}\right)\right)
$$


and all $t \in(0, \min (T, \tilde{T})]$ there is a smooth diffeomorphism $\phi_{t} \in C^{\infty}(\mathbb{R} / \mathbb{Z}, \mathbb{R} / \mathbb{Z})$ such that

$$
c(t, \cdot)=\tilde{c}\left(t, \phi_{t}(\cdot)\right) .
$$

As in the special case of the Möbius energy dealt with in [6], these results are based on the fact that the functional $\mathfrak{V}^{\alpha}$ possesses a quasilinear structure-a statement that will be proven in the next subsection. After that we build a short time existence theory for the linearization of these equations from scratch and prove a maximal regularity result for this equation.

To get a solution of the evolution Eq. (1.4), we first have to break the symmetry that comes from the invariance of the equation under re-parameterizations. We do this by writing the time dependent family of curves $c$ as a normal graph over some fixed smooth curve $c_{0}$. Applying Banach's fixed-point theorem as done in [4] for nonlinear and quasilinear semiflows, we get short time existence for the evolution equation of the normal graphs and and continuous dependence of the solution on the initial data. A standard re-parameterization then gives Theorem 2.1 while Theorem 2.2 is obtained via an approximation argument.

\subsection{Quasilinear structure of the gradient}

As

$$
\kappa=\frac{1}{\left|c^{\prime}\right|} \frac{d}{d x}\left(\frac{c^{\prime}}{\left|c^{\prime}\right|}\right)=\frac{c^{\prime \prime}}{\left|c^{\prime}\right|^{2}}-\left\langle\frac{c^{\prime \prime}}{\left|c^{\prime}\right|^{2}}, \frac{c^{\prime}}{\left|c^{\prime}\right|} \frac{c^{\prime}}{\left|c^{\prime}\right|}=P_{c^{\prime}}^{\perp}\left(\frac{c^{\prime \prime}}{\left|c^{\prime}\right|^{2}}\right)\right.
$$

we can write

$$
\mathfrak{V}^{\alpha} c=P_{c^{\prime}}^{\perp} \tilde{\mathfrak{V}}^{\alpha} c
$$

where

$$
\begin{aligned}
\left(\tilde{\mathfrak{V}}^{\alpha} c\right)(x)= & p \cdot v \cdot \int_{-\frac{1}{2}}^{\frac{1}{2}}\left\{2 \alpha \frac{c(x+w)-c(x)-w c^{\prime}(x)}{|c(x+w)-c(x)|^{2+\alpha}}-(\alpha-2) \frac{c^{\prime \prime}(x)}{\left|c^{\prime}(x)\right|^{2} d_{c}(x+w, x)^{\alpha}}\right. \\
& \left.-2 \frac{c^{\prime \prime}(x)}{\left|c^{\prime}(x)\right|^{2}|c(x+w)-c(x)|^{\alpha}}\right\}\left|c^{\prime}(x+w)\right| d w .
\end{aligned}
$$

By exchanging every appearance of $|c(x+w)-c(x)|$ and $d_{c}(x+w, x)$ by their first order Taylor expansion $\left|c^{\prime}(x)\right||w|$ and $\left|c^{\prime}(x+w)\right|$ by $\left|c^{\prime}(x)\right|$ in the formula for $\tilde{\mathfrak{V}}^{\alpha}$, we are led to the conjecture that the leading order term of $\mathfrak{V}^{\alpha}$ is the normal part of $\frac{\alpha}{\left|c^{\prime}\right|^{\alpha+1}} Q^{\alpha}(c)$ where

$$
\left(Q^{\alpha} c\right)(x):=p . v . \int_{\left[-\frac{1}{2}, \frac{1}{2}\right]}\left(2 \frac{c(x+w)-c(x)-w c^{\prime}(x)}{w^{2}}-c^{\prime \prime}(x)\right) \frac{d w}{|w|^{\alpha}}
$$


is an operator of order $\alpha+1$ [24, Proposition 2.3], [17, Lemma 2.3].

This heuristic can be made rigorous using Taylor's expansions of the error terms and estimates for multilinear Hilbert transforms (cf. Lemma 6.2) leading to the next theorem. It will be essential later on that the remainder term is an analytic operator between certain function spaces_-which we denote by $C^{\omega}$. We denote by $H^{\beta}, \beta>2$, the Bessel potential spaces.

Theorem 2.3 (Quasilinear structure) For $\alpha \in(2,3)$ there is a mapping

$$
F^{\alpha} \in \bigcap_{\beta>0} C^{\omega}\left(C_{i, r}^{\alpha+\beta}\left(\mathbb{R} / \mathbb{Z}, \mathbb{R}^{n}\right), C^{\beta}\left(\mathbb{R} / \mathbb{Z}, \mathbb{R}^{n}\right)\right)
$$

such that

$$
\mathfrak{V}^{\alpha} c=\frac{\alpha}{\left|c^{\prime}\right|^{\alpha+1}} P_{c^{\prime}}^{\perp}\left(Q^{\alpha} c\right)+F^{\alpha} c
$$

for all $c \in H_{i, r}^{\alpha+1}\left(\mathbb{R} / \mathbb{Z}, \mathbb{R}^{n}\right)$.

Proof of Theorem 2.3 It is enough to show that there is a mapping

$$
\tilde{F}^{\alpha} \in \bigcap_{\beta>0} C^{\omega}\left(C_{i, r}^{\alpha+\beta}\left(\mathbb{R} / \mathbb{Z}, \mathbb{R}^{n}\right), C^{\beta}\left(\mathbb{R} / \mathbb{Z}, \mathbb{R}^{n}\right)\right)
$$

such that

$$
\tilde{\mathfrak{V}}^{\alpha} c=\frac{\alpha}{\left|c^{\prime}\right|^{\alpha+1}} Q^{\alpha} c+\tilde{F}^{\alpha} c
$$

for all $c \in H^{\alpha+1}\left(\mathbb{R} / \mathbb{Z}, \mathbb{R}^{n}\right)$. The theorem then follows immediately using $\mathfrak{V}^{\alpha} c=$ $P_{c^{\prime}}^{\perp}\left(\tilde{\mathfrak{V}}^{\alpha} c\right)=\frac{\alpha}{\left|c^{\prime}\right|^{\alpha+1}} P_{c^{\prime}}^{\perp}\left(Q^{\alpha} c\right)+\tilde{F}^{\alpha} c-\left\langle\tilde{F}^{\alpha} c, \frac{c^{\prime}}{\left|c^{\prime}\right|}\right\rangle \frac{c^{\prime}}{\left|c^{\prime}\right|}$.

We decompose

$$
\tilde{\mathfrak{V}}^{\alpha} c=\frac{\alpha}{\left|c^{\prime}\right|^{\alpha+1}} Q^{\alpha} c+2 \alpha R_{1}^{\alpha} c-(\alpha-2) R_{2}^{\alpha} c-2 R_{3}^{\alpha} c+\alpha R_{4}^{\alpha} c
$$

where

$$
\begin{aligned}
\left(R_{1}^{\alpha} c\right)(x):= & \int_{\mathbb{R} / \mathbb{Z}}\left(c(x+w)-c(x)-w c^{\prime}(x)\right) \\
& \times\left(\frac{1}{|c(x+w)-c(x)|^{\alpha+2}}-\frac{1}{\left|c^{\prime}(x)\right|^{\alpha+2}|w|^{\alpha+2}}\right)\left|c^{\prime}(x+w)\right| d w \\
\left(R_{2}^{\alpha} c\right)(x):= & \int_{\mathbb{R} / \mathbb{Z}} \frac{c^{\prime \prime}(x)}{\left|c^{\prime}(x)\right|^{2}}\left(\frac{1}{d_{c}(x+w, x)^{\alpha}}-\frac{1}{\left|c^{\prime}(x)\right|^{\alpha} w^{\alpha}}\right)\left|c^{\prime}(x+w)\right| d w, \\
\left(R_{3}^{\alpha} c\right)(x):= & \int_{\mathbb{R} / \mathbb{Z}} \frac{c^{\prime \prime}(x)}{\left|c^{\prime}(x)\right|^{2}}\left(\frac{1}{|c(x+w)-c(x)|^{\alpha}}-\frac{1}{\left|c^{\prime}(x)\right|^{\alpha} w^{\alpha}}\right)\left|c^{\prime}(x+w)\right| d w,
\end{aligned}
$$




$$
\begin{aligned}
\left(R_{4}^{\alpha} c\right)(x):= & \frac{1}{\left|c^{\prime}(x)\right|^{\alpha+2}} \int_{\mathbb{R} / \mathbb{Z}}\left(2 \frac{c(x+w)-c(x)-w c^{\prime}(x)}{w^{2}}\right. \\
& \left.-c^{\prime \prime}(x)\right) \frac{\left|c^{\prime}(x+w)\right|-\left|c^{\prime}(x)\right|}{|w|^{\alpha}} d w .
\end{aligned}
$$

Using Taylor's expansion up to first order, we get

$$
\begin{aligned}
d_{c}(x+w, w) & =|w| \int_{0}^{1}\left|c^{\prime}(x+\tau w)\right| d \tau \\
& =|w| \int_{0}^{1}\left\{\left|c^{\prime}(x)\right|+\tau w \int_{0}^{1}\left\langle\frac{c^{\prime}(x+s \tau w)}{\left|c^{\prime}(x+s \tau w)\right|}, c^{\prime \prime}(x+s \tau w)\right\rangle d s\right\} d \tau \\
& =|w|\left|c^{\prime}(x)\right|+w^{2} \int_{0}^{1}(1-\tau)\left\langle\frac{c^{\prime}(x+\tau w)}{\left|c^{\prime}(x+\tau w)\right|}, c^{\prime \prime}(x+\tau w)\right\rangle d \tau \\
& =|w|\left|c^{\prime}(x)\right|\left(1+w \tilde{X}_{c}(x, w)\right)
\end{aligned}
$$

where

$$
\tilde{X}_{c}(x, w):=\frac{1}{\left|c^{\prime}(x)\right|} \int_{0}^{1}(1-\tau)\left\langle\frac{c^{\prime}(x+\tau w)}{\left|c^{\prime}(x+\tau w)\right|}, c^{\prime \prime}(x+\tau w)\right\rangle d \tau
$$

and

$$
\begin{aligned}
|c(x+w)-c(x)|^{2} & =\left|w c^{\prime}(x)+w^{2} \int_{0}^{1}(1-\tau) c^{\prime \prime}(x+\tau w) d \tau\right|^{2} \\
& =w^{2}\left|c^{\prime}(x)\right|^{2}\left(1+w X_{c}(x, w)\right)
\end{aligned}
$$

where

$$
\begin{aligned}
X_{c}(x, w):= & \frac{1}{\left|c^{\prime}(x)\right|^{2}}\left(2\left\langle c^{\prime}(x), \int_{0}^{1}(1-\tau) c^{\prime \prime}(x+\tau w) d \tau\right\rangle\right. \\
& \left.+w\left|\int_{0}^{1}(1-\tau) c^{\prime \prime}(x+\tau w) d \tau\right|\right) .
\end{aligned}
$$


Together with the Taylor expansion

$$
|1+x|^{-\sigma}=1-\sigma x+\sigma(\sigma+1) x^{2} \int_{0}^{1}(1-\tau)|1+\tau x|^{-\sigma-2} d \tau
$$

for $\sigma>0$ and $x>-1$, this leads to

$$
\begin{aligned}
& \frac{1}{|c(x+w)-c(x)|^{\sigma}}-\frac{1}{\left|c^{\prime}(x)\right|^{\sigma}|w|^{\sigma}}=\frac{1}{\left|c^{\prime}(x)\right|^{\sigma}|w|^{\sigma}}\left(\left(1+w X_{c}(x, w)\right)^{-\frac{\sigma}{2}}-1\right) \\
& =\frac{1}{\left|c^{\prime}(x)\right|^{\sigma}|w|^{\sigma}}\left(-\frac{\sigma}{2} w X_{c}(x, w)\right. \\
& \left.\quad+\frac{\sigma}{2}\left(\frac{\sigma}{2}+1\right) w^{2} X_{c}(u, w)^{2} \int_{0}^{1}(1-\tau)\left(1+\tau w X_{c}(x, w)\right)^{-\frac{\sigma}{2}-2} d \tau\right)
\end{aligned}
$$

and

$$
\begin{aligned}
& \frac{1}{d(x+w, x)^{\sigma}}-\frac{1}{\left|c^{\prime}(x)\right|^{\sigma}|w|^{\sigma}}=\frac{1}{\left|c^{\prime}(u)\right|^{\sigma}|w|^{\sigma}} \\
& \times\left(-\sigma w \tilde{X}_{c}(x, w)+\sigma(\sigma+1) w^{2} \tilde{X}_{c}(u, w)^{2} \int_{0}^{1}(1-\tau)\left(1+\tau w \tilde{X}_{c}\right)^{-\sigma-2} d \tau\right) .
\end{aligned}
$$

Furthermore, we will use the identities

$$
c(x+w)-c(x)-w c^{\prime}(x)=w^{2} \int_{0}^{1}(1-\tau) c^{\prime \prime}(x+\tau w) d \tau
$$

and

$$
\left|c^{\prime}(x+w)\right|-\left|c^{\prime}(x)\right|=w \int_{0}^{1}\left\langle\frac{c^{\prime}(x+\tau w)}{\left|c^{\prime}(x+\tau w)\right|}, c^{\prime \prime}(x+\tau w)\right\rangle d \tau .
$$

Plugging these formulas into the expressions for the terms $R_{1}^{\alpha}, R_{2}^{\alpha}, R_{3}^{\alpha}, R_{4}^{\alpha}$ and factoring out, we see that they can be written as the sum of integrals as in the following Lemma 2.4. Hence, Lemma 2.4 completes the proof.

Lemma 2.4 For $\tilde{l}_{1} \leq l_{1}, \tilde{l}_{2} \leq l_{2}$, and $\tilde{l}_{3} \leq l_{3}$ and a multilinear operator $M$ let $I:=\int M\left(c^{\prime \prime}\left(x+\tau_{1} w\right), \ldots, c^{\prime \prime}\left(x+\tau_{l_{1}} w\right), c^{\prime}\left(x+\tau_{l_{1}+1} w\right), \ldots, c^{\prime}\left(x+\tau_{l_{1}+l_{2}} w\right)\right.$, $[0,1]^{\tilde{l}_{1}+\tilde{l}_{2}+\tilde{l}_{3}}$

$$
\begin{aligned}
& \left.\frac{c^{\prime}\left(x+\tau_{l_{1}+l_{2}+1} w\right)}{\left|c^{\prime}\left(x+\tau_{l_{1}+l_{2}+1} w\right)\right|}, \ldots, \frac{c^{\prime}\left(x+\tau_{l_{1}+l_{2}+l_{3}} w\right)}{\left|c^{\prime}\left(x+\tau_{l_{1}+l_{2}+l_{3}}\right)\right|}\right) \\
& \quad d \tau_{1} \cdots d \tau_{\tilde{l}_{1}} d \tau_{l_{1}+1} \cdots d \tau_{l_{1}+\tilde{l}_{2}} d \tau_{l_{1}+l_{2}+1} \cdots d \tau_{l_{1}+l_{2}+\tilde{l}_{3}},
\end{aligned}
$$


i.e. we integrate over some of the $\tau_{i}$ but not over all. Then for $\tilde{\alpha} \in(0,1)$ the functionals

$$
\begin{aligned}
& T_{1}(c)(x):=\int_{-1 / 2}^{1 / 2} \frac{I}{w|w|^{\tilde{\alpha}}} d w, \\
& \tilde{T}_{2}(c)(x):=\int_{-1 / 2}^{1 / 2} \frac{I\left(\int_{0}^{1}(1-\tau)\left(1+\tau w \tilde{X}_{c}(x, w)\right)^{-\sigma} d \tau\right)}{w|w|^{\tilde{\alpha}}} d w,
\end{aligned}
$$

and

$$
T_{2}(c)(x):=\int_{-1 / 2}^{1 / 2} \frac{I\left(\int_{0}^{1}(1-\tau)\left(1+\tau w X_{c}(x, w)\right)^{-\sigma}\right) d \tau}{|w|^{\tilde{\alpha}}} d w
$$

are analytic from $C^{\beta+\tilde{\alpha}+2}$ to $C^{\beta}$ for all $\beta>0$.

Proof The statement of the lemma for $T_{1}$ follows immediately from the boundedness of the multilinear Hilbert transform in Hölder spaces as stated in Remark 6.3 combined with the Lemmata 5.2 and 5.3.

Using a similar argument, one deduces that $c \rightarrow 1+\tau X_{c}$ is analytic, hence we get that for a given $c_{0}$ there is a neighborhood $U$ such that

$$
\left\|D_{c}^{m}\left(1+\tau X_{c}(\cdot, w)\right)\right\|_{L\left(C^{\beta+2}, C^{\beta}\right)} \leq C^{m} m !
$$

for all $c \in U$ where $C$ does not depend on $w$ and $\tau$. Using that $v \rightarrow|v|^{\sigma}$ is analytic away from 0 , we deduce that

$$
\left\|D_{c}^{m}\left(1+\tau X_{c}(\cdot, w)\right)^{\sigma}\right\|_{L\left(C^{\beta+2}, C^{\beta}\right)} \leq C^{m} m !
$$

using Lemma 5.2 and the fact that the composition of analytic functions is analytic. Hence, the integrands in the definitions of $T_{2}$ and by the same argument also of $\tilde{T}_{2}$ satisfy the assumptions of Lemma 5.3. Hence, $T_{2}$ and $\tilde{T}_{2}$ are even analytic operators from $C^{\beta+2}\left(\mathbb{R} / \mathbb{Z}, \mathbb{R}^{n}\right)$ to $C^{\beta}\left(\mathbb{R} / \mathbb{Z}, \mathbb{R}^{n}\right)$.

\subsection{Short time existence}

Using the quasilinear form of $\mathfrak{V}^{\alpha}$, we derive short time existence results for the gradient flow of O'Hara's energies in this section. For this task, we will work with families of curves that are normal graphs over a fixed smooth curve $c_{0}$ and whose normal part belongs to a small neighborhood of 0 in $h^{\beta}, \beta>\alpha$.

To describe these neighborhoods, note that there is a strictly positive, lower semicontinuous function $r: C_{i, r}^{2}\left(\mathbb{R} / \mathbb{Z}, \mathbb{R}^{n}\right) \rightarrow(0, \infty)$ such that

$$
c+\left\{N \in C^{2}\left(\mathbb{R} / \mathbb{Z}, \mathbb{R}^{n}\right)_{c}^{\perp}:\|N\|_{C^{2}}<r(c)\right\}
$$


only contains regular embedded curves for all $c \in C_{i, r}^{2}\left(\mathbb{R} / \mathbb{Z}, \mathbb{R}^{n}\right)$ and

$$
r(c) \leq 1 / 2 \inf _{x \in \mathbb{R} / \mathbb{Z}}\left|c^{\prime}(x)\right| .
$$

Here, $C^{\beta}\left(\mathbb{R} / \mathbb{Z}, \mathbb{R}^{n}\right)_{c}^{\perp}$ denotes the space of all vector fields $N \in C^{\beta}\left(\mathbb{R} / \mathbb{Z}, \mathbb{R}^{n}\right)$ which are normal to $c$, i.e. for which $\left\langle c^{\prime}(u), N(u)\right\rangle=0$ for all $u \in \mathbb{R} / \mathbb{Z}$. Letting

$$
\mathcal{V}_{r, \beta}(c):=\left\{N \in h^{\beta}\left(\mathbb{R} / \mathbb{Z}, \mathbb{R}^{n}\right)_{c}^{\perp}:\|N\|_{C^{1}}<r(c)\right\}
$$

we have for all $c \in h_{i, r}^{\beta}\left(\mathbb{R} / \mathbb{Z}, \mathbb{R}^{n}\right)$

$$
c+\mathcal{V}_{r, \beta}(c) \subset h_{i, r}^{\beta}\left(\mathbb{R} / \mathbb{Z}, \mathbb{R}^{n}\right)
$$

Let $N \in \mathcal{V}_{r, \beta}(c)$. Equation (2.3) guarantees that $P_{(c+N)^{\prime}(u)}^{\perp}$ is an isomorphism from the normal space along $c$ at $u$ to the normal space along $c+N$. Otherwise there would be a $v \neq 0$ in the normal space of $c$ at $u$ such that

$$
0=P_{(c+N)^{\prime}(u)}^{\perp}(v)=v-\left\langle v, \frac{(c+N)^{\prime}(u)}{\left|(c+N)^{\prime}(u)\right|}\right\rangle \frac{(c+N)^{\prime}(u)}{\left|(c+N)^{\prime}(u)\right|}
$$

which would contradict

$$
\left|v-\left\langle v, \frac{(c+N)^{\prime}(u)}{\left|(c+N)^{\prime}(u)\right|}\right\rangle \frac{(c+N)^{\prime}(u)}{\left|(c+N)^{\prime}(u)\right|}\right| \geq|v|-\left|\left\langle v, \frac{N^{\prime}(u)}{\left|(c+N)^{\prime}(u)\right|}\right\rangle\right| \geq|v| / 4>0 .
$$

For $c \in C^{1}\left((0, T), C_{i, r}^{1}\left(\mathbb{R} / \mathbb{Z}, \mathbb{R}^{n}\right)\right)$ we denote by

$$
\partial_{t}^{\perp} c=P_{c^{\prime}}^{\perp}\left(\partial_{t} c\right)
$$

the normal velocity of the family of curves.

We prove the following strengthened version of the short time existence result mentioned at the beginning of Sect. 2 .

Theorem 2.5 (Short time existence for normal graphs) Let $c_{0} \in C^{\infty}\left(\mathbb{R} / \mathbb{Z}, \mathbb{R}^{n}\right)$ be an embedded regular curve, $\alpha \in[2,3)$, and $\beta>\alpha, \beta \notin \mathbb{N}$.

Then for every $N_{0} \in \mathcal{V}_{r, \beta}\left(c_{0}\right)$ there is a constant $T=T\left(N_{0}\right)>0$ and a neighborhood $U \subset \mathcal{V}_{r, \beta}\left(c_{0}\right)$ of $N_{0}$ such that for every $\tilde{N}_{0} \in U$ there is a unique solution $N_{\tilde{N}_{0}} \in C\left([0, T), h^{\beta}(\mathbb{R} / \mathbb{Z})_{c_{0}}^{\perp}\right) \cap C^{1}\left((0, T), C^{\infty}(\mathbb{R} / \mathbb{Z})_{c_{0}}^{\perp}\right)$ of

$$
\left\{\begin{array}{l}
\partial_{t}^{\perp}\left(c_{0}+N\right)=-\mathfrak{V}^{\alpha}\left(c_{0}+N\right)+\lambda \kappa_{c_{0}+N} \quad t \in[0, T] \\
N(0)=\tilde{N}_{0}
\end{array}\right.
$$

Furthermore, the flow $\left(\tilde{N}_{0}, t\right) \mapsto N_{\tilde{N}_{0}}(t)$ is in $C^{\infty}\left((U \times(0, T)), C^{\infty}(\mathbb{R} / \mathbb{Z})\right)$. 
The proof of Theorem 2.5 consists of two steps. First we show that (2.5) can be transformed into an abstract quasilinear system of parabolic type. The second step is to establish short time existence results for the resulting equation.

The second step can be done using general results about analytic semigroups, regularity of pseudo-differential operators with rough symbols [7], and the short time existence results for quasilinear equations in [4] or [3]. Furthermore, we need continuous dependence of the solution on the data and smoothing effects in order to derive the long time existence results in Sect. 4.

For the convenience of the reader, we go a different way here and present a selfcontained proof of the short time existence that only relies on a characterization of the little Hölder spaces as trace spaces. In Sect. 2.2.1, we deduce a maximal regularity result for solutions of linear equations of type $\partial_{t} u+a(t) Q^{s-1} u+b(t) u=f$ in little Hölder spaces using heat kernel estimates. Following ideas from [4], we then prove short time existence and differentiable dependence on the data for the quasilinear equation.

\subsubsection{The linear equation}

We will derive a priori estimates and existence results for linear equations of the type

$$
\left\{\begin{array}{l}
\partial_{t} u+a Q^{s-1} u+b u=f \text { in } \mathbb{R} / \mathbb{Z} \times(0, T) \\
u(0)=u_{0}
\end{array}\right.
$$

using little Hölder spaces, where $b(t) \in L\left(h^{\beta}\left(\mathbb{R} / \mathbb{Z}, \mathbb{R}^{n}\right), h^{\beta}\left(\mathbb{R} / \mathbb{Z}, \mathbb{R}^{n}\right)\right)$ and $a(t) \in$ $h^{\beta}(\mathbb{R} / \mathbb{Z},(0, \infty)), s \in[3.4)$.

For $\theta \in(0,1), \beta>0$, and $T>0$ we will consider solutions that lie in the space

$$
\begin{array}{r}
X_{T}^{\theta, \beta}:=\left\{g \in C ( ( 0 , T ) , h ^ { \beta + s } ( \mathbb { R } / \mathbb { Z } , \mathbb { R } ^ { n } ) ) \cap C ^ { 1 } \left((0, T), h^{\beta}\left(\mathbb{R} / \mathbb{Z}, \mathbb{R}^{n}\right):\right.\right. \\
\left.\sup _{t \in(0, T)} t^{1-\theta}\left(\left\|\partial_{t} g(t)\right\|_{C^{\beta}}+\|g(t)\|_{C^{s+\beta}}\right)<\infty\right\}
\end{array}
$$

and equip this space with the norm

$$
\|g\|_{X_{T}^{\theta, \beta}}:=\sup _{t \in(0, T)} t^{1-\theta}\left(\left\|\partial_{t} g(t)\right\|_{C^{\beta}}+\|g(t)\|_{C^{s+\beta}}\right) .
$$

The right hand side $f$ of our equation should then belong to the space

$$
Y_{T}^{\theta, \beta}:=\left\{g \in C\left((0, T), h^{\beta}\left(\mathbb{R} / \mathbb{Z}, \mathbb{R}^{n}\right)\right): \sup _{t \in(0, T)} t^{1-\theta}\|g(t)\|_{C^{\beta}}<\infty\right\}
$$

equipped with the norm

$$
\|g\|_{Y_{T}^{\theta, \beta}}:=\sup _{t \in(0, T)} t^{1-\theta}\|g(t)\|_{C^{\beta}} .
$$


From the trace method in the theory of interpolation spaces (cf. [21, Section 1.2.2]), the following relation of the space $X_{\theta, \beta}$ to the little Hölder space $h^{\beta+s \theta}$ is well known if $\beta+s \theta$ is not an integer:

If $u \in X_{T}^{\theta, \beta}$ then $u(t)$ converges in $h^{\beta+s \theta}$ to a function $u(0)$ as $t \searrow 0$ with

$$
\|u(0)\|_{C^{\beta+s \theta}} \leq C\|u\|_{X_{T}^{\theta, \beta}} .
$$

On the other hand, for every $u_{0} \in h^{\beta+s \theta}$ there is a $u \in X_{T}^{\theta, \beta}$ such that $u(t)$ converges in $h^{\beta+s \theta}$ to $u(0)$ for $t \rightarrow 0$ and

$$
\|u\|_{X_{T}^{\theta, \beta}} \leq\left\|u_{0}\right\|_{C^{\beta+s \theta}}
$$

Given $u_{0}$ we will see that the solution of the initial value problem

$$
\begin{cases}\partial_{t} u+(-\Delta)^{s / 2} u=0 & \text { on }(0, \infty) \times \mathbb{R} \\ u=u_{0} & \text { at } t=0\end{cases}
$$

satisfies (2.7). The well-known embedding $X_{T}^{\theta, \beta} \subset C^{\theta}\left((0, T), C^{\beta}\left(\mathbb{R} / \mathbb{Z}, \mathbb{R}^{n}\right)\right)$ will also be essential in the proof (cf. [21, p. 20].

The aim of this subsection is to prove the following theorem about the solvability of our linear equation:

Theorem 2.6 Let $T>0, \beta>0, \theta \in(0,1)$ with $\beta+s \theta \notin \mathbb{N}$, and $a \in C^{1}\left([0, T], h^{\beta}(\mathbb{R} / \mathbb{Z},[0, \infty))\right), \quad b \in C^{0}\left((0, T), L\left(h^{\beta}\left(\mathbb{R} / \mathbb{Z}, \mathbb{R}^{n}\right), h^{\beta}\left(\mathbb{R} / \mathbb{Z}, \mathbb{R}^{n}\right)\right)\right)$ with

$$
\|a\|_{C^{1}\left([0, T], C^{\beta}\right)}+\sup _{t \in(0, T)} t^{1-\theta}\|b(t)\|_{L\left(h^{\beta}, h^{\beta}\right)}<\infty
$$

Then the mapping $J: u \mapsto\left(u(0), \partial_{t} u+a Q^{s-1} u+b u\right)$ defines an isomorphism between $X_{T}^{\theta, \beta}$ and $h^{\beta+\theta s}\left(\mathbb{R} / \mathbb{Z}, \mathbb{R}^{n}\right) \times Y_{T}^{\theta, \beta}$.

This will be enough to prove short time existence of a solution for some quasilinear equations later on using Banach's fixed-point theorem.

Equation (2.6) already guarantees that $J$ is a bounded linear operator. So we only have to prove that it is onto for which we will use some a priori results (also called maximal regularity results in this context) together with the method of continuity.

To derive these estimates, we will freeze the coefficients and use a priori estimates for $\partial_{t} u+\lambda(-\Delta)^{s / 2} u=f$ on $\mathbb{R}$ where $\lambda>0$ is a constant. He observed in [16], that the fractional Laplacian can be expressed by

$$
c_{s}(-\Delta)^{s / 2} u=p \cdot v \cdot \int_{-\infty}^{\infty}\left(2 \frac{u(x+w)-u(x)-w u^{\prime}(x)}{|w|^{2}}-u^{\prime \prime}(x)\right) \frac{d w}{|w|^{s-1}}
$$


for a $c_{s}>0$ for all $u \in H^{s}\left(\mathbb{R}, \mathbb{R}^{n}\right)$. We will use this identity together with a localization argument to get from $(-\Delta)^{s / 2}$ living on $\mathbb{R}$ back to our operator $Q^{s-1}$ which lives on the circle $\mathbb{R} / \mathbb{Z}$.

Note that the fractional Laplacian $(-\Delta)^{s / 2}$ on $\mathbb{R}$ is bounded from $C_{0}^{s+\beta}\left(\mathbb{R}, \mathbb{R}^{n}\right)$, the space of all $C^{s+\beta}$ function with compact support, to $C^{\beta}\left(\mathbb{R}, \mathbb{R}^{n}\right)$ for all $\beta>0$, $\beta \notin \mathbb{N}$.

Let us consider the heat kernel of the equation $\partial_{t} u+(-\Delta)^{s / 2} u=0$ which is given by

$$
G_{t}(x):=\frac{1}{2 \pi} \int_{\mathbb{R}} e^{2 \pi i k x} e^{-t|2 \pi k|^{s}} d k .
$$

for all $t>0$ and $x \in \mathbb{R}$.

Since $k \mapsto e^{-t|2 \pi k|^{s}}$ is a Schwartz function, its inverse Fourier transform $G_{t}$ is a Schwartz function as well. Furthermore, one easily sees using the Fourier transformation that

$$
\partial_{t} G_{t}+(-\Delta)^{s / 2} G_{t}=0 \text { on } \mathbb{R} \quad \forall t>0 .
$$

The most important property for us is the scaling

$$
G_{t}(x)=t^{-1 / s} G_{1}\left(t^{-1 / s} x\right),
$$

from which we deduce

$$
\partial_{x}^{k} G_{t}(x)=t^{-(1+k) / s}\left(\partial_{x}^{k} G_{1}\right)\left(t^{-1 / s} x\right)
$$

and hence

$$
\left\|\partial_{x}^{k} G_{t}\right\|_{L^{1}(\mathbb{R})} \leq C_{k} t^{-k / s}\left\|\partial_{x}^{k} G_{1}\right\|_{L^{1}(\mathbb{R})} \leq C_{k} t^{-k / s} .
$$

Combining these relations with standard interpolation techniques, we get the following estimates for the heat kernel

Lemma 2.7 (Heat kernel estimates) For all $0 \leq \beta_{1} \leq \beta_{2}$, and $T>0$ there is $a$ constant $C=C\left(\beta_{1}, \beta_{2}, T\right)<\infty$ such that

$$
\left\|G_{t} * f\right\|_{C^{\beta_{2}}} \leq C t^{-\left(\beta_{2}-\beta_{1}\right) / s}\|f\|_{C^{\beta_{1}}} \quad \forall f \in C^{\beta_{1}}\left(\mathbb{R}, \mathbb{R}^{n}\right), t \in(0, T] .
$$

Proof Let $k_{i} \in \mathbb{N}_{0}$ and $\tilde{\beta}_{i} \in[0,1)$ be such that $\beta_{i}=k_{i}+\tilde{\beta}_{i}$ for $i=1,2$.

For $l>m, l, m \in \mathbb{N}_{0}$ and $\beta \in(0,1)$, we deduce from $\int_{\mathbb{R}} \partial_{x}^{l-m} G_{t}(y) d y=0$ and the fact that $G_{1}$ is a Schwartz function

$$
\begin{aligned}
&\left|\partial_{x}^{l}\left(G_{t} * f\right)(x)\right|=\left|\int_{\mathbb{R}} \partial_{x}^{l-m} G_{t}(y)\left(\partial_{x}^{m} f(x-y)-\partial_{x}^{m} f(x)\right) d y\right| \\
& \stackrel{(2.12)}{\leq} \int_{\mathbb{R}} t^{-(1+l-m) / s}\left|\left(\partial_{x}^{l-m} G_{1}\right)\left(y / t^{1 / s}\right)\right| \cdot\left|\left(\partial_{x}^{m} f(x-y)-\partial_{x}^{m} f(x)\right)\right| d y
\end{aligned}
$$




$$
\begin{aligned}
\left.\stackrel{z=}{y / t^{1 / s}} \int_{\mathbb{R}} t^{-(l-m) / s}\left|\left(\partial_{x}^{l-m} G_{1}\right)(z)\right| \cdot \mid \partial_{x}^{m} f\left(x-t^{1 / s} z\right)-\partial_{x}^{m} f(x)\right) \mid d z \\
\leq t^{-(l-(m+\beta)) / s} \operatorname{höl}_{\beta}\left(\partial_{x}^{m} f\right) \int_{\mathbb{R}}\left|\left(\partial_{x}^{l-m} G_{1}\right)(z)\right||z|^{\beta} d z \\
\quad \leq C_{l, m, \beta} t^{-(l-(m+\beta)) / s} \operatorname{höl}_{\beta}\left(\partial_{x}^{m} f\right) .
\end{aligned}
$$

For all $l \geq m, \beta \in(0,1)$ we have

$$
\operatorname{höl}_{\beta}\left(\partial_{x}^{l}\left(G_{t} * f\right)\right) \leq C_{l-m} t^{-(l-m) / s} \operatorname{höl}_{\beta}\left(\partial_{x}^{m} f\right)
$$

as for all $x_{1}, x_{2} \in \mathbb{R}$

$$
\begin{aligned}
\mid \partial_{x}^{l}\left(G_{t} * f\right)\left(x_{1}\right) & -\partial_{x}^{l}\left(G_{t} * f\right)\left(x_{2}\right) \mid \\
& =\left|\int_{\mathbb{R}} \partial_{x}^{l-m} G_{t}(y)\left(\partial_{x}^{m} f\left(x_{1}-y\right)-\partial_{x}^{m} f\left(x_{2}-y\right)\right) d y\right| \\
& \leq\left\|\partial_{x}^{l-m} G_{t}\right\|_{L^{1}} \operatorname{höl}_{\beta}\left(\partial_{x}^{m} f\right)\left|x_{1}-x_{2}\right|^{\beta} \\
& \stackrel{(2.13)}{\leq} C_{l-m} t^{-(l-m) / s} \operatorname{höl}_{\beta}\left(\partial_{x}^{m} f\right)\left|x_{1}-x_{2}\right|^{\beta} .
\end{aligned}
$$

In a similar way we obtain for all $l \geq m$

$$
\left\|\partial_{x}^{l}\left(G_{t} * f\right)\right\|_{L^{\infty}} \leq C_{l-m} t^{-(l-m) / s}\left\|\partial_{x}^{m} f\right\|_{L^{\infty}} .
$$

Combining these three estimates, we get

$$
\begin{aligned}
\left\|G_{t} * f\right\|_{C^{k_{2}+\tilde{\beta}_{1}}} & \leq C t^{-\left(k_{2}-k_{1}\right) / s}\|f\|_{C^{k_{1}+\tilde{\beta}_{1}}}, \\
\left\|G_{t} * f\right\|_{C^{k_{2}+1}} & \leq C t^{-\left(\left(k_{2}+1\right)-\left(k_{1}+\tilde{\beta}_{1}\right)\right) / s}\|f\|_{C^{k_{1}+\tilde{\beta}_{1}}},
\end{aligned}
$$

and if $k_{2}>k_{1}$

$$
\left\|G_{t} * f\right\|_{C^{k_{2}}} \leq C t^{-\left(k_{2}-\left(k_{1}+\tilde{\beta}_{1}\right)\right) / s}\|f\|_{C^{k_{1}+\tilde{\beta}_{1}}}
$$

Furthermore, we will use that for $0 \leq \alpha \leq \beta \leq \gamma \leq 1, \alpha \neq \gamma$ and $f \in C^{\gamma}$ we have the interpolation inequality

$$
\|f\|_{C^{\beta}} \leq 2\|f\|_{C^{\gamma}}^{\frac{\beta-\alpha}{\gamma-\alpha}}\|f\|_{C^{\alpha}}^{\frac{\gamma-\beta}{\gamma-\alpha}}
$$


which can in the case of $\alpha>0$ be obtained from

$$
\begin{aligned}
\left|f\left(x_{1}\right)-f\left(x_{2}\right)\right| & \leq\left|f\left(x_{1}\right)-f\left(x_{2}\right)\right|^{\frac{\beta-\alpha}{\gamma-\alpha}}\left|f\left(x_{1}\right)-f\left(x_{2}\right)\right|^{\frac{\gamma-\beta}{\gamma-\alpha}} \\
& \leq\left(\operatorname{hö}{ }_{\gamma}(f)\left|x_{1}-x_{2}\right|^{\gamma}\right)^{\frac{\beta-\alpha}{\gamma-\alpha}}\left(\operatorname{höl}_{\alpha}(f)\left|x_{1}-x_{2}\right|^{\alpha}\right)^{\frac{\gamma-\beta}{\gamma-\alpha}}
\end{aligned}
$$

and in the case that $\alpha=0$ from

$$
\begin{aligned}
\left|f\left(x_{1}\right)-f\left(x_{2}\right)\right| & \leq\left|f\left(x_{1}\right) f\left(x_{2}\right)\right|^{\frac{\beta}{\gamma}}\left|f\left(x_{1}\right)-f\left(x_{2}\right)\right|^{\frac{\gamma-\beta}{\gamma}} \\
& \leq\left(\operatorname{höl}_{\gamma}(f)\left|x_{1}-x_{2}\right|^{\gamma}\right)^{\frac{\beta}{\gamma}}\left(2\|f\|_{L^{\infty}}\right)^{\frac{\gamma-\beta}{\gamma}} .
\end{aligned}
$$

For $\tilde{\beta}_{2} \geq \tilde{\beta}_{1}$ we get

$$
\begin{gathered}
\left\|G_{t} * f\right\|_{C^{k_{2}+\tilde{\beta}_{2}}} \leq C\left(\left\|\partial_{x}^{k_{2}}\left(G_{t} * f\right)\right\|_{C^{\tilde{\beta}_{2}}}+\left\|G_{t} * f\right\|_{L^{\infty}}\right) \\
\stackrel{(2.17)}{\leq} C\left(\left\|\partial_{x}^{k_{2}}\left(G_{t} * f\right)\right\|_{C^{\tilde{\beta}_{1}}}^{\frac{1-\tilde{\beta}_{2}}{1-\tilde{\beta}_{1}}}\left\|\partial_{x}^{k_{2}+1}\left(G_{t} * f\right)\right\|_{C^{0}}^{\frac{\tilde{\beta}_{2}-\tilde{\beta}_{1}}{1-\tilde{\beta}_{1}}}+\|f\|_{\left.L^{\infty}\right)}\right. \\
\stackrel{(2.14) \&(2.15)}{\leq} C\left(t^{-\left(\beta_{2}-\beta_{1}\right) / s}+1\right)\|f\|_{C^{\beta_{1}}} .
\end{gathered}
$$

For $\tilde{\beta}_{1}>\tilde{\beta}_{2}$ and hence $k_{1}<k_{2}$, we obtain

$$
\begin{gathered}
\left\|G_{t} * f\right\|_{C^{k_{2}+\tilde{\beta}_{2}}} \leq C\left(\left\|\partial_{x}^{k_{2}}\left(G_{t} * f\right)\right\|_{C^{\tilde{\beta}_{2}}}+\left\|G_{t} * f\right\|_{L^{\infty}}\right) \\
\stackrel{(2.17)}{\leq} C\left(\left\|\partial_{x}^{k_{2}}\left(G_{t} * f\right)\right\|_{C^{\tilde{\beta}_{1}}}^{\tilde{\beta}_{2}}\left\|\partial_{x}^{\tilde{\beta}_{2}}\left(G_{t} * f\right)\right\|_{C^{0}}^{\frac{\tilde{\beta}_{1}-\tilde{\beta}_{2}}{\tilde{\beta}_{1}}}+\left\|G_{t} * f\right\|_{\left.L^{\infty}\right)}\right. \\
\stackrel{(2.14) \&(2.16)}{\leq} C\left(t^{-\left(\beta_{2}-\beta_{1}\right) / s}+1\right)\|f\|_{C^{\beta_{1}} .} .
\end{gathered}
$$

To derive a representation formula for the solution of $\partial_{t} u+(-\Delta)^{s / 2} u=f$, we need the following simple fact

Lemma 2.8 For all $t>0$ we have

$$
\int_{\mathbb{R}} G_{t}(x) d x=1 .
$$

Furthermore, for all $f \in h^{\beta}\left(\mathbb{R}, \mathbb{R}^{n}\right), \beta \notin \mathbb{N}$, there holds

$$
G_{t} * f \stackrel{t \downarrow 0}{\longrightarrow} f \quad \text { in } h^{\beta}\left(\mathbb{R}, \mathbb{R}^{n}\right) .
$$

Proof For $g \in L^{2}(\mathbb{R})$ let $\hat{g}$ denote the Fourier transform of $g$. 
For $t>0$ and $f \in L^{2}(\mathbb{R})$ we obtain from Lebesgue's theorem of dominated convergence

$$
\left(G_{t} * f\right)^{\wedge}=e^{-t|2 \pi \cdot|^{s}} \hat{f} \stackrel{t \downarrow 0}{\longrightarrow} \hat{f} \quad \text { in } L^{2} .
$$

Hence, Plancherel's formula shows

$$
G_{t} * f \rightarrow f \quad \text { in } L^{2}
$$

Setting $f=\chi_{[-1,1]}$ and observing

$$
\lim _{t \searrow 0}\left(G_{t} * f\right)(x)=\lim _{t \searrow 0} \int_{\left[t^{-1 / s}(x-1), t^{-1 / s}(x+1)\right]} G_{1} d y=\int_{\mathbb{R}} G_{1} d y, \quad \forall x \in(-1,1),
$$

we deduce that

$$
\int_{\mathbb{R}} G_{1} d y=1
$$

To prove the second part, let $f \in h^{\beta}\left(\mathbb{R}, \mathbb{R}^{n}\right)$. From convergence results for smoothing kernels we get for all $\tilde{f} \in C^{\infty}(\mathbb{R})$

$$
\begin{aligned}
\underset{t \downarrow 0}{\limsup \left\|f-G_{t} * f\right\|_{C^{\beta}}} & \leq \limsup _{t \downarrow 0}\left\{\left\|(f-\tilde{f})-G_{t} *(f-\tilde{f})\right\|_{C^{\beta}}+\left\|\tilde{f}-G_{t} * \tilde{f}\right\|_{C^{\beta}}\right\} \\
& =\limsup _{t \downarrow 0}\left\|(f-\tilde{f})-G_{t} *(f-\tilde{f})\right\|_{C^{\beta}} \\
& \stackrel{\leq}{\leq} C\|(f-\tilde{f})\|_{C^{\beta}} .
\end{aligned}
$$

Since $h^{\beta}\left(\mathbb{R}, \mathbb{R}^{n}\right)$ is the closure of $C^{\infty}\left(\mathbb{R}, \mathbb{R}^{n}\right)$ under $\|\cdot\|_{C^{\beta}}$, this proves the statement.

Linking the heat kernel $G_{t}$ to the evolution equation $\partial_{t} u+\lambda(-\Delta)^{s / 2} u=f$ for constant $\lambda>0$ we derive the following a priori estimates.

Lemma 2.9 (Maximal regularity for constant coefficients) For all $\beta>0, \theta \in(0,1)$ with $\beta+s \theta \notin \mathbb{N}$, and $0<T<\infty, \lambda>0$ there is a constant $C=C(\beta, \theta, T, \lambda)$ such that the following holds:

Let $u \in C^{1}\left((0, T), h^{\beta}\left(\mathbb{R}, \mathbb{R}^{n}\right)\right) \cap C^{0}\left((0, T), h^{s+\beta}(\mathbb{R})\right) \cap C^{0}\left([0, T), h^{\beta+s \theta}(\mathbb{R})\right)$ such that $u(t)$ has compact support for all $t \in(0, T)$. Then

$$
\begin{aligned}
& \sup _{t \in(0, T]} t^{1-\theta}\left(\left\|\partial_{t} u\right\|_{C^{\beta}}+\|u\|_{C^{s+\beta}}\right) \\
& \leq C\left(\sup _{t \in(0, T]} t^{1-\theta}\left\|\partial_{t} u+\lambda(-\Delta)^{s / 2} u\right\|_{C^{\beta}}+\|u(0)\|_{h^{\beta+s \theta}}\right)
\end{aligned}
$$


Proof Setting $\tilde{u}(t, x):=u\left(t, \lambda^{1 / s} x\right)$ and observing that $\partial_{t} \tilde{u}(x, t)+(-\Delta)^{s / 2} \tilde{u}(x, t)=$ $\partial_{t} u\left(t, \lambda^{1 / s} x\right)+\lambda(-\Delta)^{s / 2} u\left(t, \lambda^{1 / s} x\right)$, one sees that it is enough to prove the lemma for $\lambda=1$

To this end, we first show that Duhamel's formula

$$
u(t, \cdot)=\int_{0}^{t} G_{t-\tau} * f(\tau, \cdot) d \tau+G_{t} * u(0)
$$

holds, where $f=\partial_{t} u+(-\Delta)^{s / 2} u$. For fixed $t>0$ we decompose the integral in equation (2.19) into

$$
I_{\varepsilon}:=\int_{t-\varepsilon}^{t} G_{t-\tau} * f(\tau, \cdot) d \tau
$$

and

$$
J_{\varepsilon}:=\int_{0}^{t-\varepsilon} G_{t-\tau} * f(\tau, \cdot) d \tau
$$

and see that

$$
\left\|I_{\varepsilon}\right\|_{L^{\infty}} \stackrel{\text { Lemma }}{\leq} 2.7 C \varepsilon \sup _{\tau \in(t-\varepsilon, t)}\|f(\tau, \cdot)\|_{L^{\infty}} \stackrel{\varepsilon \downarrow 0}{\longrightarrow} 0 .
$$

As our assumptions imply that $u(t) \in H^{s}\left(\mathbb{R}, \mathbb{R}^{n}\right)$, we get, comparing the Fourier transform of both sides,

$$
\left(G_{t-\tau} *\left((-\Delta)^{s / 2} u(\tau, \cdot)\right)\right)(x)=\left(\left((-\Delta)^{s / 2} G_{t-\tau}\right) * u(\tau, \cdot)\right)(x) .
$$

Partial integration in time and equation (2.20) yields

$$
\begin{aligned}
J_{\varepsilon} & =\int_{0}^{t-\varepsilon} G_{t-\tau} * \partial_{t} u(\tau, \cdot) d \tau+\int_{0}^{t-\varepsilon} G_{t-\tau} *(-\Delta)^{s / 2} u(\tau, \cdot) d \tau \\
& =G_{\varepsilon} * u(t-\varepsilon, \cdot)-G_{t} * u(0, \cdot)+\int_{0}^{t-\varepsilon}\left(\partial_{\tau}\left(G_{t-\tau}\right)+(-\Delta)^{s / 2} G_{t-\tau}\right) * u d \tau \\
& \stackrel{(2.10)}{=} G_{\varepsilon} * u(t-\varepsilon, \cdot)-G_{t} * u(0, \cdot) \stackrel{\text { Lemma } 2.8}{\longrightarrow} u(t, \cdot)-G_{t} * u(0, \cdot) .
\end{aligned}
$$

in $C^{\beta}$ as $\varepsilon \searrow 0$. This proves Equation (2.19).

From Lemma 2.7 we get

$$
\left\|G_{t} * u_{0}\right\|_{C^{s+\beta}} \leq C t^{\theta-1}\left\|u_{0}\right\|_{C^{\beta+s \theta}}
$$

We decompose $v(t):=\int_{0}^{t} G_{t-\tau} * f(\tau, \cdot) d \tau=v_{1}(t)+v_{2}(t)$ where

$$
v_{1}(t)=G_{t / 2} * v(t / 2), \quad v_{2}(t)=\int_{\tau=t / 2}^{t} G_{t-\tau} * f(\tau, \cdot) d \tau .
$$


Then the definition of $\|\cdot\|_{Y_{T}^{\theta, \beta}}$ and the estimates for the heat kernel in Lemma 2.7 lead to

$$
\left\|v_{1}(t)\right\|_{C^{s+\beta}} \leq C(t / 2)^{-1}\|f\|_{Y_{T}^{\theta, \beta}} \int_{0}^{t / 2} \tau^{\theta-1} d \tau \leq C(t / 2)^{\theta-1}\|f\|_{Y_{T}^{\theta, \beta}} .
$$

As

$$
\xi^{1-\eta} \int_{\frac{t}{2}}^{t}(t-\tau+\xi)^{-2+\eta} d \tau=\frac{\xi^{1-\eta}}{1-\eta}\left(\xi^{\eta-1}-\left(\frac{t}{2}+\xi\right)^{\eta-1}\right) \leq \frac{1}{1-\eta}
$$

for $\xi>0$ and $\eta \in(0,1)$, we get

$$
\begin{aligned}
& \left\|\xi^{1-\eta}\left(G_{\xi} * v_{2}(t)\right)\right\|_{C^{2 s+\beta-s \eta}}=\left\|\xi^{1-\eta} \int_{t / 2}^{t}\left(G_{t-\tau+\xi} * f\right) d \tau\right\|_{C^{2 s+\beta-s \eta}} \\
& \stackrel{\text { Lemma }}{\leq} C .7 \xi^{1-\eta} \int_{t / 2}^{t}(t-\tau+\xi)^{-2+\eta} \tau^{\theta-1} d \tau\|f\|_{Y_{T}^{\theta, \beta}} \\
& \leq C(t / 2)^{\theta-1}\|f\|_{Y_{T}^{\theta, \beta}}
\end{aligned}
$$

and

$$
\begin{aligned}
\left\|\xi^{1-\eta} \frac{d}{d \xi}\left(G_{\xi} * v_{2}\right)\right\|_{C^{s+\beta-s \eta}} & =\left\|\xi^{1-\eta} \int_{t / 2}^{t}\left(\partial_{t} G_{t-\tau+\xi} * f\right) d \tau\right\|_{C^{s+\beta-s \eta}} \\
& \leq C \xi^{1-\eta} \int_{t / 2}^{t}(t-\tau+\xi)^{-2+\eta} \tau^{\theta-1} d \tau\|f\|_{Y_{T}^{\theta, \beta}} \\
& \leq C(t / 2)^{\theta-1}\|f\|_{Y_{T}^{\theta, \beta}}
\end{aligned}
$$

Hence, by the estimate (2.6) for $X_{T / 2}^{\eta, s+\beta-s \eta}$

$$
\begin{aligned}
& \left\|v_{2}(t)\right\|_{C^{s+\beta}} \\
& \quad \leq C \sup _{\xi \in(0, T / 2)}\left(\xi^{1-\eta}\left\|G_{\xi} * v_{2}(t)\right\|_{C^{2 s+\beta-s \eta}}+\left\|\partial_{\xi}\left(G_{\xi} * v_{2}(t)\right)\right\|_{C^{s+\beta-s \eta}}\right) \\
& \quad \leq C t^{\theta-1}\|f\|_{Y_{T}^{\theta, \beta} .}
\end{aligned}
$$

From (2.19), (2.21), (2.22), and (2.23) we obtain the desired estimate for $\|u\|_{C^{s+\beta}}$.

The estimate for $\partial_{t} u$ then follows from $\partial_{t} u=f-(-\Delta)^{s / 2} u$ and the triangle inequality. 
Lemma 2.10 (Maximal regularity) Let $\Lambda, T>0, n \in \mathbb{N}$, and $\beta>0, \theta \in(0,1)$ with $\beta+s \theta \notin \mathbb{N}$ be given. Then there is a constant $C=C(\Lambda, \beta, \theta, n, T),<\infty$ such that the following holds: For all

$$
\begin{aligned}
& a \in C^{1}\left([0, T], h^{\beta}(\mathbb{R} / \mathbb{Z},[1 / \Lambda, \infty))\right), \\
& \quad b \in C^{0}\left((0, T), L\left(h^{\beta}\left(\mathbb{R} / \mathbb{Z}, \mathbb{R}^{n}\right), h^{\beta}\left(\mathbb{R} / \mathbb{Z}, \mathbb{R}^{n}\right)\right)\right)
\end{aligned}
$$

with

$$
\|a\|_{C^{1}\left([0, T], C^{\beta}\right)}+t^{1-\theta}\|b(t)\|_{L\left(h^{\beta}, h^{\beta}\right)} \leq \Lambda
$$

and all $u \in C^{1}\left((0, T), h^{\beta}\left(\mathbb{R} / \mathbb{Z}, \mathbb{R}^{n}\right)\right) \cap C^{0}\left((0, T), h^{s+\beta}\right) \cap C^{0}\left([0, T], h^{\beta+s \theta}\right)$ we have

$$
\|u\|_{X_{T}^{\theta, \beta}} \leq C\left(\left\|\partial_{t} u+a Q^{s-1} u+b u\right\|_{Y_{T}^{\theta, \beta}}+\|u(0)\|_{h^{\beta+s \theta}}\right)
$$

i.e.

$$
\begin{aligned}
& \sup _{t \in[0, T]} t^{1-\theta}\left\{\left\|\partial_{t} u(t)\right\|_{C^{\beta}}+\|u(t)\|_{C^{s+\beta}}\right\} \\
& \quad \leq C\left(\sup _{t \in[0, T]} t^{1-\theta}\left\|\partial_{t} u(t)+a(t) Q^{s-1} u(t)+b(t) u(t)\right\|_{C^{\beta}}+\|u(0)\|_{h^{\beta+s \theta}}\right) .
\end{aligned}
$$

Proof Note that it is enough to prove the statement for small $T$. Let us fix $T_{0}>0$ and assume that $T \leq T_{0}$. Furthermore, we use the embedding $h^{\beta}\left(\mathbb{R} / \mathbb{Z}, \mathbb{R}^{n}\right) \rightarrow h^{\beta}\left(\mathbb{R}, \mathbb{R}^{n}\right)$ and extend the definition of $Q^{s-1}$ to functions $f$ defined on $\mathbb{R}$ by setting

$$
Q^{s-1} f(x):=p . v \int_{[-1 / 2,1 / 2]}\left(2 \frac{f(x+w)-f(x)-w f^{\prime}(x)}{w^{2}}-f^{\prime \prime}(x)\right) \frac{d w}{|w|^{s-1}} .
$$

Step $1 \beta \in(0,1)$ and $b=0$

Let $\phi, \psi \in C^{\infty}(\mathbb{R})$ be two cutoff functions satisfying

$$
\begin{aligned}
\chi_{B_{1 / 2}(0)} & \leq \phi \leq \chi_{B_{1}(0)} \\
\chi_{B_{2}(0)} & \leq \psi \leq \chi_{B_{4}(0)} .
\end{aligned}
$$

and $\phi_{r}(x):=\phi(x / r), \psi_{r}(x)=\psi(x / r)$. We set

$$
f=\partial_{t} u+a Q^{s-1} u .
$$

For $r<1 / 8$ we set $a_{0}=a(0,0)$ and calculate

$$
\begin{aligned}
& \partial_{t}\left(u \phi_{r}\right)+a_{0} c_{s}\left(-\Delta_{\mathbb{R}}\right)^{s / 2}\left(u \phi_{r}\right) \\
& \quad=\left(\partial_{t} u+a Q^{s-1} u\right) \phi_{r}-a\left(Q^{s-1}(u) \phi_{r}-Q^{s-1}\left(u \phi_{r}\right)\right)
\end{aligned}
$$




$$
\begin{aligned}
& +\left(a-a_{0}\right) Q^{s-1}\left(u \phi_{r}\right)-a_{0}\left(Q^{s-1}\left(u \phi_{r}\right)-c_{s}\left(-\Delta_{\mathbb{R}}\right)^{s / 2}\left(u \phi_{r}\right)\right) \\
= & f \phi_{r}-f_{1}-f_{2}-f_{3},
\end{aligned}
$$

where

$$
\begin{aligned}
& f_{1}:=a\left(Q^{s-1}(u) \phi_{r}-Q^{s-1}\left(u \phi_{r}\right)\right) \\
& f_{2}:=\left(a-a_{0}\right) Q^{s-1}\left(u \phi_{r}\right) \\
& f_{3}:=a_{0}\left(Q^{s-1}\left(u \phi_{r}\right)-c_{s}\left(-\Delta_{\mathbb{R}}\right)^{s / 2}\left(u \phi_{r}\right)\right) .
\end{aligned}
$$

From Lemma 2.9 we get

$$
\begin{aligned}
& t^{1-\theta}\left(\left\|\partial_{t} u(t) \phi_{r}\right\|_{C^{\beta}}+\left\|u(t) \phi_{r}\right\|_{C^{s+\beta}}\right) \\
& \leq C\left(\sup _{\tau \in[0, T]} \tau^{1-\theta}\left(\left\|f(\tau) \phi_{r}\right\|_{C^{\beta}}+\left\|f_{1}(\tau)\right\|_{C^{\beta}}+\left\|f_{2}(\tau)\right\|_{C^{\beta}}+\left\|f_{3}(\tau)\right\|_{C^{\beta}}\right)\right. \\
& \left.\quad+\|u(0)\|_{C^{\beta+s \theta}}\right) \\
& \quad \leq C\left(\sup _{\tau \in[0, T]} \tau^{1-\theta}\left(\|f(\tau)\|_{C^{\beta}}+\left\|f_{1}(\tau)\right\|_{C^{\beta}}+\left\|f_{2}(\tau)\right\|_{C^{\beta}}+\left\|f_{3}(\tau)\right\|_{C^{\beta}}\right)\right. \\
& \left.\quad+\|u(0)\|_{C^{\beta+s \theta}}\right) .
\end{aligned}
$$

Using Lemma 7.1, we obtain

$$
\left\|f_{1}(\tau)\right\|_{C^{\beta}} \leq C\|u(\tau)\|_{C^{\beta+(s-1)}}\left\|\phi_{r}\right\|_{C^{s+\beta}}
$$

Using $\left|a(x, t)-a_{0}\right| \leq\left(|x|^{\beta}+T\right)$, we derive

$$
\begin{aligned}
& \left\|f_{2}\right\|_{C^{\beta}} \leq\left\|\psi_{r}\left(a-a_{0}\right) Q^{s-1}\left(u \phi_{r}\right)\right\|_{C^{\beta}}+\left\|\left(\psi_{r}-1\right)\left(a-a_{0}\right) Q^{s-1}\left(u \phi_{r}\right)\right\|_{C^{\beta}} \\
& \leq C_{1}\left((4 r)^{\beta}+T\right)\left\|u \phi_{r}\right\|_{C^{s+\beta}}+C\left\|u \psi_{r}\right\|_{C^{s}}+\left\|\left(\psi_{r}-1\right)\left(a-a_{0}\right) Q^{s-1}\left(u \phi_{r}\right)\right\|_{C^{\beta}}
\end{aligned}
$$

where $C_{1}$ does not depend on $r$ or $T$. Since $\operatorname{spt}\left(1-\psi_{r}\right) \subset \mathbb{R}-B_{2 r}(0)$ and spt $\phi_{r} \subset$ $B_{r}(0)$, we see that

$$
\begin{aligned}
& \left(\psi_{r}-1\right)\left(a-a_{0}\right) Q^{s-1}\left(u \phi_{r}\right)(x) \\
& =\left(\psi_{r}(x)-1\right)\left(a(x, t)-a_{0}\right) \int_{[-1 / 2,1 / 2]-[-r, r]} 2 \frac{u(x+w) \phi_{r}(x+w)}{w^{2}} \frac{d w}{|w|^{s-1}}
\end{aligned}
$$

and hence

$$
\left\|\left(\psi_{r}-1\right)\left(a-a_{0}\right) Q^{s-1}\left(u \phi_{r}\right)\right\|_{C^{\beta}} \leq C(\psi, \phi, r)\|u\|_{C^{\beta}} .
$$


This leads to

$$
\left\|f_{2}(\tau)\right\|_{C^{\beta}} \leq C_{1}\left((4 r)^{\beta}+T\right)\left\|u(\tau) \phi_{r}\right\|_{C^{s+\beta}}+C\|u(\tau)\|_{C^{\beta}} .
$$

Furthermore,

$$
\left\|f_{3}\right\|_{C^{\beta}} \leq C(\phi, r)\|u\|_{C^{2+\beta}}
$$

since for $v \in C^{s+\beta}(\mathbb{R})$ with compact support we have

$$
Q^{s-1}(v)-c_{s}\left(-\Delta_{\mathbb{R}}\right)^{s / 2}(v)=-\int_{\mathbb{R}-[-1 / 2,1 / 2]}\left(2 \frac{v(u+w)-v(u)}{w^{2}}-v^{\prime \prime}(u)\right) \frac{d w}{|w|^{s-1}} .
$$

and hence

$\left\|Q^{s-1}(v)-c_{S}\left(-\Delta_{\mathbb{R}}\right)^{s / 2}(v)\right\|_{C^{\beta}} \leq\left(8\|v\|_{C^{\beta}}+\|v\|_{C^{2+\beta}}\right) \cdot 2 \int_{\frac{1}{2}}^{\infty} \frac{1}{|w|^{s-1}} d w \leq C\|v\|_{C^{2+\beta}}$.

Summing up, we thus get

$$
\begin{aligned}
& \sup _{t \in(0, T]} t^{1-\theta}\left(\left\|\partial_{t}\left(u \phi_{r}\right)(t)\right\|_{C^{\beta}}+\left\|u(t) \phi_{r}\right\|_{C^{s+\beta}}\right) \\
& \leq C_{1} \Lambda\left((2 r)^{\beta}+T\right) \sup _{\tau \in(0, T]} \tau^{1-\theta}\left\|u \phi_{r}\right\|_{C^{s+\beta}} \\
& \quad+C(\phi, \psi, r, \Lambda))\left(\operatorname { s u p } _ { \tau \in ( 0 , T ] } \left(\tau^{1-\theta}\|f(\tau)\|_{C^{\beta}}\right.\right. \\
& \left.\quad+\tau^{1-\theta}\|u(\tau)\|_{C^{\beta+s-1}}+\tau^{1-\theta}\left\|u\left(\tau \|_{C^{s}}\right)+\right\| u_{0} \|_{C^{\beta+s \theta}}\right),
\end{aligned}
$$

where $C_{1}$ does not depend on $r$. Choosing $r$ and $T$ small enough and absorbing the first term on the right hand side, leads to

$$
\begin{aligned}
& \sup _{t \in(0, T]} t^{1-\theta}\left(\left\|\partial_{t} u\right\|_{C^{\beta}\left(B_{r / 2}(0)\right)}+\|u\|_{C^{s+\beta}\left(B_{r / 2}(0)\right)}\right) \\
& \quad \leq C(\phi, \psi, r, \Lambda))\left(\operatorname { s u p } _ { \tau \in ( 0 , T ] } \left(\tau^{1-\theta}\|f(\tau)\|_{C^{\beta}}+\tau^{1-\theta}\|u(\tau)\|_{C^{\beta+s-1}}\right.\right. \\
& \left.\quad+\tau^{1-\theta}\left\|u\left(\tau \|_{C^{s}}\right)+\right\| u(0) \|_{C^{\beta+s \theta}}\right) .
\end{aligned}
$$

Of course, the same inequality holds for all balls of radius $r / 4$. Thus, covering $[0,1]$ with balls of radius $r / 4$ we obtain 


$$
\begin{aligned}
& \sup _{t \in(0, T]} t^{1-\theta}\left(\left\|\partial_{t} u(t)\right\|_{C^{\beta}}+\|u(t)\|_{C^{s+\beta}}\right) \\
& \leq C\left(\operatorname { s u p } _ { t \in ( 0 , T ] } \left(t^{1-\theta}\|f(t)\|_{C^{\beta}}+t^{1-\theta}\|u(t)\|_{C^{\beta+s-1}}\right.\right. \\
& \left.\left.\quad+t^{1-\theta}\|u(t)\|_{C^{s}}\right)+\|u(0)\|_{C^{\beta+s \theta}}\right)
\end{aligned}
$$

Using the interpolation inequality for Hölder spaces

$$
\|u\|_{C^{\beta+s-1}} \leq \varepsilon\|u\|_{C^{s+\beta}}+C(\varepsilon)\|u\|_{C^{\beta}}
$$

and absorbing, this leads to

$$
\begin{aligned}
& \sup _{t \in(0, T]} t^{1-\theta}\left(\left\|\partial_{t} u(t)\right\|_{C^{\beta}}+\|u(t)\|_{C^{s+\beta}}\right) \\
& \quad \leq C\left(\sup _{t \in(0, T]}\left(t^{1-\theta}\|f(t)\|_{C^{\beta}}+t^{1-\theta}\|u(t)\|_{C^{\beta}}\right)+\|u(0)\|_{C^{\beta+s} \theta}\right) .
\end{aligned}
$$

Since

$$
\begin{aligned}
\|u(t)\|_{C^{\beta}} & \leq \int_{0}^{t}\left\|\partial_{t} u(\tau)\right\|_{C^{\beta}} d \tau+\|u(0)\|_{C^{\beta+s \theta}} \\
& \leq \int_{0}^{T} \tau^{\theta-1} d \tau \sup _{\tau \in(0, T]} \tau^{1-\theta}\left\|\partial_{t} u(\tau)\right\|_{C^{\beta}}+\|u(0)\|_{C^{\beta+s \theta}} \\
& \leq \frac{1}{\theta} T^{\theta} \sup _{\tau \in(0, T]} \tau^{1-\theta}\left\|\partial_{t} u(\tau)\right\|_{C^{\beta}}+\|u(0)\|_{C^{\beta+s \theta}},
\end{aligned}
$$

we can absorb the first term for $T>0$ small enough to obtain

$$
\begin{aligned}
& \sup _{t \in(0, T]} t^{1-\theta}\left(\left\|\partial_{t} u(t)\right\|_{C^{\beta}}+\|u(t)\|_{C^{s+\beta}}\right) \\
& \quad \leq C\left(\left(\sup _{t \in(0, T]} t^{1-\theta}\|f(t)\|_{C^{\beta}}\right)+\|u(0)\|_{C^{\beta+s \theta}}\right) .
\end{aligned}
$$

Step 2 General $\beta$ but $b=0$

Let $k \in \mathbb{N}_{0}, \tilde{\beta} \in(0,1)$ and let the lemma be true for $\beta=k+\tilde{\beta}$. We deduce the statement for $\beta=k+1+\tilde{\beta}$.

From $\partial_{t} u+a Q^{s-1} u=f$ we deduce that

$$
\partial_{t}\left(\partial_{x} u\right)+a Q^{s-1} \partial_{x} u=\partial_{x} f-\left(\partial_{x} a\right) Q^{s-1} u
$$


and we obtain by applying the induction hypothesis to get

$$
\begin{aligned}
\left\|\partial_{x} u\right\|_{X_{T}^{\theta, k+\tilde{\beta}}} & \leq C\left(\left\|\partial_{x} f\right\|_{Y_{T}^{\theta, k+\tilde{\beta}}}+\left\|\left(\partial_{x} a\right) Q^{s-1} u\right\|_{Y_{T}^{\theta, k+\tilde{\beta}}}+\left\|\partial_{x} u(0)\right\|_{C^{\beta+s \theta}}\right) \\
& \leq C\left(\|f\|_{Y_{T}^{\theta, k+\tilde{\beta}}}+\|u\|_{X_{T}^{\theta, k+\tilde{\beta}}}+\left\|\partial_{x} u(0)\right\|_{C^{\beta+s \theta}}\right) \\
& \leq C\left(\|f\|_{Y_{T}^{\theta, k+\tilde{\beta}}}+\left\|\partial_{x} u(0)\right\|_{C^{\beta+s \theta}}\right) .
\end{aligned}
$$

\section{Step 3 General $\beta$ and $b$}

From Step 2 we get

$$
\|u\|_{X_{T}^{\theta, \beta}} \leq C\left(\|f\|_{Y_{T}^{\theta, \beta}}+\|((t, x) \mapsto b(t)(u(t))(x))\|_{Y_{T}^{\theta, \beta}}+\left\|u_{0}\right\|_{C^{\beta+s \theta}}\right) .
$$

As

$$
\|((t, x) \mapsto b(t)(u(t))(x))\|_{Y_{T}^{\theta, \beta}}=\sup _{\tau \in(0, T]} \tau^{1-\theta}\|b(\tau)(u(\tau))\|_{C^{\beta}} \leq C \sup _{\tau \in(0, T]}\|u(\tau)\|_{C^{\beta}}
$$

and by (2.24)

$$
\|u(t)\|_{C^{\beta}} \leq \frac{1}{\theta} T^{\theta} \sup _{\tau \in(0, T]} \tau^{1-\theta}\left\|\partial_{t} u(\tau)\right\|_{C^{\beta}}+\|u(0)\|_{C^{\beta+s \theta}} .
$$

we get, absorbing the first term for $T>0$ small enough,

$$
\|u\|_{X_{T}^{\theta, \beta}} \leq C\left(\|f\|_{Y_{T}^{\theta, \beta}}+\left\|u_{0}\right\|_{C^{\beta+s \theta}}\right) .
$$

Now we can finally prove Theorem 2.6.

Proof of Theorem 2.6 It only remains to show that this mapping $J$ is onto. To prove this, we use the method of continuity for the family of operators $J_{\tau}: u \mapsto\left(u(0), \partial_{t} u+\right.$ $\left((1-\tau) \lambda Q^{s-1} u+\tau\left(a Q^{s-1} u+b u\right)\right)$. In view of Lemma 5.2 in [15], we have to show is that $J_{0}$ is onto.

By [17, Lemma 2.3] and [24, Proposition 1.4] we have for all $f \in H^{s}\left(\mathbb{R} / \mathbb{Z}, \mathbb{R}^{n}\right)$

$$
Q^{s-1}(f)^{\wedge}(k)=\lambda_{k}|2 \pi k|^{s} \hat{f}(k)
$$

where

$$
\lambda_{k}=c_{s}+O\left(\frac{1}{k}\right)
$$


for some positive constants $c_{s}$. For $u_{0}, f$ in $C^{\infty}$ a smooth solution of the equation

$$
\left\{\begin{array}{l}
\partial_{t} u+\lambda Q^{s-1} u=f \quad \forall t \in(0, T] \\
u(0)=u_{0}
\end{array}\right.
$$

can be given by Duhamel's formula

$$
u(t, x)=\sum_{k \in \mathbb{Z}} \hat{u}_{0}(k) e^{-t \lambda \lambda_{k}|2 \pi k|^{s}} e^{2 \pi i k x}+\int_{0}^{t} \sum_{k \in \mathbb{Z}}(f(\tau))^{\wedge}(k) e^{-(t-\tau) \lambda \lambda_{k}|2 \pi k|^{s}} d \tau .
$$

This formula can easily be checked comparing the Fourier coefficients.

Let now $u_{0} \in h^{\beta+s \theta}\left(\mathbb{R} / \mathbb{Z}, \mathbb{R}^{n}\right)$ and $f \in Y_{T}^{\theta, \beta}$. We set $f_{k}(t):=f(t+1 / k)$ and observe that

$$
f_{k} \rightarrow f \quad \text { in } C^{0}\left((0, T-\varepsilon], h^{\beta}\left(\mathbb{R} / \mathbb{Z}, \mathbb{R}^{n}\right)\right)
$$

for all $\varepsilon>0$. Since $f_{k} \in C^{0}\left([0, T-1 / k], h^{\beta}\left(\mathbb{R} / \mathbb{Z}, \mathbb{R}^{n}\right)\right)$, we can find functions $f_{n, k} \in C^{\infty}\left([0, T-1 / k] \times \mathbb{R} / \mathbb{Z}, \mathbb{R}^{n}\right)$ such that $f_{n, k} \rightarrow f_{k}$ in $C^{0}\left([0, T-1 / k], C^{\beta}\right)$ for $n \rightarrow \infty$ and smooth $u_{0}^{(n)}$ converging to $u_{0}$ in $h^{\beta+s \theta}$. Let $u_{n, k} \in C^{\infty}$ be the solution of

$$
\left\{\begin{array}{l}
\partial_{t} u_{n, k}+Q^{s-1} u_{n, k}=f_{n, k} \\
u_{n, k}(0)=u_{0}^{(n)}
\end{array}\right.
$$

Using the a priori estimate of Lemma 2.10, one deduces that the sequence $\left\{u_{n, k}\right\}_{n \in \mathbb{N}}$ is a Cauchy sequence in $X_{T-\varepsilon}^{\theta, \beta}$ for every $\varepsilon>0$. The limit $u_{n}$ solves the equation

$$
\left\{\begin{array}{l}
\partial_{t} u_{k}+Q^{s-1} u_{k}=f_{k} \\
u_{k}(0)=u_{0}
\end{array}\right.
$$

Using the a priori estimates again, one sees that $\left\{u_{k}\right\}_{k \in \mathbb{N}}$ is bounded in $X_{T-\varepsilon}^{\theta, \beta}$. Since $X_{T-\varepsilon}^{\theta, \beta}$ is embedded continuously in $C^{\theta / 2}\left([0, T-\varepsilon], h^{\beta+s \frac{\theta}{2}}\right)$ and $C^{1-\eta}([\delta, T-$ $\left.\varepsilon], h^{\beta+s \eta}\right)$ for all $\eta \in(0,1), \varepsilon, \delta>0$, we can assume, after going to a subsequence, that there is a $u_{\infty} \in X_{T}^{\theta, \beta}$ such that

$$
u_{k} \rightarrow u_{\infty} \text { in } C^{0}\left((0, T-\varepsilon), C^{s+\tilde{\beta}}\right)
$$

for $0 \leq \tilde{\beta}<\beta, \varepsilon>0$ and

$$
u_{\infty}(0)=u_{0}
$$

Hence we get

$$
\partial_{t} u_{k}=f_{k}-\lambda Q^{s-1} u_{k} \rightarrow f+\lambda Q^{s-1} u_{\infty} \quad \text { in } C^{0}\left((0, T-\varepsilon), C^{s+\tilde{\beta}}\right)
$$


for all $\varepsilon>0$ which implies that $u_{\infty}$ solves

$$
\left\{\begin{array}{l}
\partial_{t} u_{\infty}+\lambda Q^{s-1} u_{\infty}=f \\
u_{\infty}(0)=u_{0}
\end{array}\right.
$$

\subsubsection{The quasilinear equation}

Now we are in position to prove short time existence for quasilinear equations and $C^{1}$-dependence on the initial data.

Proposition 2.11 (Short time existence) Let $0<\beta, 0<\theta<\sigma<1, \beta, \beta+s \theta, \beta+$ $s \sigma \notin \mathbb{N}_{0}, U \subset C^{\beta+s \theta}\left(\mathbb{R} / \mathbb{Z}, \mathbb{R}^{n}\right)$ be open and let $a \in C^{1}\left(U, C^{\beta}(\mathbb{R} / \mathbb{Z},(0, \infty))\right)$, $f \in C^{1}\left(U, C^{\beta}\left(\mathbb{R} / \mathbb{Z}, \mathbb{R}^{n}\right)\right)$.

Then for every $u_{0} \in h^{\beta+s \sigma}\left(\mathbb{R} / \mathbb{Z}, \mathbb{R}^{n}\right) \cap U$ there is a constant $T>0$ and a unique $u \in C^{0}\left([0, T), h^{\beta+s \sigma}\left(\mathbb{R} / \mathbb{Z}, \mathbb{R}^{n}\right)\right) \cap C^{1}\left((0, T), h^{s+\beta}\left(\mathbb{R} / \mathbb{Z}, \mathbb{R}^{n}\right)\right)$ such that

$$
\left\{\begin{array}{l}
\partial_{t} u+a(u) Q^{s-1}(u)=f(u) \\
u(0)=u_{0} .
\end{array}\right.
$$

Proof Let us first prove the existence. We set $\tilde{X}_{T}^{\sigma, \beta}:=\left\{w \in X_{T}^{\sigma, \beta}: w(0)=u_{0}\right\}$. For $w \in \tilde{X}_{T}^{\sigma, \beta}\left(\mathbb{R} / \mathbb{Z}, \mathbb{R}^{n}\right)$ let $\Phi w$ denote the solution $u$ of the problem

$$
\left\{\begin{array}{l}
\partial_{t} u+A_{0} u=B(w) w+f(w) \\
u(0)=u_{0}
\end{array}\right.
$$

where $A_{0}=a\left(u_{0}\right) Q^{s-1}$ and $B(w)=\left(a\left(u_{0}\right)-a(w)\right) Q^{s-1}$.

Let $v$ be the solution of

$$
\left\{\begin{array}{l}
\partial_{t} v+A_{0} v=f\left(u_{0}\right) \\
v(0)=u_{0}
\end{array}\right.
$$

and $\mathcal{B}_{r}(v):=\left\{w \in \tilde{X}_{T}^{\sigma, \beta}:\|w-v\|_{X_{T}^{\sigma, \beta}} \leq r\right\}$. We will show that $\Phi$ defines a contraction on $\mathcal{B}_{r}(v)$ if $r, T>0$ are small enough.

Since $a \in C^{1}\left(U, h^{\beta}\left(\mathbb{R} / \mathbb{Z}, \mathbb{R}^{n}\right)\right)$, we get

$$
\|B(z)-B(u)\|_{L\left(C^{\beta+s}, C^{\beta}\right)} \leq\|a(z)-a(u)\|_{C^{\beta}} \leq C\|z-u\|_{C^{\beta+s \theta}}
$$

for all $z, u \in C^{\beta+s \theta}$ close to $u_{0}$.

Let $w_{1}, w_{2} \in \mathcal{B}_{r}(v), r \leq 1$. Using that the space $X_{T}^{\sigma, \beta}$ is embedded continuously in $C^{\sigma-\theta}\left([0, T], h^{\beta+s \theta}\left(\mathbb{R} / \mathbb{Z}, \mathbb{R}^{n}\right)\right)$ and $w_{1}(0)=w_{2}(0)=v(0)=u_{0}$ we get

$$
\begin{aligned}
\left\|w_{2}(t)-u_{0}\right\|_{C^{\beta+s \theta}} & \leq C t^{\sigma-\theta}\left\|w_{2}\right\|_{X_{T}^{\sigma, \beta}} \leq C t^{\sigma-\theta}\left(\|v\|_{X_{T}^{\sigma, \beta}}+r\right), \\
\left\|w_{1}(t)-w_{2}(t)\right\|_{C^{\beta+s \theta}} & \leq C t^{\sigma-\theta}\left\|w_{1}-w_{2}\right\|_{X_{T}^{\sigma, \beta}} .
\end{aligned}
$$


Using Lemma 2.10, we estimate

$$
\left\|\Phi w_{1}-\Phi w_{2}\right\|_{X_{T}^{\sigma, \beta}} \leq C\left\|B\left(w_{1}\right) w_{1}-B\left(w_{2}\right) w_{2}\right\|_{Y_{T}^{\sigma, \beta}}+C\left\|f w_{1}-f w_{2}\right\|_{Y_{T}^{\sigma, \beta}}
$$

As

$$
\begin{aligned}
& t^{1-\sigma}\left\|f\left(w_{1}(t)\right)-f\left(w_{2}(t)\right)\right\|_{C^{\beta}} \leq C t^{1-\sigma}\left\|w_{1}(t)-w_{2}(t)\right\|_{C^{\beta+s \theta}} \\
& \stackrel{(2.26)}{\leq} C T^{1-\theta}\left\|w_{1}-w_{2}\right\|_{X_{T}^{\sigma, \beta}}
\end{aligned}
$$

and

$$
\begin{aligned}
& \left\|B\left(w_{1}\right) w_{1}-B\left(w_{2}\right) w_{2}\right\|_{Y_{T}^{\sigma, \beta}} \\
& \leq\left\|\left(B\left(w_{1}\right)-B\left(w_{2}\right)\right) w_{1}\right\|_{Y_{T}^{\sigma, \beta}}+\left\|B\left(w_{2}\right)\left(w_{1}-w_{2}\right)\right\|_{Y_{T}^{\sigma, \beta}} \\
& \leq C \sup _{t \in(0, T]} t^{1-\sigma}\left(\left\|w_{1}(t)-w_{2}(t)\right\|_{C^{\beta+s} \theta}\left\|w_{1}(t)\right\|_{C^{s+\beta}}\right. \\
& \left.+\left\|w_{2}(t)-u_{0}\right\|_{C^{\beta+s \theta}}\left\|w_{1}(t)-w_{2}(t)\right\|_{C^{s+\beta}}\right) \\
& \text { (2.26)\&(2.25) } \\
& \stackrel{\&(2.25)}{\leq} C \sup _{t \in(0, T]}\left(t^{\sigma-\theta}\left\|w_{1}-w_{2}\right\|_{X_{T}^{\sigma, \beta}}\left\|w_{1}\right\|_{X_{T}^{\sigma, \beta}}\right. \\
& \left.+t^{\sigma-\theta}\left(\|v\|_{X_{T}^{\sigma, \beta}}+r\right) t^{1-\sigma}\left\|w_{1}(t)-w_{2}(t)\right\|_{C^{s+\beta}}\right) \\
& \leq C\left(T^{\sigma-\theta}\left(\|v\|_{X_{T}^{\sigma, \beta}}+r\right)\left\|w_{1}-w_{2}\right\|_{X_{T}^{\sigma, \beta}}\right.
\end{aligned}
$$

we get the estimate

$$
\left\|\Phi\left(w_{1}\right)-\Phi\left(w_{2}\right)\right\|_{X_{T}^{\sigma, \beta}} \leq C\left(T^{1-\sigma}+T^{\sigma-\theta}\left(\|v\|_{X_{T}^{\sigma, \beta}}+r\right)\right)\left\|w_{1}-w_{2}\right\|_{X_{T}^{\sigma, \beta}} .
$$

Hence, $\Phi$ is a contraction on $\mathcal{B}_{r}(v)$ if $T$ and $r$ are small enough.

Similarly, we deduce from

$$
\left\{\begin{array}{l}
\partial_{t}(\Phi(w)-v)+A_{0}((\Phi(w)-v))=B(w) w+f(w)-f\left(u_{0}\right) \\
\Phi(w)(0)-v(0)=0
\end{array}\right.
$$

using Lemma 2.10 that

$$
\begin{aligned}
& \|\Phi(w)-v\|_{X_{T}^{\sigma, \beta}} \leq C\|B(w) w\|_{Y_{T}^{\sigma, \beta}}+\left\|f(w)-f\left(u_{0}\right)\right\|_{Y_{T}^{\sigma, \beta}} \\
& \leq C T^{\sigma-\theta}\|w\|_{X_{T}^{\sigma, \beta}}\|w-v\|_{X_{T}^{\beta, \theta}}+C T^{1-\theta}\|w-v\|_{X_{T}^{\sigma, \beta}}+C T^{1-\sigma}\left\|v-u_{0}\right\|_{C^{\beta+s \sigma}} \\
& <\frac{1}{2}\|w-v\|_{X_{T}^{\sigma, \beta}}+C T^{1-\theta}+C T^{1-\sigma}<r
\end{aligned}
$$

if $T$ and $r$ are small enough. Then $\phi\left(\mathcal{B}_{r}(v)\right) \subset \mathcal{B}_{r}(v)$. Hence, by Banach's fixed-point theorem there is a unique $u \in B_{r}(v)$ with $\partial_{t} u+a(u) Q^{s-1}(u) u=f(u)$. 
For the uniqueness statement, we only have to guarantee that every solution is in $Y_{T}^{\sigma, \beta}$. But this follows from Lemma 2.10.

Proposition 2.12 (Dependence on the data) Let $a, b$ be as in Proposition 2.11 and $u \in Y_{T}^{\theta, \beta}$ be a solution of the quasilinear equation

$$
\left\{\begin{array}{l}
\partial_{t} u+a(u) Q^{s-1}(u)=0 \\
u(0)=u_{0}
\end{array}\right.
$$

Then there is a neighborhood $U$ of $u_{0}$ in $h^{\beta+s \theta}$ such that for all $x \in U$ there is a solution $u_{x}$ of

$$
\left\{\begin{array}{l}
\partial_{t} u+a(u) Q^{s-1} u=0 \\
u(0)=x
\end{array}\right.
$$

Furthermore, the mapping

$$
\begin{aligned}
U & \rightarrow Y_{T}^{\theta, \beta} \\
x & \mapsto u_{x}
\end{aligned}
$$

is $C^{1}$.

Proof We define $\phi: h^{\beta+s \theta}\left(\mathbb{R} / \mathbb{Z}, \mathbb{R}^{n}\right) \times X_{T}^{\theta, \beta} \rightarrow Y_{T}^{\theta, \beta}$ by

$$
\phi(x, u):=\left(u(0)-x, \partial_{t} u+a(u) Q^{s-1} u\right)
$$

Then the Fréchet derivative of $\phi$ with respect to $u$ reads as

$$
\frac{\partial \phi(x, u)}{\partial u}(h)=\left(h(0), \partial_{t} h+a(u) Q^{s-1} h+a^{\prime}(u) h Q^{s-1} u\right) .
$$

Setting $a(t)=a(u(t))$ and $b(t)(h)=a^{\prime}(u) h Q^{s-1} u$, Theorem 2.6 tells us that this is an isomorphism between $X_{T}^{\theta, \beta}$ and $h^{\beta+s \theta} \times Y_{T}^{\theta, \beta}$. Hence, the statement of the lemma follows from the implicit function theorem on Banach spaces.

\subsubsection{Proof of Theorem 2.5}

Since the normal bundle of a curve is trivial, we can find smooth normal vector fields $v_{1}, \ldots, v_{n-1} \in C^{\infty}\left(\mathbb{R} / \mathbb{Z}, \mathbb{R}^{n}\right)$ such that for each of $x \in \mathbb{R} / \mathbb{Z}$ the vectors $v_{1}(x), \ldots, v_{n-1}(x)$ form an orthonormal basis of the space of all normal vectors to $c_{0}$ at $x$. Let $\tilde{\mathcal{V}}_{r, \beta}\left(c_{0}\right):=\left\{\left(\phi_{1}, \ldots, \phi_{n-1}\right) \in h^{\beta}\left(\mathbb{R} / \mathbb{Z}, \mathbb{R}^{n-1}\right): \sum_{i=1}^{n-1} \phi_{i} v_{i} \in \mathcal{V}_{r, \beta}\left(c_{0}\right)\right\}$.

If we have $N_{t}=\sum_{i=1}^{n-1} \phi_{i, t} \nu_{i},\left(\phi_{1, t}, \ldots \phi_{n-1, t}\right) \in \tilde{\mathcal{V}}_{r, \beta}(c)$, then (2.5), using Theorem 2.3 , can be written as 


$$
\begin{aligned}
\sum_{i=1}^{n-1}\left(\partial_{t} \phi_{i, t}\right)\left(P_{c^{\prime}(u)}^{\perp} v_{i}\right)= & -\frac{2}{\left|c^{\prime}\right|^{s}} P_{c^{\prime}}^{\perp}\left(Q^{\alpha}\left(c_{0}+\sum_{i=1}^{n-1} \phi_{i, t} \nu_{i}\right)\right) \\
& -F\left(c_{0}+\sum_{i=1}^{n-1} \phi_{i, t} v_{i}\right)+\lambda \kappa \\
= & -\frac{2}{\left|c^{\prime}\right|^{s}} \sum_{i=1}^{n-1}\left(Q^{\alpha} \phi_{i, t}\right) P_{c^{\prime}}^{\perp} v_{i} \\
& -\frac{2}{\left|c^{\prime}\right|^{S}} P_{c^{\prime}}^{\perp}\left(\sum_{i=1}^{n-1} Q^{\alpha}\left(\phi_{i, t} v_{i}\right)-\sum_{i=1}^{n-1}\left(Q^{\alpha} \phi_{i, t}\right) v_{i}+Q^{\alpha} c_{0}\right) \\
& -F\left(c_{0}+\sum_{i=1}^{n-1} \phi_{i, t} v_{i}\right)+\lambda \kappa \\
= & -\frac{2}{\left|c^{\prime}\right|^{s}} \sum_{i=1}^{n-1}\left(Q^{\alpha} \phi_{i, t}\right) P_{c^{\prime}}^{\perp} v_{i}+\tilde{F}_{c_{0}}\left(\phi_{t}\right)+\lambda \kappa
\end{aligned}
$$

where

$$
\begin{aligned}
\tilde{F}_{c_{0}}\left(\phi_{t}\right)= & -F\left(c_{0}+\sum_{i=1}^{n-1} \phi_{i, t} v_{i}\right)-\frac{2}{\left|c^{\prime}\right|^{S}} P_{c^{\prime}}^{\perp}\left(Q^{\alpha} c_{0}\right) \\
& +\frac{2}{\left|c^{\prime}\right|^{s}}\left(\sum_{i=1}^{n-1}\left(Q^{\alpha} \phi_{i, t}\right) P_{c^{\prime}}^{\perp} v_{i}-P_{c^{\prime}}^{\perp}\left(\sum_{i=1}^{n-1} Q^{\alpha} \phi_{i, t} v_{i}\right)\right) .
\end{aligned}
$$

Using Lemma 7.1 we see that $\tilde{F} \in C^{w}\left(C^{2+\beta}, C^{\beta}\right)$ for all $\beta>0$ and hence especially $\tilde{F} \in C^{w}\left(C^{\alpha+\beta}, C^{\beta}\right)$ for all $\beta>0$. Furthermore, the term $\lambda \kappa$ belongs to $C^{\omega}\left(C^{\alpha+\beta}, C^{\beta}\right)$. Since (2.3) implies that $\left\{P_{c^{\prime}}^{\perp} v_{r}: r=1, \ldots, n-1\right\}$ is a basis of the normal space of the curve $c$ at the point $u$, the mapping $A: \mathbb{R}^{n-1} \rightarrow\left(\mathbb{R} c^{\prime}(u)\right)^{\perp}$, $\left(x_{1}, \ldots, x_{n-1}\right) \mapsto \sum_{i=1}^{n-1} x_{i} P_{c^{\prime}(u)}^{\perp} v_{i}$ is invertible as long as $\left\|c^{\prime}-c_{0}^{\prime}\right\|_{L^{\infty}}<1$.

So we have brought the evolution equation into the form

$$
\partial_{t} \phi_{t}=-\frac{2}{\left|c^{\prime}\right|^{s}} Q^{\alpha} \phi_{t}+A^{-1}\left(\tilde{F}\left(\phi_{t}\right)+\lambda \kappa\right)
$$

where $A^{-1}\left(\tilde{F}\left(\phi_{t}\right)+\lambda \kappa\right) \in C^{\omega}\left(h^{\alpha+\beta}, h^{\beta}\right)$ for all $\beta>0$. Now the statement follows from Proposition 2.11, Proposition 2.12, and a standard bootstrapping argument.

\subsubsection{Proof of Theorem 2.1}

From Theorem 2.5 we get a smooth solution of

$$
\left(\partial_{t} c_{t}\right)^{\perp}=-\mathfrak{V}^{\alpha}\left(c_{t}\right)+\lambda \kappa_{c_{t}} .
$$


Let $\phi_{t}(x)$ for $(x, t) \in \mathbb{R} / \mathbb{Z} \times[0, T)$ denote the solution of

$$
\partial_{t} \phi_{t}(x)=\frac{\left.-\left\langle\partial_{t} c_{t}\left(\phi_{t}(x)\right)\right), c_{t}^{\prime}\left(\phi_{t}(x)\right)\right\rangle}{\left|c_{t}^{\prime}\left(\phi_{t}(x)\right)\right|^{2}} .
$$

We calculate for $\tilde{c}_{t}=c_{t} \circ \phi_{t}$

$$
\partial_{t}\left(\tilde{c}_{t}\right)=\left(\partial_{t} c_{t}\right)^{\perp} \circ \phi_{t}=-\mathfrak{V}^{\alpha} \tilde{c}_{t}+\lambda \kappa_{\tilde{c}_{t}} .
$$

\subsubsection{Proof of Theorem 2.2}

The proof of Theorem 2.2 is an immediate consequence of Theorem 2.5 and the following approximation argument

Lemma 2.13 Letr $: h_{i, r}^{2+\beta}\left(\mathbb{R} / \mathbb{Z}, \mathbb{R}^{n}\right) \rightarrow(0, \infty)$ be a lower semi-continuous function. Then for every camma $\in h_{i, r}^{2+\beta}\left(\mathbb{R} / \mathbb{Z}, \mathbb{R}^{n}\right)$ there is a $\gamma \in C_{i, r}^{\infty}\left(\mathbb{R} / \mathbb{Z}, \mathbb{R}^{n}\right), N \in \mathcal{V}_{r}(\gamma)$ and a diffeomorphism $\psi \in C^{2+\beta}(\mathbb{R} / \mathbb{Z}, \mathbb{R} / \mathbb{Z})$ such that camma $\%=\gamma+N$.

Proof of Lemma 2.13 Let $\tilde{c} \in h_{i, r}^{2+\beta}\left(\mathbb{R} / \mathbb{Z}, \mathbb{R}^{n}\right)$ and let us set $c_{0}=c$ and $c_{\varepsilon}:=\phi_{\varepsilon} * \tilde{c}$ where $\phi_{\varepsilon}(x)=\varepsilon^{-1} \phi(x / \varepsilon)$ is a smooth smoothing kernel. Since $h_{i, r}^{2+\beta}\left(\mathbb{R} / \mathbb{Z}, \mathbb{R}^{n}\right)$ is an open subset of $h^{2+\beta}\left(\mathbb{R} / \mathbb{Z}, \mathbb{R}^{n}\right)$ and $\left(\varepsilon \rightarrow c_{\varepsilon}\right) \in C^{0}\left([0, \infty), h^{2+\beta}\left(\mathbb{R} / \mathbb{Z}, \mathbb{R}^{n}\right)\right)$, we get $c_{\varepsilon} \in h_{i, r}^{2+\beta}\left(\mathbb{R} / \mathbb{Z}, \mathbb{R}^{n}\right)$ for $\varepsilon$ small enough.

Since $c_{\varepsilon} \in C^{0}\left([0, \infty), h^{2+\beta}\left(\mathbb{R} / \mathbb{Z}, \mathbb{R}^{n}\right)\right)$ there is an open neighborhood $U$ of the set $c(\mathbb{R} / \mathbb{Z})$ and an $\varepsilon_{0}>0$ such that the nearest neighborhood retract $r_{\varepsilon}: U \rightarrow \mathbb{R} / \mathbb{Z}$ onto $c_{\varepsilon}$ is defined on $U$ simultaneously for all $0 \leq \varepsilon<\varepsilon_{0}$. Note, that these retracts $r_{\varepsilon}$ are smooth as the curves $c_{\varepsilon}$ are smooth and

$$
\begin{aligned}
{\left[0, \varepsilon_{0}\right) \times U } & \rightarrow \mathbb{R} / \mathbb{Z} \\
(\varepsilon, x) & \mapsto r_{\varepsilon}(x)
\end{aligned}
$$

belongs to $C^{0}\left(\left[0, \varepsilon_{0}\right), C^{1+\beta}\right)$.

We set $\psi_{\varepsilon}(x):=r_{\varepsilon}(c(x))$. Now $\psi_{0}=i d_{\mathbb{R} / \mathbb{Z}}, \psi_{\varepsilon}$ is a $C^{1+\beta}$ diffeomorphism for $\varepsilon>0$ small enough since the subset of diffeomorphism is open in $C^{1+\beta}$. Hence, we can set $N_{\varepsilon}(x)=c_{\varepsilon}\left(\psi_{\varepsilon}^{-1}(x)\right)-c_{0}(x)$ for $\varepsilon_{0}$ small enough. We will show that $c:=c_{\varepsilon}$, $N:=N_{\varepsilon}$, and $\psi:=\psi_{\varepsilon}$ satisfy the statement of the lemma if $\varepsilon$ is small enough.

From $\psi_{\varepsilon}(x):=r_{\varepsilon}(c(x))$ we deduce that $\psi_{\varepsilon}$ is in fact a $C^{2+\beta}$ diffeomorphism, as $r_{\varepsilon}$ is smooth.

Since

$$
N_{\varepsilon}=c_{\varepsilon} \circ \psi_{\varepsilon}^{-1}-c_{0} \in C^{0}\left([0, \infty), C^{1+\beta}\left(\mathbb{R} / \mathbb{Z}, \mathbb{R}^{n}\right)\right)
$$

and $N_{0}=0$, we furthermore get

$$
\left\|N_{\varepsilon}\right\|_{C^{1}} \stackrel{\varepsilon \searrow 0}{\longrightarrow} 0 .
$$


Since $r$ is lower semi-continuous and $r\left(c_{0}\right)>0$, we hence get $\left\|N_{\varepsilon}\right\|_{C^{1}}<r\left(c_{\varepsilon}\right)$ for small $\varepsilon$. As $N_{\varepsilon} \in h^{2+\beta}$ we deduce that $N_{\varepsilon} \in \mathcal{V}_{r}\left(c_{\varepsilon}\right)$ if $\varepsilon>0$ is small enough.

Proof Let $\tilde{c}_{0} \in h_{i, r}^{\beta}(\mathbb{R} / \mathbb{Z})$. By Lemma 2.13 there is a curve $c_{0} \in C_{i, r}^{\infty}$, an $N_{0} \in \mathcal{V}_{r, \beta}(c)$ and a diffeomorphism $\psi \in C^{\beta}$ such that

$$
\tilde{c}_{0} \circ \psi=c_{0}+N_{0} .
$$

Theorem 2.5 tells us that there is a solution $c_{t}=c_{0}+N_{N_{0}, t}$ of

$$
\left(\partial_{t} c_{t}\right)^{\perp}=-\mathfrak{V}^{\alpha}\left(c_{t}\right)+\lambda \kappa_{c_{t}} .
$$

The family of curves $c_{t} \circ \psi^{-1}$ is a solution we were looking for.

If on the other hand $\tilde{c}_{t}$ is a solution as in the theorem for the initial data $\tilde{c}_{0}$, then for some time there is a $N_{t}$ and a smooth family of reparametrizations $\tilde{\psi}_{t}$ such that $c_{t} \circ \tilde{\psi}_{t}=\tilde{c}_{0}+N_{t}$ and

$$
\partial_{t}^{\perp}\left(c_{0}+N_{t}\right)=-\mathfrak{V}^{\alpha}\left(c_{0}+N_{t}\right)+\lambda \kappa_{c_{0}+N_{t}}
$$

The uniqueness now follows from the uniqueness of the solution to the above equation in Theorem 2.5.

\section{Long time existence}

The aim of this section is to prove the following long time existence result.

Theorem 3.1 Let $c_{0} \in C^{\infty}\left(\mathbb{R} / \mathbb{Z}, \mathbb{R}^{n}\right)$. Then there exists a unique solution $c \in$ $C^{0}\left([0, \infty), C^{\infty}\left(\mathbb{R} / \mathbb{Z}, \mathbb{R}^{n}\right)\right) \cap C^{\infty}\left((0, \infty), C^{\infty}\left(\mathbb{R} / \mathbb{Z}, \mathbb{R}^{n}\right)\right)$ to $(1.4)$ with initial data $c(0)=c_{0}$. This solution subconverges, after suitable re-parameterizations and translations, to a smooth critical point of $E^{\alpha}+\lambda L$.

Let me first sketch the strategy of the proof. Since we are looking at negative gradient flows of $E^{\alpha}+\lambda L$ we have for a solution $c(t)$ of Eq. (1.4)

$$
E^{\alpha}(c(t))+\lambda L(c(t)) \leq E^{\alpha}(c(0))+\lambda L(c(0))
$$

for all $t \in[0, T)$. So both, the energy and the length of the curve, is uniformly bounded in time. As Abrams et al. [1] have shown that

$$
E^{\alpha}(c) \geq E^{\alpha}\left(\mathbb{S}^{1}\right)=m_{\alpha}>0
$$

for all closed curves $c$ of unit length, we get from the scaling of the energy

$$
E^{\alpha}(c)=L^{2-\alpha} E^{\alpha}\left(\frac{c}{L(c)}\right) \geq L^{2-\alpha} m_{\alpha}
$$


and thus

$$
\begin{aligned}
L\left(c_{t}\right) & \geq\left(\frac{m_{\alpha}}{E^{\alpha}\left(c_{t}\right)}\right)^{\frac{1}{\alpha-2}} \geq\left(\frac{m_{\alpha}}{E^{\alpha}\left(c_{t}\right)+\lambda L\left(c_{t}\right)}\right)^{\frac{1}{\alpha-2}} \\
& \geq\left(\frac{m_{\alpha}}{E^{\alpha}\left(c_{0}\right)+\lambda L\left(c_{0}\right)}\right)^{\frac{1}{\alpha-2}}>0
\end{aligned}
$$

uniformly in $t$.

We will show that the energies $E^{\alpha}$ are coercive in $W^{\frac{\alpha+1}{2}, 2}$ (cf. Theorem 3.2) in Sect. 3.1. Together with the above inequalities this implies that the $W^{\frac{\alpha-1}{2}}, 2$ norm of the unit tangents of the curve is uniformly bounded.

To get higher order estimates, we calculate the evolution equations of the terms

$$
\mathcal{E}^{k}=\int_{\mathbb{R} / \mathbb{Z}}\left|\partial_{s}^{k} \kappa\right|^{2} d s
$$

(cf. 3.3) in Sect. 3.2 and show that the resulting terms can be estimated using GagliardoNirenberg-Sobolev inequalities for fractional Sobolev spaces and Besov spaces.

In the Sect. 3.4 we put all these pieces together to show that the solution to the flow exists for all time and subconverges after suitable translations and re-parameterizations if necessary to a critical point.

\subsection{Coercivity of the energy}

Theorem 1.1 in [5] states that for curves parameterized by arc length, the energy $E^{\alpha}$ is finite if and only if the curve is injective and belongs to $W^{\frac{\alpha+1}{2}, 2}\left(\mathbb{R} / \mathbb{Z}, \mathbb{R}^{n}\right)$.

One of the most important ingredients in the proof of the long time existence result is the following quantitative version of the regularizing effects of Theorem 1.1 in [5]:

Theorem 3.2 (Coercivity of $E^{\alpha}$ ) Let $c \in C^{1}\left(\mathbb{R} / l \mathbb{Z}, \mathbb{R}^{n}\right), l>0$, be a curve parametrized by arc length and $\alpha \in[2,3)$. Then there exists a constant $C=C(\alpha)<$ $\infty$ depending only on $\alpha$ such that

$$
\left|c^{\prime}\right|_{W \frac{\alpha-1}{2}, 2} \leq C E^{\alpha}(c)
$$

Proof We have

$$
\begin{aligned}
E^{\alpha}(c) & =\int_{\mathbb{R} / l \mathbb{Z}} \int_{-\frac{l}{2}}^{\frac{l}{2}}\left(\frac{1}{|c(u+w)-c(u)|^{\alpha}}-\frac{1}{|w|^{\alpha}}\right) d w d u \\
& =\int_{\mathbb{R} / l \mathbb{Z}} \int_{-\frac{l}{2}}^{\frac{l}{2}} \frac{|w|^{\alpha}}{|c(u+w)-c(u)|^{\alpha}}\left(\frac{1-\frac{|c(u+w)-c(u)|^{\alpha}}{|w|^{\alpha}}}{|w|^{\alpha}}\right) d w d u
\end{aligned}
$$




$$
\begin{aligned}
& \underset{\mathbb{R}(u+w)-c(u)|\leq| w \mid}{\geq} \int_{\mathbb{R} / l \mathbb{Z}} \int_{-\frac{l}{2}}^{\frac{l}{2}}\left(\frac{1-\frac{|c(u+w)-c(u)|^{\alpha}}{|w|^{\alpha}}}{|w|^{\alpha}}\right) d w d u \\
& 1-a^{\alpha} \geq 1-a^{2} \text { for } a \in[0,1] \int_{\mathbb{R} / l \mathbb{Z}} \int_{-\frac{l}{2}}^{\frac{l}{2}}\left(\frac{1-\frac{|c(u+w)-c(u)|^{2}}{|w|^{2}}}{|w|^{\alpha}}\right) d w d u \\
& =\int_{\mathbb{R} / l \mathbb{Z}} \int_{-\frac{l}{2}}^{\frac{l}{2}}\left(\frac{1-\int_{0}^{1} \int_{0}^{1}\left\langle c^{\prime}\left(u+\tau_{1} w\right), c^{\prime}\left(u+\tau_{2} w\right)\right\rangle d \tau_{1} d \tau_{2}}{|w|^{\alpha}}\right) d w d u \\
& \stackrel{\left|c^{\prime}\right|=1}{=} \frac{1}{2} \int_{\mathbb{R} / l \mathbb{Z}} \int_{-\frac{l}{2}}^{\frac{l}{2}}\left(\frac{\int_{0}^{1} \int_{0}^{1}\left|c^{\prime}\left(u+\tau_{1} w\right)-c^{\prime}\left(u+\tau_{2} w\right)\right|^{2} d \tau_{1} d \tau_{2}}{|w|^{\alpha}}\right) d w d u .
\end{aligned}
$$

Using Fubini and successively substituting $u$ by $u+\tau_{1} w$ and then $w$ by $\left(\tau_{2}-\tau_{1}\right) w$, we get

$$
\begin{aligned}
E^{\alpha}(c) & \geq \int_{\mathbb{R} / l \mathbb{Z}} \int_{-\frac{l}{2}}^{\frac{l}{2}} \int_{0}^{1} \int_{0}^{1}\left(\frac{\left|c^{\prime}\left(u+\tau_{1} w\right)-c^{\prime}\left(u+\tau_{2} w\right)\right|^{2}}{|w|^{\alpha}}\right) d \tau_{1} d \tau_{2} d w d u \\
& \geq \int_{0}^{1} \int_{0}^{1}\left(\left|\tau_{2}-\tau_{1}\right|^{\alpha-1} \int_{\mathbb{R}^{\prime} / l \mathbb{Z}} \int_{-\frac{\left|\tau_{2}-\tau_{1}\right| l}{2}}^{\frac{\left|\tau_{2}-\tau_{1}\right| l}{2}}\left(\frac{\left|c^{\prime}(u)-c^{\prime}(u+w)\right|^{2}}{|w|^{\alpha}}\right) d w d u\right) d \tau_{1} d \tau_{2} \\
& \geq\left(\frac{1}{2}\right)^{\alpha-1} \int_{0}^{1 / 4} \int_{3 / 4}^{1} \int_{\mathbb{R} / l \mathbb{Z}}^{1} \int_{-\frac{l}{4}}^{\frac{l}{4}}\left(\frac{\left|c^{\prime}(u)-c^{\prime}(u+w)\right|^{2}}{|w|^{\alpha}}\right) d w d u d \tau_{1} d \tau_{2} \\
& \geq\left(\frac{1}{2}\right)^{\alpha+3} \int_{\mathbb{R} / l \mathbb{Z}}^{\frac{l}{4}} \int_{-\frac{l}{4}}^{\frac{l}{4}}\left(\frac{\left|c^{\prime}(u)-c^{\prime}(u+w)\right|^{2}}{|w|^{\alpha}}\right) d w d u
\end{aligned}
$$


and finally

$$
\begin{aligned}
\left|c^{\prime}\right|_{W^{\frac{\alpha-1}{2}}}^{2}= & \int_{\mathbb{R} / l \mathbb{Z}} \int_{-\frac{l}{2}}^{\frac{l}{2}}\left(\frac{\left|c^{\prime}(u)-c^{\prime}(u+w)\right|^{2}}{|w|^{\alpha}}\right) d w d u \\
\leq & C \int_{\mathbb{R} / l \mathbb{Z}} \int_{-\frac{l}{2}}^{\frac{l}{2}}\left(\frac{\left|c^{\prime}(u)-c^{\prime}(u+w / 2)\right|^{2}}{|w|^{\alpha}}\right) d w d u \\
& +C \int_{\mathbb{R} / l \mathbb{Z}} \int_{-\frac{l}{2}}^{\frac{l}{2}}\left(\frac{\left|c^{\prime}(u+w / 2)-c^{\prime}(u+w)\right|^{2}}{|w|^{\alpha}}\right) d w d u \\
\leq & C \int_{\mathbb{R} / l \mathbb{Z}} \int_{-\frac{l}{4}}^{\frac{l}{4}}\left(\frac{\left|c^{\prime}(u)-c^{\prime}(u+w)\right|^{2}}{|w|^{\alpha}}\right) d w d u \\
\leq & C E^{\alpha}(c) .
\end{aligned}
$$

\subsection{Evolution equations of higher order energies}

As for most of our estimates the precise algebraic form of the terms does not matter, we will use the following notation to describe the essential structure of the terms.

For two Euclidean vectors $v, w$, the term $v * w$ stands for a bilinear operator in $v$ and $w$ into another Euclidean vector space. For a regular curve $c$, let $\partial_{s}=\frac{\partial_{x}}{\left|c^{\prime}\right|}$ denote the derivative with respect to arc length denoted by $s$ and let $\tau=\partial_{s} c=\frac{c^{\prime}}{\left|c^{\prime}\right|}$ be the unit tagent vector along $c$. For $\mu, v \in \mathbb{N}$, a regular curve $c \in C^{\infty}\left(\mathbb{R} / \mathbb{Z}, \mathbb{R}^{n}\right)$ and a function $f: \mathbb{R} / \mathbb{Z} \rightarrow \mathbb{R}^{k}$ we let $P_{v}^{\mu}(f)$ be a linear combination of terms of the form $\partial_{s}^{j_{1}} f * \cdots * \partial_{s}^{j_{v}} f, j_{1}+\cdots+j_{v}=\mu$. Furthermore, given a second function $g: \mathbb{R} / \mathbb{Z} \rightarrow \mathbb{R}^{k}$ the expression $P_{\nu}^{\mu}(g, f)$ denotes a linear combination of terms of the form $\partial_{s}^{j_{1}} g * \partial_{s}^{j_{2}} f * \partial_{s}^{j_{3}} f * \cdots * \partial_{s}^{j_{v}} f, j_{1}+\cdots+j_{v}=\mu$.

Let $c_{t}$ be a smooth family of smooth closed curves moving only in normal direction, i.e., $V:=\partial_{t} c_{t}$ is normal along $c_{t}$. For the convenience of the reader let us derive some basic evolution equations which in similar forms can be found in (cf. [12]. Using that $V$ is pointing in a normal direction we get

$$
\begin{aligned}
\partial_{t}\left(\left|c^{\prime}\right|\right) & =\left\langle\frac{c^{\prime}}{\left|c^{\prime}\right|}, \partial_{x} \partial_{t} c\right\rangle=\left\langle\tau, \partial_{x} V\right\rangle=\partial_{x}\langle\tau, V\rangle-\left\langle\partial_{x} \tau, V\right\rangle \\
& =-\langle\kappa, V\rangle\left|c^{\prime}\right| .
\end{aligned}
$$


Hence,

$$
\begin{aligned}
\partial_{t} \partial_{s} & =\partial_{t}\left(\frac{\partial_{x}}{\left|c^{\prime}\right|}\right)=\frac{\partial_{x} \partial_{t}}{\left|c^{\prime}\right|}-\frac{\left(\partial_{t}\left|c^{\prime}\right|\right)}{\left|c^{\prime}\right|^{2}} \partial_{x} \\
& =\partial_{s} \partial_{t}+\langle\kappa, V\rangle \partial_{s}
\end{aligned}
$$

and thus

$$
\partial_{t} \tau=\partial_{t} \partial_{s} c=\partial s \partial_{t} c+\langle\kappa, V\rangle \partial_{s} c=\partial_{s} V+\langle\kappa, V\rangle \tau
$$

Furthermore,

$$
\begin{aligned}
\partial_{t} \kappa & =\partial_{t} \partial_{s} \tau=\partial_{s} \partial_{t} \tau+\langle\kappa, V\rangle \partial_{s} \tau \\
& =\partial_{s}^{2} V+\partial_{s}(\langle\kappa, V\rangle \tau)+\langle\kappa, V\rangle \kappa .
\end{aligned}
$$

Using these equations, we inductively deduce the following evolution equations of arbitrary derivatives of the curvature.

Lemma 3.3 Let $I \subset \mathbb{R}$ be open and $c: \mathbb{R} / l \mathbb{Z} \times I \rightarrow \mathbb{R}$ be a smooth family of curves such that $V:=\partial_{t} c$ is normal along $c$, i.e. $\left\langle V(s, t), c^{\prime}(s, t)\right\rangle=0$. Then

$$
\partial_{t}\left(\partial_{s}^{k} \kappa\right)=\partial_{s}^{k+2} V+\partial_{s}\left(P_{2}^{k}(V, \kappa) \tau\right)+P_{3}^{k}(V, \kappa)
$$

for all $k \in \mathbb{N}_{0}$.

Proof By (3.4) the statement is true for $k=0$. If the statement was true for some $k$ then

$$
\begin{aligned}
\partial_{t}\left(\partial_{s}^{k+1} \kappa\right) & =\partial_{s}\left(\partial_{t}\left(\partial_{s}^{k} \kappa\right)\right)+\langle\kappa, V\rangle \partial_{s}^{k+1} \kappa \\
& =\partial_{s}\left(\partial_{s}^{k+2} V+\partial_{s}\left(P_{2}^{k}(V, \kappa) \tau\right)+P_{3}^{k}(V, \kappa)\right)+\langle\kappa, V\rangle \partial_{s}^{k+1} \kappa \\
& =\partial_{s}\left(\partial_{s}^{k+2} V+P_{2}^{k+1}(V, \kappa) \tau+P_{3}^{k}(V, \kappa)+P_{3}^{k}(V, \kappa)\right)+\langle\kappa, V\rangle \partial_{s}^{k+1} \kappa \\
& =\partial_{s}^{(k+1)+2} V+\partial_{s}\left(P_{2}^{k+1}(V, \kappa) \tau\right)+P_{3}^{k+1}(V, \kappa) .
\end{aligned}
$$

Hence, induction gives the assertion.

An immediate corollary of Lemma 3.3 and (3.2) is the following.

Corollary 3.4 Let c be a family of curves moving with normal speed $V$. Then

$$
\begin{aligned}
\partial_{t} \int_{\mathbb{R} / \mathbb{Z}}\left|\partial_{s}^{k} \kappa\right|^{2} d s= & 2 \int_{\mathbb{R} / \mathbb{Z}}\left\langle\partial_{s}^{k+2} V, \partial_{s}^{k} \kappa\right\rangle d s+2 \int_{\mathbb{R} / \mathbb{Z}}\left\langle P_{2}^{k}(V, \kappa) \tau, \partial_{s}^{k+1} \kappa\right\rangle d s \\
& +2 \int_{\mathbb{R} / \mathbb{Z}}\left\langle P_{3}^{k}(V, \kappa), \partial_{s}^{k} \kappa\right\rangle d s-\int_{\mathbb{R} / \mathbb{Z}}\left|\partial_{s}^{k} \kappa\right|^{2}\langle\kappa, V\rangle d s .
\end{aligned}
$$


Proof We have

$$
\begin{aligned}
\partial_{t} \int_{\mathbb{R} / \mathbb{Z}}\left|\partial_{s}^{k} \kappa\right|^{2} d s & =\partial_{t} \int_{\mathbb{R} / \mathbb{Z}}\left|\partial_{s}^{k} \kappa\right|^{2}\left|c^{\prime}\right| d x \\
& =\int_{\mathbb{R} / \mathbb{Z}}\left(2\left\langle\partial_{s}^{k} \kappa, \partial_{t} \partial_{s}^{k} \kappa\right\rangle\left|c^{\prime}\right|+\left|\partial_{s}^{k} \kappa\right|^{2} \partial_{t}\left(\left|c^{\prime}\right|\right) d x\right.
\end{aligned}
$$

Together with Lemma 3.3 and (3.2) we get

$$
\begin{aligned}
\partial_{t} \int_{\mathbb{R} / \mathbb{Z}}\left|\partial_{s}^{k} \kappa\right|^{2} d s= & 2 \int_{\mathbb{R} / \mathbb{Z}}\left\langle\partial_{s}^{k+2} V, \partial_{s}^{k} \kappa\right\rangle d s+2 \int\left\langle\partial_{s}\left(P_{2}^{k}(V, \kappa) \tau\right), \partial_{s}^{k} \kappa\right\rangle d s \\
& +2 \int\left\langle P_{3}^{k}(V, \kappa), \partial_{s}^{k} \kappa\right\rangle d s-\int\left|\partial_{s}^{k} \kappa\right|^{2}\langle\kappa, V\rangle d s
\end{aligned}
$$

Partial integration for the second term on the left hand side yields the claim.

\subsection{Interpolation estimates}

In this section we will prove several estimates that will be needed later in the proof of the long time existence result. In a natural way the Besov spaces $B_{q}^{s, p}$ appear during our calculations.

Lemma 3.5 (Gagliardo-Nirenberg-Sobolev type estimates for a typical term) For $\alpha \in[2,3)$ let $j_{1}, \ldots, j_{v+2} \in \mathbb{N}, j_{1}, \ldots, j_{v} \geq 2$, and $s>\frac{3}{2}$ be such that there are $p_{1}, \ldots, p_{v+2} \in[1, \infty]$ with

$$
\sum_{i=1}^{v+2} \frac{1}{p_{i}}=1,
$$

$\frac{\alpha}{2} \leq j_{i}-\frac{1}{p_{i}} \leq s+\frac{\alpha}{2}$ for $i=1, \ldots, v$ and $j_{i}-\frac{1}{p_{i}} \leq s+\frac{1}{2}$ for $i=v+1, v+2$. Let $\theta:=\left(\sum_{i=1}^{v+2} j_{i}-v \frac{\alpha}{2}-2\right) / s$.

Then for all $\Lambda<\infty$ there is a $C=C(\Lambda)$ such that

$$
\begin{aligned}
& \int_{\mathbb{R} / l \mathbb{Z}} \int_{-\frac{l}{2}}^{\frac{l}{2}}\left(\prod_{i=1}^{v}\left|\partial^{j_{i}} f\left(u+\sigma_{i} w\right)\right|\right) \\
& \quad \times\left(\prod_{i=v+1}^{v+2} \frac{\int_{0}^{1} \int_{0}^{1}\left|\partial^{j_{i}} f\left(u+\tau_{1} w\right)-\partial^{j_{i}} f\left(u+\tau_{2} w\right)\right| d \tau_{1} d \tau_{2}}{|w|^{\alpha}}\right) d w d u \\
& \leq C\|f\|_{W^{\frac{\alpha+1}{2}+s, 2}}^{\theta}\|f\|_{W^{\frac{\alpha+1}{2}, 2}}^{\nu+2-\theta}
\end{aligned}
$$

holds for all $f \in C^{\infty}\left(\mathbb{R} / l \mathbb{Z}, \mathbb{R}^{n}\right)$ if $\Lambda^{-1} \leq l \leq \Lambda, \sigma_{i} \in \mathbb{R}$. 
Proof Using Hölder's inequality for the integration with respect to $u$, we get

$$
\begin{aligned}
& \int_{\mathbb{R} / l \mathbb{Z}} \int_{-\frac{l}{2}}^{\frac{l}{2}}\left(\prod_{i=1}^{v}\left|\partial_{s}^{j_{i}} f\left(u+\sigma_{i} w\right)\right|\right) \\
& \quad \times\left(\prod_{i=v+1}^{v+2} \frac{\int_{0}^{1} \int_{0}^{1}\left|\partial^{j_{i}} f\left(u+\tau_{1} w\right)-\partial^{j_{i}} f\left(u+\tau_{2} w\right)\right| d \tau_{1} d \tau_{2}}{|w|^{\alpha}}\right) d w d u \\
& \leq\left(\prod_{i=1}^{v}\left\|\partial^{j_{i}} f\right\|_{L^{p_{i}}}\right) \int_{0}^{1} \int_{0}^{1} \int_{-\frac{l}{2}}^{\frac{l}{2}} \frac{\prod_{i=v+1}^{v+2}\left\|\partial^{j_{i}} f\left(\cdot+\left(\tau_{1}-\tau_{2}\right) w\right)-\partial^{j_{i}} f\right\|_{L^{p_{i}}}}{|w|^{\alpha}} d w d \tau_{1} d \tau_{2} \\
& \leq\left(\prod_{i=1}^{v}\left\|\partial^{j_{i}} f\right\|_{L^{p_{i}}}\right) \int_{0}^{1} \int_{0}^{1} \prod_{i=v+1}^{v+2}\left(\int_{-\frac{l}{2}}^{\frac{l}{2}} \frac{\left\|\partial^{j_{i}} f\left(\cdot+\left(\tau_{1}-\tau_{2}\right) w\right)-\partial^{j_{i}} f\right\|_{L^{p_{i}}}^{2}}{|w|^{\alpha}} d w\right)^{\frac{1}{2}} d \tau_{1} d \tau_{2} .
\end{aligned}
$$

Substituting $w$ by $\left(\tau_{1}-\tau_{2}\right) w$ we can estimate this further by

$$
\begin{aligned}
& \leq C\left(\prod_{i=1}^{v}\left\|\partial^{j_{i}} f\right\|_{L^{p_{i}}}\right) \int_{0}^{1} \int_{0}^{1}\left|\tau_{1}-\tau_{2}\right|^{\alpha-1} \\
& \times \prod_{i=v+1}^{v+2}\left(\int_{-\frac{l}{2}}^{\frac{l}{2}} \frac{\left\|\partial^{j_{i}} f(\cdot+w)-\partial^{j_{i}} f\right\|_{L^{p_{i}}}^{2}}{|w|^{\alpha}} d w\right)^{\frac{1}{2}} d \tau_{1} d \tau_{2}
\end{aligned}
$$

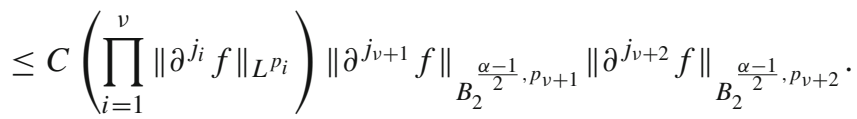

Scaling the Gagliardo-Nirenberg-Sobolev estimates (Theorem 8.1), the last term can be estimated from above by

$$
C \prod_{i=1}^{\nu+2}\|f\|_{W^{s+\frac{\alpha+1}{2}, 2}}^{\theta_{i}}\|f\|_{W^{\frac{\alpha+1}{2}, 2}}^{1-\theta_{i}}
$$

where $\theta_{i}:=\frac{\left(j_{i}-\frac{1}{p_{i}}\right)-\frac{\alpha}{2}}{s}$ for $i=1, \ldots, v$ and $\theta_{i}:=\frac{\left(j_{i}-\frac{1}{p_{i}}\right)-\frac{1}{2}}{s}$ for $i=v+1, v+2$. Thus the assertion of the theorem follows.

We will now use the lemma above to estimate a typical term that repeatedly will appear in the later calculations.

Lemma 3.6 (Estimates for terms containing the energy integrand) For all $\Lambda<\infty$ there is a $C(\Lambda)<\infty$ such that the following holds: 
Let $\Lambda^{-1} \leq l \leq \Lambda$ and $c \in C^{\infty}\left(\mathbb{R} / l \mathbb{Z}, \mathbb{R}^{n}\right)$ be a curve parameterized by arc length satisfying the bi-Lipschitz estimate

$$
|w| \leq \Lambda|c(u+w)-c(u)|, \quad \forall u \in \mathbb{R} / l \mathbb{Z}, w \in[-l / 2, l / 2]
$$

Then for $\beta \geq 0$ the functions

$$
g_{\beta}: \mathbb{R} / l \mathbb{Z} \rightarrow \mathbb{R}, \quad g_{\beta}(s):=\int_{-\frac{l}{2}}^{\frac{l}{2}}\left(\frac{|w|^{\beta}}{|c(s+w)-c(s)|^{\alpha+\beta}}-\frac{|w|^{\beta}}{|w|^{\alpha+\beta}}\right) d w
$$

are in $C^{\infty}(\mathbb{R} / l \mathbb{Z}, \mathbb{R})$ for $\beta>0$. If furthermore $\mu \leq s+\frac{\alpha}{2}$ and $k+1 \leq s$, we have

$$
\begin{array}{r}
\int_{\mathbb{R} / l \mathbb{Z}}\left|\partial_{s}^{k}\left\{\int_{-\frac{l}{2}}^{\frac{l}{2}}\left(\frac{|w|^{\beta}}{|c(s+w)-c(s)|^{\alpha+\beta}}-\frac{|w|^{\beta}}{|w|^{\alpha+\beta}}\right) d w\right\} P_{v}^{\mu}\left(c^{\prime}\right)(s)\right| d s \\
\leq C \sum_{m=1}^{k}\|c\|_{W^{\frac{\alpha+1}{2}+s, 2}\left(\mathbb{R} / l \mathbb{Z}, \mathbb{R}^{n}\right)}^{\theta_{m}}\|c\|_{W^{\frac{\alpha+1}{2}, 2}\left(\mathbb{R} / l \mathbb{Z}, \mathbb{R}^{n}\right)}^{m+v+2-\theta_{m}}
\end{array}
$$

where $\theta_{m}:=\left(k+(m+2)+\mu+v-(m+v) \frac{\alpha}{2}-2\right) / s<(k+\mu) / s$. If $(k+\mu) / s \leq 2$, this implies that for every $\varepsilon>0$ there is a constant $C\left(\varepsilon,\|c\|_{W^{\frac{\alpha+1}{2}, 2}\left(\mathbb{R} / l \mathbb{Z}, \mathbb{R}^{n}\right)}\right)<\infty$ such that

$$
\begin{aligned}
& \int_{\mathbb{R} / l \mathbb{Z}}\left|\partial_{s}^{k}\left\{\int_{-\frac{l}{2}}^{\frac{l}{2}}\left(\frac{|w|^{\beta}}{|c(s+w)-c(s)|^{\alpha+\beta}}-\frac{|w|^{\beta}}{|w|^{\alpha+\beta}}\right) d w\right\} P_{v}^{\mu}\left(c^{\prime}\right)(s)\right| d s \\
& \leq \varepsilon\left\|(-\Delta)^{\frac{\alpha+1}{4}+\frac{s}{2}} c\right\|_{L^{2}\left(\mathbb{R} / l \mathbb{Z}, \mathbb{R}^{n}\right)}^{2}+C\left(\varepsilon,\|c\|_{W^{\frac{\alpha+1}{2}, 2}\left(\mathbb{R} / l \mathbb{Z}, \mathbb{R}^{n}\right)}\right)
\end{aligned}
$$

Proof Note that $\partial_{s}=\partial_{x}$ since $c$ is parametrized by arc-length. For $\frac{l}{2}>\varepsilon>0$ and $I_{l, \varepsilon}=\left[-\frac{l}{2}, \frac{l}{2}\right] \backslash[-\varepsilon, \varepsilon]$ we set

$$
g_{\beta}^{(\varepsilon)}(s):=\int_{w \in I_{l, \varepsilon}} h_{\beta}(s, w) d w
$$

and

$$
h_{\beta}(s, w):=\frac{|w|^{\beta}}{|c(s+w)-c(s)|^{\alpha+\beta}}-\frac{|w|^{\beta}}{|w|^{\alpha+\beta}}
$$


for all $s \in \mathbb{R} / l \mathbb{Z}$ and $w \in[-l / 2, l / 2], w \neq 0$. Then due to the bi-Lipschitz estimate for $c$ we have $g_{\beta}^{(\varepsilon)} \in C^{\infty}\left(\mathbb{R} / l \mathbb{Z}, \mathbb{R}^{n}\right)$ and

$$
\partial_{s}^{k} g_{\beta}^{(\varepsilon)}(s)=\int_{w \in I_{l, \varepsilon}} \partial_{s}^{k} h_{\beta}(s, w) d w
$$

With $G_{\beta}(v):=\frac{1}{|v|^{\alpha+\beta}} \frac{1-|v|^{\alpha+\beta}}{1-|v|^{2}}$ we get

$$
\begin{gathered}
\frac{|w|^{\beta}}{|c(s+w)-c(s)|^{\alpha+\beta}}-\frac{|w|^{\beta}}{|w|^{\alpha+\beta}}=G_{\beta}\left(\frac{c(s+w)-c(s)}{w}\right) \cdot \frac{1-\frac{|c(s+w)-c(s)|^{2}}{w^{2}}}{|w|^{\alpha}} \\
=\frac{1}{2} G_{\beta}\left(\frac{c(s+w)-c(s)}{w}\right) \cdot \int_{0}^{1} \int_{0}^{1} \frac{\left|c^{\prime}\left(s+\tau_{1} w\right)-c^{\prime}\left(s+\tau_{2} w\right)\right|^{2}}{|w|^{\alpha}} d \tau_{1} d \tau_{2} .
\end{gathered}
$$

Note that $G_{\beta}$ is a smooth function on $\mathbb{R}^{n} \backslash\{0\}$. Since for $s \in \mathbb{R} / l \mathbb{Z}, w \in[-l / 2, l / 2]-$ $\{0\}$ we have $1 \geq\left|\frac{c(s+w)-c(s)}{w}\right| \geq \Lambda^{-1}$, for every $k \in \mathbb{N}_{0}$ there exists a constant $C<\infty$ such that

$$
\left|\left(D^{k} G_{\beta}\right)\left(\frac{c(s+w)-c(s)}{w}\right)\right| \leq C \quad \forall s \in \mathbb{R} / l \mathbb{Z}, w \in[-l / 2, l / 2]-\{0\} .
$$

Using the product rule together with Fáa di Bruno's formula for higher derivatives of composite functions, we conclude that

$$
\partial_{s}^{k} h_{\beta}(s, w)=\partial_{s}^{k}\left(\frac{|w|^{\beta}}{|c(s+w)-c(s)|^{\alpha+\beta}}-\frac{|w|^{\beta}}{|w|^{\alpha+\beta}}\right)
$$

is a linear combination of terms $T_{k_{1}, \ldots, k_{m+2}}$ of the form

$$
\begin{aligned}
& D^{m} G_{\beta}\left(\frac{c(s+w)-c(s)}{w}\right)\left(\frac{\partial_{s}^{k_{1}} c(s+w)-\partial_{s}^{k_{1}} c(s)}{w}, \ldots, \frac{\partial_{s}^{k_{m}} c(s+w)-\partial_{s}^{k_{m}} c(s)}{w}\right) \\
& \times \int_{0}^{1} \int_{0}^{1} \frac{\left\langle\partial_{s}^{k_{m+1}+1} c\left(s+\tau_{1} w\right)-\partial_{s}^{k_{m+1}+1} c\left(s+\tau_{2} w\right), \partial_{s}^{k_{m+2}+1} c\left(s+\tau_{1} w\right)-\partial_{s}^{k_{m+2}+1} c\left(s+\tau_{2} w\right)\right\rangle}{|w|^{\alpha}} d \tau_{1} d \tau_{2}
\end{aligned}
$$

where $m, k_{1}, \ldots k_{m+2} \in \mathbb{N}_{0}, m \leq k, k_{1}, \ldots k_{m} \geq 1$, and

$$
\sum_{i=1}^{m+2} k_{i}=k
$$


Using (3.7) and the fundamental theorem of calculus, the absolute value of such terms is bounded by

$$
\begin{aligned}
& C \prod_{i=1}^{m}\left|\int_{0}^{1} \partial_{s}^{k_{i}+1} c\left(s+\sigma_{i} w\right) d \sigma_{i}\right| \\
& \quad \times\left|\int_{0}^{1} \int_{0}^{1} \frac{\left\langle\partial_{s}^{k_{m+1}+1} c\left(s+\tau_{1} w\right)-\partial_{s}^{k_{m+2}+1} c\left(s+\tau_{2} w\right), \partial_{s}^{k_{m+2}+1} c\left(s+\tau_{1} w\right)-\partial_{s}^{k_{m+2}+1} c\left(s+\tau_{2} w\right)\right\rangle}{|w|^{\alpha}} d \tau_{1} d \tau_{2}\right| \\
& \leq \frac{C\|c\|_{C^{k+2}}^{m+2}}{|w|^{\alpha-2}} .
\end{aligned}
$$

Hence,

$$
\left|\partial_{s}^{k} h_{\beta}(s, w)\right| \leq \frac{C\|c\|_{C^{k+2}}^{k+2}}{|w|^{\alpha-2}} .
$$

From this we deduce that for $\varepsilon_{1}>\varepsilon_{2}>0$ we have

$$
\begin{aligned}
\left|\partial_{s}^{k} g_{\beta}^{\left(\varepsilon_{1}\right)}(s)-\partial_{s}^{k} g_{\beta}^{\left(\varepsilon_{2}\right)}(s)\right| & \leq \int_{w \in\left[-\varepsilon_{1}, \varepsilon_{1}\right] \backslash\left[-\varepsilon_{2}, \varepsilon_{2}\right]}\left|\partial_{s}^{k} h_{\beta}(s, w)\right| d w \\
& \leq C\|c\|_{C^{k+2}}^{k+2} \int_{\varepsilon_{2}}^{\varepsilon_{1}} \frac{1}{|w|^{\alpha-2}} d w \leq C\|c\|_{C^{k+2}}^{m+2} \varepsilon_{1}^{3-\alpha}
\end{aligned}
$$

As $\alpha<3, g_{\beta}^{(\varepsilon)}$ converges smoothly to a smooth representative of $g_{\beta}$ and

$$
\partial_{s}^{k} g_{\beta}(s)=\int_{-\frac{l}{2}}^{\frac{l}{2}} \partial_{s}^{k} h_{\beta}(s, w) d w=\int_{-\frac{l}{2}}^{\frac{l}{2}} \partial_{s}^{k}\left(\frac{|w|^{\beta}}{|c(s+w)-c(s)|^{\alpha+\beta}}-\frac{|w|^{\beta}}{|w|^{\alpha+\beta}}\right) d w .
$$

Using that $\partial_{s}^{k} h_{\beta}(s, w)$ is a linear combination of terms like (3.8) together with Lemma 3.5 , we obtain

$$
\int_{\mathbb{R} / l \mathbb{Z}}\left|\left(\partial_{s}^{k} g_{\beta}(s)\right) P_{\nu}^{\mu}\left(c^{\prime}\right)(s)\right| d s \leq C \sum_{m=1}^{k}\|c\|_{W^{\frac{\alpha+1}{2}+s, 2}\left(\mathbb{R} / l \mathbb{Z}, \mathbb{R}^{n}\right)}^{\theta_{m}}\|c\|_{W^{\frac{\alpha+1}{2}, 2}\left(\mathbb{R} / l \mathbb{Z}, \mathbb{R}^{n}\right)}^{m+v+2-\theta_{m}}
$$

where $\theta_{m}:=\left(k+(m+2)+\mu+v-(m+v) \frac{\alpha}{2}-2\right) / s<(k+m+\mu+v-m-v) / s \leq$ $(k+\mu) / s$. This proves inequality (3.5) from which one obtains (3.6) using CauchySchwartz.

\subsection{Proof of long time existence}

First we will derive the following estimate from the evolution equation of the higher order energies in Corollary 3.4 using Lemma 3.6. For a periodic function $f \in C^{\infty}\left(\mathbb{R} / l \mathbb{Z}, \mathbb{R}^{n}\right)$ we use the shorthand 


$$
D^{s} f=(-\Delta)^{\frac{s}{2}} f
$$

for the fractional Laplacian.

Lemma 3.7 For every $k \in \mathbb{N}$ and $\varepsilon>0$ there are constants $C_{\varepsilon}<\infty$ such that

$$
\partial_{t} \int_{\mathbb{R} / \mathbb{Z}}\left|\partial_{s}^{k} \kappa_{c_{t}}(s)\right|^{2} d s+c_{\alpha} \int_{\mathbb{R} / \mathbb{Z}}\left|\left(D_{s}\right)^{k+\frac{\alpha+1}{2}} \kappa\right| d s \leq \varepsilon \int_{\mathbb{R} / \mathbb{Z}}\left|\left(D_{s}\right)^{k+\frac{\alpha+1}{2}} \kappa\right|^{2} d s+C_{\varepsilon} .
$$

Proof Corollary 3.4 tells us that

$$
\begin{aligned}
& \partial_{t} \int_{\mathbb{R} / \mathbb{Z}}\left|\partial_{s}^{k} \kappa\right|^{2} d s=2 \int_{\mathbb{R} / \mathbb{Z}}\left\langle\partial_{s}^{k+2} V, \partial_{s}^{k} \kappa\right\rangle d s+2 \int_{\mathbb{R} / \mathbb{Z}}\left\langle P_{2}^{k}(V, \kappa) \tau, \partial_{s}^{k+1} \kappa\right\rangle d s \\
& \quad+2 \int_{\mathbb{R} / \mathbb{Z}}\left\langle P_{3}^{k}(V, \kappa), \partial_{s}^{k} \kappa\right\rangle d s-\int_{\mathbb{R} / \mathbb{Z}}\left|\partial_{s}^{k} \kappa\right|^{2}\langle\kappa, V\rangle d s
\end{aligned}
$$

where $V=-\mathfrak{V}^{\alpha} c+\lambda \kappa=-P_{c^{\prime}}^{\perp} \tilde{\mathfrak{V}}^{\alpha} c+\lambda \kappa$.

Let us now fix the time $t$ and let us re-parameterize $c_{t}$ for this fixed time by arc length to estimate the right-hand side of this equation and let $l$ denote the length of the curve at time $t$.

We decompose ${ }^{2}$

$$
\tilde{\mathfrak{V}}^{\alpha} c=\alpha Q^{\alpha} c-2 R_{1}^{\alpha} c+2 \alpha R_{2}^{\alpha} c
$$

where

$$
\begin{aligned}
\left(Q^{\alpha} c\right)(s) & \left.:=p \cdot v \cdot \int_{-\frac{l}{2}}^{\frac{l}{2}}\left\{2 \frac{c(s+w)-c(s)-w c^{\prime}(s)}{|w|^{2}}-c^{\prime \prime}(s)\right)\right\} \frac{d w}{|w|^{\alpha}}, \\
\left(R_{1}^{\alpha} c\right)(s):= & \int_{-\frac{l}{2}}^{\frac{l}{2}} c^{\prime \prime}(s)\left(\frac{1}{|c(s+w)-c(s)|^{\alpha}}-\frac{1}{|w|^{\alpha}}\right) d w, \\
\left(R_{2}^{\alpha} c\right)(s):= & \int_{-\frac{l}{2}}^{\frac{l}{2}}\left(c(s+w)-c(s)-w c^{\prime}(s)\right)\left(\frac{1}{|c(s+w)-c(s)|^{\alpha+2}}-\frac{1}{|w|^{\alpha+2}}\right) d w .
\end{aligned}
$$

\footnotetext{
2 Since the curve now is parameterized by arc-length, the decompostion we use here is much simpler than the one given in (2.2) - though still we get it in the same spirit.
} 
Using the fundamental theorem of calculus, we rewrite $\left(R_{2}^{\alpha} c\right)(s)$ as

$$
\left(R_{2}^{\alpha} c\right)(s):=\int_{0}^{1}\left(1-\tau_{1}\right) \int_{-\frac{l}{2}}^{\frac{l}{2}} c^{\prime \prime}\left(s+\tau_{1} w\right)\left(\frac{w^{2}}{|c(s+w)-c(s)|^{\alpha+2}}-\frac{w^{2}}{|w|^{\alpha+2}}\right) d w d \tau_{1} .
$$

and set

$$
R^{\alpha} c:=-2 R_{1}^{\alpha} c+2 \alpha R_{2}^{\alpha} c .
$$

Hence,

$$
V=-P_{c^{\prime}}^{\perp}\left(\alpha Q^{\alpha} c+R^{\alpha} c\right)+\lambda \kappa
$$

Now we first estimate all the terms appearing in (3.9) after plugging into it the decomposition (3.11) except for the term

$$
\int_{\mathbb{R} / l \mathbb{Z}}\left\langle\partial_{s}^{k+2} P_{c^{\prime}}^{\perp} Q^{\alpha} c, \partial_{s}^{k} \kappa\right\rangle d s
$$

using Hölder's inequality together with the standard Gagliardo-Nirenberg-Sobolev inequality or the version in Lemma 3.5. We get that for all $\varepsilon>0$ there is a constant $C_{\varepsilon}<\infty$ such that all these terms can be estimated from above by

$$
\varepsilon\left\|D^{k+2+\frac{\alpha+1}{2}} c\right\|_{L^{2}}^{2}+C_{\varepsilon} .
$$

We will only give the details for some exemplary terms as all the other terms can be estimated following the same line of arguments. We start by estimating the terms containing the remainder $R^{\alpha}$. For the first term in Eq. (3.9) we calculate

$$
\begin{aligned}
\left|\int_{\mathbb{R} / l \mathbb{Z}}\left\langle\partial_{s}^{k+2}\left(P_{c^{\prime}}^{\perp} R^{\alpha}(c)\right), \partial_{s}^{k} \kappa\right\rangle d s\right| & =\left|\int_{\mathbb{R} / l \mathbb{Z}}\left\langle\partial_{s}^{k+1}\left(P_{c^{\prime}}^{\perp} R^{\alpha}(c)\right), \partial_{s}^{k+1} \kappa\right\rangle d s\right| \\
\leq & \left|\int_{\mathbb{R} / l \mathbb{Z}}\left\langle\partial_{s}^{k+1}\left(R^{\alpha}(c)\right), \partial_{s}^{k+1} \kappa\right\rangle d s\right| \\
& +\left|\int_{\mathbb{R} / l \mathbb{Z}}\left\langle\partial_{s}^{k+1}\left(\left\langle R^{\alpha}(c), c^{\prime}\right\rangle c^{\prime}\right), \partial_{s}^{k+1} \kappa\right\rangle d s\right|
\end{aligned}
$$

Using the definition of $R_{1}^{\alpha}$ and the Leibniz rule together with Lemma 3.6, we obtain 


$$
\begin{aligned}
& \left|\int_{\mathbb{R} / l \mathbb{Z}}\left\langle\partial_{s}^{k+1}\left(R_{1}^{\alpha}(c)\right), \partial_{s}^{k+1} \kappa\right\rangle d s\right| \\
& \leq C \sum_{i=1}^{k+1} \int_{\mathbb{R} / l \mathbb{Z}}\left|\partial_{s}^{i} \int_{-\frac{1}{2}}^{\frac{1}{2}}\left(\frac{1}{|c(s+w)-c(s)|^{\alpha}}-\frac{1}{|w|^{\alpha}}\right) d w\right| \mid \partial_{s}^{k+1-i} c^{\prime \prime} \| \partial_{s}^{k+1} c^{\prime \prime} d s \\
& \leq C \sum_{i=1}^{k+1} \int_{\mathbb{R} / l \mathbb{Z}}\left|\int_{-\frac{1}{2}}^{\frac{1}{2}} \partial_{s}^{i}\left(\frac{1}{|c(s+w)-c(s)|^{\alpha}}-\frac{1}{|w|^{\alpha}}\right) d w\right|\left|P_{2}^{2 k+2-i}\left(c^{\prime \prime}\right)\right| d s \\
& \leq C \sum_{i=1}^{k+1} \int_{\mathbb{R} / l \mathbb{Z}}\left|\int_{-\frac{1}{2}}^{\frac{1}{2}} \partial_{s}^{i}\left(\frac{1}{|c(s+w)-c(s)|^{\alpha}}-\frac{1}{|w|^{\alpha}}\right) d w\right|\left|P_{2}^{2 k+4-i}\left(c^{\prime}\right)\right| d s \\
& \leq \varepsilon\left\|D^{k+2+\frac{\alpha+1}{2}} c\right\|_{L^{2}}^{2}+C_{\varepsilon}
\end{aligned}
$$

since $(i+2 k+4-i) /(k+2)=(2 k+4) /(k+2)=2$. Along the same lines we get

$$
\begin{gathered}
\int_{\mathbb{R} / l \mathbb{Z}}\left|\left\langle\partial_{s}^{k+1}\left(R_{2}^{\alpha}(c)\right), \partial_{s}^{k+1} \kappa\right\rangle\right| d s \\
\leq \varepsilon\left\|D^{k+2+\frac{\alpha+1}{2}} c\right\|_{L^{2}}^{2}+C_{\varepsilon} .
\end{gathered}
$$

Furthermore, we can estimate

$$
\begin{aligned}
& \left|\int_{\mathbb{R} / l \mathbb{Z}}\left\langle\partial_{s}^{k+1}\left(\left\langle R^{\alpha}(c), c^{\prime}\right\rangle c^{\prime}\right), \partial_{s}^{k+1} \kappa\right\rangle d s\right| \\
& \quad \leq C \sum_{i+j+m=k+1}\left|\int_{\mathbb{R} / l \mathbb{Z}}\left\langle\left(\left\langle\partial^{i} R^{\alpha}(c), \partial^{j+1} c\right\rangle \partial^{m+1} c\right), \partial_{s}^{k+1} \kappa\right\rangle d s\right| \\
& \quad \leq C \sum_{i=0}^{k+1}\left|\int_{\mathbb{R} / l \mathbb{Z}} \partial^{i} R^{\alpha}(c) * P_{3}^{2 k+3-i}\left(c^{\prime}\right) d s\right| \\
& \leq \varepsilon\left\|D^{k+2+\frac{\alpha+1}{2}} c\right\|_{L^{2}}^{2}+C_{\varepsilon}
\end{aligned}
$$

Using the same line of arguments one sees that also the terms

$$
\left|\int_{\mathbb{R} / l \mathbb{Z}}\left\langle P_{2}^{k}\left(P_{c^{\prime}}^{\perp} R^{\alpha} c, \kappa\right) \tau, \partial_{s}^{k+1} \kappa\right\rangle d s\right|,\left|\int_{\mathbb{R} / l \mathbb{Z}}\left\langle P_{3}^{k}\left(P_{c^{\prime}}^{\perp}\left(R^{\alpha} c, \kappa\right), \partial_{s}^{k} \kappa\right)\right\rangle d s\right|
$$


and

$$
\left.\left|\int_{\mathbb{R} / l \mathbb{Z}}\right| \partial_{s}^{k} \kappa\right|^{2}\left\langle\kappa, P_{c^{\prime}}^{\perp} R^{\alpha} c\right\rangle d s \mid
$$

can be estimated by

$$
\varepsilon\left\|D^{k+2+\frac{\alpha+1}{2}} c\right\|_{L^{2}}^{2}+C_{\varepsilon} .
$$

For the terms containing $\lambda \kappa$ we use Hölder's inequality and standard GagliardoNirenberg-Sobolev estimates together with Cauchy's inequality as above, to estimate these terms by

$$
\varepsilon\left\|D^{k+2+\frac{\alpha+1}{2}} c\right\|_{L^{2}}^{2}+C(\varepsilon) .
$$

To estimate the terms containing $Q^{\alpha}$, we will use the fact that $Q^{\alpha}=c_{\alpha} D^{\alpha+1}+\tilde{R}$ where $\tilde{R}$ is a bounded operator from $W^{s+2, p}$ to $W^{s, p}$ [24, Proposition 2.3]. For $k_{1}, k_{2} \in$ $\mathbb{N}_{0}$ with $k_{1}+k_{2}=k$ we estimate using Hölder's inequality and the GagliardoNirenberg-Sobolev inequality

$$
\begin{aligned}
\left|\int_{\mathbb{R} / l \mathbb{Z}}\left\langle\left(\partial_{s}^{k_{1}} Q^{\alpha} c * \partial_{s}^{k_{2}} \kappa\right) \tau, \partial_{s}^{k+1} \kappa\right\rangle d s\right| & \leq C\left\|Q^{\alpha} \partial_{s}^{k_{1}} c\right\|_{L^{2}}\left\|\partial_{s}^{k_{2}} \kappa\right\|_{L^{4}}\left\|\partial_{s}^{k+1} \kappa\right\|_{L^{4}} \\
& \leq C\left\|\partial_{s}^{k_{1}} c\right\|_{W^{\alpha+1,2}}\left\|\partial_{s}^{k_{2}} \kappa\right\|_{L^{4}}\left\|\partial_{s}^{k+1} \kappa\right\|_{L^{4}} \\
& \leq \varepsilon\left\|D^{k+2+\frac{\alpha+1}{2}} c\right\|_{L^{2}}^{2}+C(\varepsilon)
\end{aligned}
$$

and thus

$$
\left|\int_{\mathbb{R} / l \mathbb{Z}}\left\langle P_{2}^{k}\left(Q^{\alpha} c, \kappa\right) \tau, \partial_{s}^{k+1} \kappa\right\rangle d s\right| \leq \varepsilon\left\|D^{k+2+\frac{\alpha+1}{2}} c\right\|_{L^{2}}^{2}+C(\varepsilon) .
$$

Similarly we obtain, using the Leibniz rule,

$$
\left|\int_{\mathbb{R} / l \mathbb{Z}}\left\langle\left(\partial_{s}^{k_{1}} P_{c^{\prime}}^{\perp} Q^{\alpha} c * \partial_{s}^{k_{2}} \kappa\right) \tau, \partial_{s}^{k+1} \kappa\right\rangle d s\right| \leq \varepsilon\left\|D^{k+2+\frac{\alpha+1}{2}} c\right\|_{L^{2}}^{2}+C_{\varepsilon} .
$$

Hence,

$$
\left|\int_{\mathbb{R} / l \mathbb{Z}}\left\langle P_{2}^{k}\left(P_{c^{\prime}}^{\perp} Q^{\alpha} c, \kappa\right) \tau, \partial_{s}^{k+1} \kappa\right\rangle d s\right| \leq \varepsilon\left\|D^{k+2+\frac{\alpha+1}{2}} c\right\|_{L^{2}}^{2}+C_{\varepsilon} .
$$

Similarly, one gets

$$
\begin{aligned}
& \left|\int_{\mathbb{R} / l \mathbb{Z}}\left\langle P_{3}^{k}\left(P_{c^{\prime}}^{\perp} Q^{\alpha} c, \kappa\right), \partial_{s}^{k} \kappa\right\rangle d s\right|+\left.\left|\int_{\mathbb{R} / l \mathbb{Z}}\right| \partial_{s}^{k} \kappa\right|^{2}\left\langle\kappa, P_{c^{\prime}}^{\perp} Q^{\alpha} c\right\rangle d s \mid \\
& \quad \leq \varepsilon\left\|D^{k+2+\frac{\alpha+1}{2}} c\right\|_{L^{2}}^{2}+C_{\varepsilon} .
\end{aligned}
$$


Let us finally turn to the term

$$
\begin{aligned}
\int_{\mathbb{R} / l \mathbb{Z}} & \left\langle\partial_{s}^{k+2}\left(P_{c^{\prime}}^{\perp} Q^{\alpha} c\right), \partial_{s}^{k} \kappa\right\rangle d s=\int_{\mathbb{R} / l \mathbb{Z}}\left\langle\partial_{s}^{k+2}\left(Q^{\alpha} c\right), \partial_{s}^{k} \kappa\right\rangle d s \\
& -\int_{\mathbb{R} / l \mathbb{Z}}\left\langle\partial_{s}^{k+2}\left(\left\langle Q^{\alpha} c, c^{\prime}\right\rangle c^{\prime}\right), \partial_{s}^{k} \kappa\right\rangle d s \\
= & \int_{\mathbb{R} / l \mathbb{Z}}\left\langle Q^{\alpha}\left(\partial^{k} \kappa\right), \partial_{s}^{k} \kappa\right\rangle d s-\int_{\mathbb{R} / l \mathbb{Z}}\left\langle\partial_{s}^{k+2}\left(\left\langle Q^{\alpha} c, c^{\prime}\right\rangle c^{\prime}\right), \partial_{s}^{k} \kappa\right\rangle d s \\
= & c_{\alpha} \int_{\mathbb{R} / l \mathbb{Z}}\left\langle D^{\alpha+1}\left(\partial^{k} \kappa\right), \partial_{s}^{k} \kappa\right\rangle d s+\int_{\mathbb{R} / l \mathbb{Z}}\left\langle\tilde{R}\left(\partial^{k} \kappa\right), \partial_{s}^{k} \kappa\right\rangle d s \\
& -\int_{\mathbb{R} / l \mathbb{Z}}\left\langle\partial_{s}^{k+2}\left(\left\langle Q^{\alpha} c, c^{\prime}\right\rangle c^{\prime}\right), \partial_{s}^{k} \kappa\right\rangle d s \\
= & c_{\alpha}\left\langle D^{\frac{\alpha+1}{2}}\left(\partial^{k} \kappa\right), D^{\frac{\alpha+1}{2}} \partial_{s}^{k} \kappa\right\rangle d s-\int_{\mathbb{R} / l \mathbb{Z}}\left\langle\tilde{R}\left(\partial^{k-1} \kappa\right), \partial_{s}^{k+1} \kappa\right\rangle d s \\
- & \int_{\mathbb{R} / l \mathbb{Z}}\left\langle\partial_{s}^{k+2}\left(\left\langle Q^{\alpha} c, c^{\prime}\right\rangle c^{\prime}\right), \partial_{s}^{k} \kappa\right\rangle d s
\end{aligned}
$$

Using Taylor's approximation we see that

$$
\begin{gathered}
Q^{\alpha} f(x)=p . v \cdot \int_{-\frac{l}{2}}^{\frac{l}{2}}\left(2 \frac{f(x+w)-f(x)-w f^{\prime}(x)}{w^{2}}-f^{\prime \prime}(x)\right) \frac{d w}{|w|^{\alpha}} \\
=p . v . \int_{-\frac{l}{2}}^{\frac{l}{2}} \frac{\int_{0}^{1} 2(1-\tau)\left(f^{\prime \prime}(x+\tau w)-f^{\prime \prime}(x)\right) d \tau}{|w|^{\alpha}} d w
\end{gathered}
$$

and hence

$$
\begin{aligned}
& \int_{\mathbb{R} / l \mathbb{Z}}\left\langle Q^{\alpha} f(x), g(x)\right\rangle d x \\
& =p \cdot v \cdot \int_{\mathbb{R} / l \mathbb{Z}} \int_{-\frac{l}{2}}^{\frac{l}{2}} \frac{\int_{0}^{1} 2(1-\tau)\left\langle f^{\prime \prime}(x+\tau w)-f^{\prime \prime}(x), g(x)\right\rangle d \tau}{|w|^{\alpha}} d w d x \\
& =-\int_{\mathbb{R} / l \mathbb{Z}} \int_{-\frac{l}{2}}^{\frac{l}{2}} \frac{\int_{0}^{1}(1-\tau)\left(\left\langle f^{\prime \prime}(x+\tau w)-f^{\prime \prime}(x), g(x+\tau w)-g(x)\right\rangle\right) d \tau}{|w|^{\alpha}} d w d x
\end{aligned}
$$


and substituting first $\tilde{x}=x+\tau w$ and then $\tilde{w}=-w$ shows

$$
\begin{aligned}
& \int_{\mathbb{R} / l \mathbb{Z}-\frac{l}{2}} \int_{\int_{0}}^{\frac{l}{2}} \frac{\int_{0}^{1}(1-\tau)\left\langle f^{\prime \prime}(x+\tau w)-f^{\prime \prime}(x), g(x)\right\rangle d \tau}{|w|^{\alpha}} d w d x \\
& =-\int_{\mathbb{R} / l \mathbb{Z}} \int_{-\frac{l}{2}}^{\frac{l}{2}} \frac{\int_{0}^{1}(1-\tau)\left\langle f^{\prime \prime}(\tilde{x}-\tau w)-f^{\prime \prime}(\tilde{x}), g(\tilde{x}-\tau w)\right\rangle d \tau}{|w|^{\alpha}} d w d \tilde{x} \\
& =-\int_{\mathbb{R} / l \mathbb{Z}} \int_{-\frac{l}{2}}^{\frac{l}{2}} \frac{\int_{0}^{1}(1-\tau)\left\langle f^{\prime \prime}(\tilde{x}+\tau \tilde{w})-f^{\prime \prime}(\tilde{x}), g(\tilde{x}+\tau \tilde{w})\right\rangle d \tau}{|\tilde{w}|^{\alpha}} d \tilde{w} d \tilde{x} .
\end{aligned}
$$

The Leibniz rule now tells us that

$$
\begin{aligned}
& \left|\int_{\mathbb{R} / l \mathbb{Z}}\left\langle\partial_{s}^{k+2}\left(\left\langle Q^{\alpha} c, c^{\prime}\right\rangle c^{\prime}\right), \partial_{s}^{k} \kappa\right\rangle d s\right| \\
& \quad \leq C \sum_{i+j+m=k+2}\left|\int_{\mathbb{R} / l \mathbb{Z}}\left\langle\left(\left\langle Q^{\alpha}\left(\partial^{i} c\right), \partial^{j+1} c\right\rangle \partial^{m+1} c\right), \partial_{s}^{k} \kappa\right\rangle d s\right| .
\end{aligned}
$$

Setting $g_{m, j}=\partial^{j+1} c\left\langle\partial^{m+1} c, \partial_{s}^{k} \kappa\right\rangle$ and using the formula above for $Q^{\alpha}$ we get

$$
\begin{aligned}
& \left|\int_{\mathbb{R} / l \mathbb{Z}}\left\langle\partial_{s}^{k+2}\left(\left\langle Q^{\alpha} c, c^{\prime}\right\rangle c^{\prime}\right), \partial_{s}^{k} \kappa\right\rangle d s\right| \leq C \sum_{i+j+m=k+2} \mid \int_{\mathbb{R} / l \mathbb{Z}} \int_{-\frac{l}{2}}^{\frac{l}{2}} \\
& \quad \times \frac{\int_{0}^{1}(1-\tau)\left(\left\langle\partial^{i+2} c(x+\tau w)-\partial^{i+2} c(x), g_{m, j}(x+\tau w)-g_{m, j}(x)\right\rangle\right) d \tau}{|w|^{\alpha}} d w d s \mid \\
& \leq C \sum_{i+j+m=k+2}\left|\partial^{i+2} c\right| W^{\frac{\alpha-1}{2}, 2}\left|g_{j, m}\right|_{W^{\frac{\alpha-1}{2}, 2}}
\end{aligned}
$$

Using the fractional Leibniz rule Lemma 8.2 and then the interpolation estimates, we can estimate this again by

$$
\varepsilon\left\|D^{k+2+\frac{\alpha+1}{2}} c\right\|_{L^{2}}+C(\varepsilon) .
$$

As furthermore

$$
\begin{aligned}
\int_{\mathbb{R} / l \mathbb{Z}}\left\langle\tilde{R}\left(\partial_{s}^{k-1} \kappa\right), \partial_{s}^{k+1} \kappa\right\rangle d s & \leq\left\|\partial_{s}^{k+1} \kappa\right\|_{L^{2}}\left\|\tilde{R}\left(\partial_{s}^{k-1} \kappa\right)\right\|_{L^{2}} \leq C\left\|\partial_{s}^{k+1} \kappa\right\|_{L^{2}}^{2} \\
& \leq \varepsilon\left\|D^{k+2+\frac{\alpha+1}{2}} c\right\|_{L^{2}}+C_{\varepsilon}
\end{aligned}
$$


we finally get

$$
\int_{\mathbb{R} / \mathbb{Z}}\left\langle\partial_{s}^{k+2}\left(P_{c^{\prime}}^{\perp} Q^{\alpha} c\right), \partial_{s}^{k} \kappa\right\rangle d s \leq-c_{\alpha} \int_{\mathbb{R} / l \mathbb{Z}}\left|D_{s}^{k+\frac{\alpha+1}{2}} \kappa\right|^{2} d s+\varepsilon \int_{\mathbb{R} / l \mathbb{Z}}\left|D_{s}^{k+\frac{\alpha+1}{2}} \kappa\right|^{2} d s+C_{\varepsilon} .
$$

Summing up these estimates proves Lemma 3.7.

A standard argument now concludes the proof of Theorem 3.1:

Proof Theorem 3.1 Let us assume that $[0, T)$ is the maximal interval of existence and $T<\infty$. If we apply Lemma 3.7 with $\varepsilon=\frac{c_{\alpha}}{2}$ we get

$$
\frac{d}{d t} \mathcal{E}^{k}+\frac{c_{\alpha}}{2}\left\|D^{k+2+\frac{\alpha+1}{2}} c\right\|_{L^{2}}^{2} \leq C_{k}
$$

Together with the Poincare inequality

$$
\mathcal{E}^{k} \leq C\left\|D^{k+2+\frac{\alpha+1}{2}} c\right\|_{L^{2}}^{2},
$$

this yields

$$
\frac{d}{d t} \mathcal{E}^{k}+c \mathcal{E}^{k} \leq C_{k}
$$

for a constant $c>0$. Hence,

$$
\left\|\partial_{s}^{k} \kappa_{c_{t}}\right\|_{L^{2}}^{2} \leq C(k)
$$

for a constant $C(k)<\infty$ depending on the inital data but not on $T$. Furthermore,

$$
\partial_{t}\left|c^{\prime}\right|=-\langle V, \kappa\rangle\left|c^{\prime}\right|
$$

which can be rewritten as

$$
\frac{d}{d t} \log \left(|c|^{\prime}\right)=-\langle V, \kappa\rangle
$$

Since $\langle V, \kappa\rangle$ is uniformly bounded there is a constant $C>0$ depending on $T$ such that

$$
C^{-1} \leq\left|c^{\prime}\right| \leq C
$$

It was shown in [12, end of p.7], that this estimate together with the uniform in time bounds on all derivatives with respect to arc-length yield

$$
\left\|\partial_{x}^{k} c\right\| \leq C_{k}
$$

for constants $C_{k}$ that are independent of $t$. 
Thus, there is a subsequence $t_{i} \rightarrow T$ such that $c_{t_{i}}$ converges smoothly to a $\tilde{c}(T)$ and we can use the short time existence result Theorem 2.1 to extend the flow beyond $T$.

Let us finally prove the subconvergence to a critical point up to translations and reparametrizations. Let the re-parametrizsations $\psi_{t}$ be such that the curves

$$
\begin{aligned}
\tilde{c}_{t} & : \mathbb{R} / \mathbb{Z} \rightarrow \mathbb{R}^{n} \\
& \mapsto \mapsto c_{t}\left(\psi_{t}(s)\right)-c_{t}(0)
\end{aligned}
$$

are parametrized proportional by arc-length. As the uniform in time estimates (3.13) hold, there is a subsequence $t_{i} \rightarrow \infty$ such that the curves $\tilde{c}_{t_{i}}$ converge smoothly to a curve $c_{\infty}: \mathbb{R} / \mathbb{Z} \rightarrow \mathbb{R}^{n}$. Since

$$
\int_{0}^{\infty}\left(\int_{\mathbb{R} / \mathbb{Z}}\left|\partial_{t} \tilde{c}_{t}\right|^{2} d s\right) d t \leq E^{\alpha}\left(c_{0}\right)+\lambda L\left(c_{0}\right)<\infty
$$

we get

$$
\int_{t_{i}}^{t_{i}+1}\left(\int_{\mathbb{R} / \mathbb{Z}}\left|\mathfrak{V}^{\alpha}\left(\tilde{c}_{t}\right)+\lambda \kappa_{\tilde{c}_{t}}\right|^{2} d s\right) d t=\int_{t_{i}}^{t_{i}+1}\left(\int_{\mathbb{R} / \mathbb{Z}}\left|\partial_{t} \tilde{c}_{t}\right|^{2} d s\right) d t \stackrel{i \rightarrow \infty}{\longrightarrow} 0 .
$$

Since our uniform estimates further imply

$$
\left|\frac{d}{d t} \int_{\mathbb{R} / \mathbb{Z}}\right| \mathfrak{V}^{\alpha}\left(\tilde{c}_{t}\right)+\left.\lambda \kappa_{\tilde{c}_{t}}\right|^{2} d s \mid \leq C
$$

for a constant $C<\infty$ independent of time, we deduce

$$
\int_{\mathbb{R} / \mathbb{Z}}\left|\mathfrak{V}^{\alpha}\left(\tilde{c}_{t}\right)+\lambda \kappa_{\tilde{c}_{t}}\right|^{2} d s \stackrel{t \rightarrow \infty}{\longrightarrow} 0
$$

which shows that $c_{\infty}$ is a critical point of $E^{\alpha}+\lambda L$.

\section{Asymptotics of the flows}

\section{1 Łojasiewicz-Simon gradient estimate}

In order to prove convergence to critical points of the complete flow without taking care of translations, we will prove a Łojawiewicz-Simon gradient estimate. 
Theorem 4.1 (Łojasiewicz-Simon gradient Estimate) Let $c_{M}$ be a smooth critical point of $E^{\alpha}+\lambda L$ for some $\alpha \in(2,3)$ and $\lambda>0$. Then there are constants $\theta \in[0,1 / 2]$, $\sigma>0, C<\infty$, such that every $c \in H_{i, r}^{\alpha+1}\left(\mathbb{R} / \mathbb{Z}, \mathbb{R}^{n}\right)$ with $\left\|c-c_{M}\right\|_{H^{\alpha+1}} \leq \sigma$ satisfies

$$
\left|E(c)-E\left(c_{M}\right)\right|^{1-\theta} \leq C\left(\int_{\mathbb{R} / \mathbb{Z}}|(V c)(x)|^{2}\left|c^{\prime}(x)\right| d x\right)^{1 / 2},
$$

where $V c=-\mathfrak{V}^{\alpha} c+\lambda \kappa_{c}$.

Proof After scaling the curve we can assume that $c_{M}$ is parameterized by arc length and that the length of the curve $c_{M}$ is 1 .

Let $H^{\alpha+1}\left(\mathbb{R} / \mathbb{Z}, \mathbb{R}^{n}\right)_{c_{M}}^{\perp}$ denote the space of all vector fields $N \in H^{\alpha+1}\left(\mathbb{R} / \mathbb{Z}, \mathbb{R}^{n}\right)$ which are orthogonal to $c_{M}^{\prime}$.

We will first prove that there are constants $\theta \in[0,1 / 2], \tilde{\sigma}>0$, and $C<\infty$, such that

$$
\left|E\left(c_{M}+N\right)-E\left(c_{M}\right)\right|^{1-\theta} \leq C\left(\int_{\mathbb{R} / \mathbb{Z}}\left|V\left(c_{M}+N\right)\right|^{2} d x\right)^{1 / 2}
$$

for all $N \in H^{\alpha+1}\left(\mathbb{R} / \mathbb{Z}, \mathbb{R}^{n}\right)_{c_{M}}^{\perp}$ with $\|N\|_{H^{\alpha+1}} \leq \tilde{\sigma}$. That is, we show that the functional

$$
\begin{array}{r}
\tilde{E}: H^{\alpha+1}\left(\mathbb{R} / \mathbb{Z}, \mathbb{R}^{n}\right)_{c_{M}}^{\perp} \rightarrow \mathbb{R} \\
N \mapsto E^{\alpha}\left(c_{M}+N\right)+\lambda L\left(c_{M}+N\right)
\end{array}
$$

satisfies a Łojasiewicz-Simon gradient estimate. By [10, Corollary 3.11] it suffices to show that $E^{\prime}$ is analytic with values in $L^{2}$ and that $E^{\prime \prime}$ is a Fredholm operator from $H^{\alpha+1}\left(\mathbb{R} / \mathbb{Z}, \mathbb{R}^{n}\right)_{c_{M}}^{\perp}$ to $L^{2}\left(\mathbb{R} / \mathbb{Z}, \mathbb{R}^{n}\right)$.

It is easy to see that $\kappa$ defines an analytic operator on a neighborhood of 0 from $\left(H^{\alpha+1}\right)_{c_{M}}^{\perp}$ to $L^{2}$ using the fact that $H^{\alpha+1}$ is embedded in $C^{2}$. That the same is true for $\mathfrak{V}^{\alpha}$ can be seen from Theorem 2.3 , using the fact that $H^{\alpha+1}\left(\mathbb{R} / \mathbb{Z}, \mathbb{R}^{n}\right)$ is embedded in $C^{\alpha+\beta}$ for every $0<\beta<1 / 2$ and $C^{\beta}$ is embedded in $L^{2}$.

We calculate the second variation of $\tilde{E}$ at 0 and try to write it as a compact perturbation of a Fredholm operator of index 0 . Using $\left|c_{M}^{\prime}\right|=1$ and the fact that $V$ is the gradient of $E^{\alpha}+\lambda L$, we get

$$
\begin{aligned}
& \tilde{E}^{\prime \prime}(0)\left(h_{1}, h_{2}\right) \\
& =\lim _{t \rightarrow 0} \frac{\int_{\mathbb{R} / \mathbb{Z}}\left\langle V\left(c_{M}+t h_{1}\right), h_{2}\right\rangle\left|c_{M}^{\prime}+t h_{1}^{\prime}\right| d w-\int_{\mathbb{R} / \mathbb{Z}}\left\langle V\left(c_{M}\right), h_{2}\right\rangle\left|c_{M}^{\prime}\right| d w}{t} \\
& =\left\langle\delta_{h_{1}} V c_{M}, h_{2}\right\rangle_{L^{2}}+\left\langle L_{1} h_{1}, h_{2}\right\rangle_{L^{2}}
\end{aligned}
$$

where $L_{1} h_{1}=\mathfrak{V}^{\alpha} c_{M} \cdot\left\langle c_{M}^{\prime}, h_{1}^{\prime}\right\rangle$ is a differential operator of order 1 in $h_{1}$.

We know from Theorem 2.3 that

$$
V c=\mathfrak{V}^{\alpha} c+\lambda \kappa_{c}=\frac{\alpha}{\left|c^{\prime}\right|^{\alpha+1}} P_{c^{\prime}}^{\perp}\left(Q^{\alpha} c\right)+F^{\alpha}(c)
$$


where $F^{\alpha} \in C^{\omega}\left(C^{\alpha+\beta}, C^{\beta}\right)$ for all $\beta>0$. Thus

$$
P_{c^{\prime}}^{\perp}\left(\delta_{h} V\left(c_{M}\right)\right)=\frac{\alpha}{\left|c^{\prime}\right|^{\alpha+1}}\left(P_{c^{\prime}}^{\perp}\left(Q^{\alpha} h\right)+L_{2}(h)\right)
$$

where

$$
\begin{aligned}
L_{2}(h) & =-\frac{\alpha(\alpha+1)}{\left|c^{\prime}\right|^{\alpha+3}}\left\langle c_{M}^{\prime}, h^{\prime}\right\rangle P_{c^{\prime}}^{\perp}\left(Q^{\alpha} c_{M}\right)+P_{c^{\prime}}^{\perp}\left(\delta_{h} F^{\alpha}\left(c_{M}\right)+\alpha\left(\delta_{h} P_{c^{\prime}}^{\perp}\right)\left(Q^{\alpha} c_{M}\right)\right) \\
& \in C^{\omega}\left(C^{\alpha+\beta}, C^{\beta}\right) \quad \forall \beta>0 .
\end{aligned}
$$

Now let $v_{i}, i=1,2, \ldots,(n-1)$, be smooth functions such that $v_{1}(u), \ldots, v_{n-1}(u)$ is an orthonormal basis of the normal space on $c_{M}$ at $u$. Then each $\phi \in$ $H^{\alpha+1}\left(\mathbb{R} / \mathbb{Z}, \mathbb{R}^{n}\right)_{c_{M}}^{\perp}$ can be written in the form

$$
\phi=\sum_{i=1}^{n-1} \phi_{i} v_{i}
$$

where $\phi_{i}:=\left\langle\phi, v_{i}\right\rangle \in H^{\alpha+1}(\mathbb{R} / \mathbb{Z})$. We calculate

$$
\begin{aligned}
& P_{c^{\prime}}^{\perp}\left(Q^{\alpha} \phi\right) \\
& =P_{c^{\prime}}^{\perp}\left(Q^{\alpha}\left(\sum_{i=1}^{n-1} \phi_{i} v_{i}\right)\right)=\sum_{i=1}^{n-1}\left(Q^{\alpha} \phi_{i}\right) P_{c^{\prime}}^{\perp}\left(v_{i}\right)+P_{c^{\prime}}^{\perp}\left(Q^{\alpha}\left(\phi_{i} v_{i}\right)-\left(Q^{\alpha} \phi_{i}\right) v_{i}\right) \\
& =\sum_{i=1}^{n-1}\left(Q^{\alpha} \phi_{i}\right) P_{c^{\prime}}^{\perp} v_{i}+L_{3}(\phi)
\end{aligned}
$$

where $L_{3} \in C^{\omega}\left(C^{\alpha+\beta}, C^{\beta}\right)$ by the fractional Leibniz rule Lemma 7.1.

From [24, Proposition 2.3] we know that there is a constant $a^{(\alpha)}>0$ such that that $L_{4}:=Q^{\alpha}-a^{\alpha}(-\Delta)^{(\alpha+1) / 2}$ is a bounded linear operator from $H^{2}$ to $L^{2}$. Combining (4.3), (4.4), and (4.5), we get

$$
E^{\prime \prime}(0)\left(h_{1}, h_{2}\right)=\left\langle\sum_{i=1}^{n-1} a^{\alpha}\left(\left(-\Delta^{\frac{\alpha+1}{2}}\right)\left\langle h_{1}, v_{i}\right\rangle\right) v_{i}+L h_{1}, h_{2}\right\rangle
$$

where

$$
L:=L_{1}+L_{2}+L_{3}+L_{4}
$$

is a bounded operator from $C^{\alpha+\varepsilon}$ to $L^{2}$ for all $\varepsilon>0$.

Since the linear mapping

$$
h_{1} \rightarrow\left(\begin{array}{c}
\left\langle h_{1}, v_{1}\right\rangle \\
\vdots \\
\left\langle h_{1}, v_{n-1}\right\rangle
\end{array}\right)
$$


defines an homeomorphism between $H^{s}\left(\mathbb{R} / \mathbb{Z}, \mathbb{R}^{n}\right)_{c_{M}}^{\perp}$ and $H^{s}\left(\mathbb{R} / \mathbb{Z}, \mathbb{R}^{n-1}\right)$ for all $s \geq 0$ and $(-\Delta)^{(\alpha+1) / 2}$ is a Fredholm operator of index zero from $H^{\alpha+1}\left(\mathbb{R} / \mathbb{Z}, \mathbb{R}^{n-1}\right)$ to $L^{2}\left(\mathbb{R} / \mathbb{Z}, \mathbb{R}^{n-1}\right)$, the operator

$$
\begin{aligned}
A: H^{\alpha+1}\left(\mathbb{R} / \mathbb{Z}, \mathbb{R}^{n}\right)_{c_{M}}^{\perp} & \rightarrow L^{2}\left(\mathbb{R} / \mathbb{Z}, \mathbb{R}^{n}\right)_{c_{M}}^{\perp} \\
\phi & \mapsto \sum_{i=1}^{n-1}\left((-\Delta)^{\frac{\alpha+1}{2}}\left\langle\phi, v_{i}\right\rangle\right) v_{i}
\end{aligned}
$$

is Fredholm of order 0. Hence, $\sum_{i=1}^{n-1} a^{(\alpha)}\left(-\Delta^{\frac{\alpha+1}{2}}\right)\left\langle h_{1}, v_{i}\right\rangle v_{i}+L h_{1}$ as a compact perturbation of $A$ is a Fredholm operator as well. This implies that $E^{\prime \prime}$ is a Fredholm operator from $H^{\alpha+1}\left(\mathbb{R} / \mathbb{Z}, \mathbb{R}^{n}\right)_{c_{M}}^{\perp}$ to $L^{2}\left(\mathbb{R} / \mathbb{Z}, \mathbb{R}^{n}\right)_{c_{M}}^{\perp}$ of index 0 . The proof of (4.2) is complete.

To prove the full estimate of Theorem 4.1, we use Lemma 9.1 to write curves close to $c_{M}$ as normal graphs over $c_{M}$. More precisely, we can choose $\sigma \in(0, \tilde{\sigma})$ such that for all $c \in H^{\alpha+1}(\mathbb{R} / \mathbb{Z})$ with $\left\|c-c_{M}\right\|_{H^{\alpha+1}} \leq \sigma$ there is a re-parameterization $\psi \in H^{\alpha+1}(\mathbb{R} / \mathbb{Z}, \mathbb{R} / \mathbb{Z})$ and an $N_{c} \in H^{\alpha+1}\left(\mathbb{R} / \mathbb{Z}, \mathbb{R}^{n}\right)^{\perp}$ such that

$$
c \circ \psi=c_{M}+N_{c}
$$

and

$$
\left\|N_{c}\right\|_{H^{\alpha+1}} \leq C \cdot\left\|c-c_{M}\right\|_{H^{\alpha+1}} .
$$

Making $\sigma>0$ smaller if necessary, using that $c_{M}$ is parameterized by arc length and that $H^{\alpha+1}$ is embedded continuously in $C^{1}$, we furthermore can achieve that

$$
\left|c^{\prime}\right|>\left|c_{M}^{\prime}\right|-\left|c_{M}^{\prime}-c^{\prime}\right| \geq \frac{1}{2}
$$

Thus,

$$
\begin{aligned}
\left|E(c)-E\left(c_{M}\right)\right|^{1-\theta} & =\left|E\left(c_{M}+N_{c}\right)-E\left(c_{M}\right)\right|^{1-\theta} \\
& \leq C\left(\int_{\mathbb{R} / \mathbb{Z}}\left|V\left(c_{M}+N_{c}\right)(x)\right|^{2} d x\right)^{1 / 2} \\
& \leq C \sqrt{2}\left(\int_{\mathbb{R} / \mathbb{Z}}\left|V\left(c_{M}+N_{c}\right)(x)\right|^{2}\left|c^{\prime}(x)\right| d x\right)^{1 / 2}
\end{aligned}
$$




\subsection{The flow above critical points}

In this section we apply the Łojasiewich-Simon gradient estimate to reprove longtime existence for solutions that approach a critical point from above as stated in Theorem 1.2. Using the techniques from this section, we will show that even the complete flow converges to a critical point without applying any translations.

Theorem 1.2 will follow easily from the following long time existence result for normal graphs over a critical point of the energies $E=E^{\alpha}+\lambda L$ :

Theorem 4.2 (Long time existence and asymptotics for normal graphs) Let $c_{M} \in$ $C^{\infty}\left(\mathbb{R} / \mathbb{Z}, \mathbb{R}^{n}\right)$ be a critical point of $E$ and let $k \in \mathbb{N}, \delta>0$ and $\beta>\alpha$. Then there is an open neighborhood $U^{\prime}$ of 0 in $\left(C^{\beta}\right)^{\perp}$ such that the following holds:

Suppose that $N \in C\left([0, T), h^{\beta}\left(\mathbb{R} / \mathbb{Z}, \mathbb{R}^{n}\right)_{c_{M}}^{\perp}\right) \cap C^{1}\left((0, T), C^{\infty}\left(\mathbb{R} / \mathbb{Z}, \mathbb{R}^{n}\right)_{c_{M}}^{\perp}\right)$ is a maximal solution of the equation

$$
\partial_{t}^{\perp}\left(c_{M}+N_{t}\right)=-\mathfrak{V}^{\alpha}\left(c_{M}+N_{t}\right)+\lambda \kappa
$$

with

$$
N_{0} \in U^{\prime}
$$

and

$$
E\left(c_{t}\right) \geq E\left(c_{M}\right)
$$

whenever $\left\|N_{t}\right\|_{C^{k}} \leq \delta$

Then $T=\infty$ and $N_{t}$ converges smoothly to an $N_{\infty} \in C^{\infty}\left(\mathbb{R} / \mathbb{Z}, \mathbb{R}^{n}\right)_{c_{M}}^{\perp}$ satisfying

$$
E\left(c_{M}+N_{\infty}\right)=E\left(c_{M}\right) .
$$

Furthermore $c_{M}+N_{\infty}$ is a critical point of the energy.

Proof Let $\tilde{k}=\max \{4, k,\lceil\beta+1\rceil\}$, where $\lceil\beta+1\rceil$ denotes the smallest integer larger or equal to $\beta+1$. Then of course we still have

$$
E\left(c_{t}\right) \geq E\left(c_{M}\right)
$$

under the stronger condition $\left\|N_{t}\right\|_{C^{\tilde{k}}} \leq \delta$.

We first use Theorem 4.1 to get an $\varepsilon_{0}>0$, a $\theta \in\left(0, \frac{1}{2}\right)$, and a constant $C<\infty$ such that

$$
\left|E\left(c_{M}+N\right)-E\left(c_{M}\right)\right|^{1-\theta} \leq C\left(\int_{\mathbb{R} / \mathbb{Z}}\left|V\left(c_{M}+N\right)\right|^{2} d x\right)^{1 / 2}
$$

for all $N \in C^{\tilde{k}}\left(\mathbb{R} / \mathbb{Z}, \mathbb{R}^{n}\right)_{c_{M}}^{\perp}$ with $\|N\|_{C^{\tilde{k}}} \leq \varepsilon_{0}$. 
Making $\varepsilon_{0}>0$ smaller if necessary, we can furthermore achieve that $\|N\|_{C^{1}} \leq \frac{1}{2}$ for all $N \in H^{\alpha+1}\left(\mathbb{R} / \mathbb{Z}, \mathbb{R}^{n}\right)_{c_{M}}^{\perp}$ with $\|N\|_{H^{\alpha+1}} \leq \delta$ and hence

$$
\left\|P_{c_{M}^{\prime}}^{\perp}-P_{c_{M}^{\prime}+N^{\prime}}^{\perp}\right\| \leq \frac{1}{2}
$$

and

$$
\left|c_{M}^{\prime}+N^{\prime}\right| \geq \frac{1}{2} \inf \left|c_{M}^{\prime}\right|
$$

on $\mathbb{R} / \mathbb{Z}$.

It is now crucial to use the smoothing properties of our short time existence result Theorem 2.5. This theorem tells us that there is a radius $r>0$ and a time $T>0$ such that the solution $N_{t}=N_{N_{0}, t}$ to

$$
\partial_{t}^{\perp}\left(c_{M}+N_{t}\right)=H\left(c_{M}+N_{t}\right)+\lambda \kappa_{c_{M}+N_{t}}
$$

with initial data $N_{0} \in U=\left\{N \in h^{\beta}\left(\mathbb{R} / \mathbb{Z}, \mathbb{R}^{n}\right)_{c_{M}}^{\perp}:\|N\|_{h^{\beta}} \leq r\right\}$ exists for $t \in[0, T)$. Furthermore, these solutions exist as long as they stay inside of $U$ as one can then always continue the solution.

Since the mapping

$$
\left(N_{0}, t\right) \rightarrow N_{N_{0}, t}
$$

belongs to $C^{\infty}\left(U \times(0, T), C^{\infty}(\mathbb{R} / \mathbb{Z})\right)$ and hence is especially continuous and that $N_{0, t}=0$, by making $r$ smaller if necessary we can guarantue that

$$
\left\|N_{t}\right\|_{C^{\tilde{k}}} \leq \varepsilon_{0}
$$

for all $t \in[T / 4, T / 2]$ and all initial data $N_{0} \in U$.

Let $\varepsilon \in\left(0, \min \left\{r, \varepsilon_{0}\right\}\right)$ to be chosen later. Using again that the mapping in Eq. (4.10) is continuous together with $N_{0, t}=0$ one sees that there is a $\delta \in(0, r)$ such that for $N_{0} \in U$ with $\left\|N_{0}\right\|_{h^{\beta}} \leq \delta$ we have

$$
\left\|N_{t}\right\|_{C^{\beta}} \leq \varepsilon \quad \forall t \in[T / 4, T / 2] .
$$

Now let $N \in C\left(\left[0, T_{\max }\right), h^{\beta}\left(\mathbb{R} / \mathbb{Z}, \mathbb{R}^{n}\right)_{c_{M}}^{\perp}\right) \cap C^{\infty}\left(\left(0, T_{\max }\right), C^{\infty}\left(\mathbb{R} / \mathbb{Z}, \mathbb{R}^{n}\right)_{c_{M}}^{\perp}\right)$ be a maximal solution of the equation

$$
\partial_{t}^{\perp}\left(c_{M}+N_{t}\right)=\mathfrak{V}^{\alpha}\left(c_{M}+N_{t}\right)+\lambda \kappa_{c_{M}+N_{t}}
$$

with $\left\|N_{0}\right\|_{h^{\beta}} \leq \delta$. We set $\tilde{c}_{t}=c_{M}+N_{t}$.

We know from the considerations above that $T_{\max } \geq T$,

$$
\left\|N_{\frac{T}{4}}\right\|_{C^{\beta}}<r
$$


and the solution exists at least as long as $\left\|N_{\frac{T}{4}}\right\|_{C^{\beta}}<r$. If the solution does not exist for all time, there hence is a $t_{0}>\frac{T}{2}$ such that

$$
\left\|N_{t}\right\|_{C^{\beta}}<r, \quad \forall t \in\left[\frac{T}{4}, t_{0}\right) .
$$

but

$$
\left\|N_{t_{0}}\right\|_{C^{\beta}}=r
$$

which leads to a contradiction for $\varepsilon>0$ small enough.

We will now show that

$$
\left\|N_{t}\right\|_{C^{\beta}} \leq C \varepsilon^{\theta \sigma}
$$

where $\sigma=\frac{\tilde{k}-\beta}{\tilde{k}+\frac{1}{2}}$.

Applying (4.11) for all times $t \in\left[\frac{T}{4}, t_{0}\right]$ we get

$$
\left\|N_{t}\right\|_{C^{\tilde{k}}} \leq \varepsilon, \quad \forall t \in\left[\frac{T}{2}, t_{0}\right]
$$

and hence $N_{t}$ satisfies a Łojasiewich-Simon gradient estimate (4.7) and (4.8) and (4.9) for all $t \in\left[\frac{T}{2}, t_{0}\right]$.

We calculate

$$
\begin{aligned}
\frac{d}{d t} E\left(\tilde{c}_{t}\right) & =-\int_{\mathbb{R} / \mathbb{Z}}\left\langle\partial_{t}^{\perp} \tilde{c}_{t}, V\left(\tilde{c}_{t}\right)\right\rangle\left|\tilde{c}_{t}^{\prime}\right| d x \\
& =-\int_{\mathbb{R} / \mathbb{Z}}\left|\partial_{t}^{\perp} \tilde{c}_{t}\right|^{2}\left|\tilde{c}_{t}^{\prime}\right| d x \\
& =-\int_{\mathbb{R} / \mathbb{Z}}\left|V \tilde{c}_{t}\right|^{2}\left|\tilde{c}_{t}^{\prime}\right| d x
\end{aligned}
$$

and hence

$$
\begin{aligned}
-\frac{d}{d t}\left(E\left(\tilde{c}_{t}\right)-E\left(c_{M}\right)\right)^{\theta} & =-\theta\left(E\left(\tilde{c}_{t}\right)-E\left(c_{M}\right)\right)^{\theta-1} \frac{d}{d t} E\left(\tilde{c}_{t}\right) \\
& \geq \frac{\theta}{C}\left(\int_{\mathbb{R} / \mathbb{Z}}\left|\partial_{t}^{\perp} \tilde{c}_{t}\right|^{2}\left|\tilde{c}_{t}^{\prime}\right| d x\right)^{1 / 2} \\
& \geq c\left(\int_{\mathbb{R} / \mathbb{Z}}\left|\partial_{t}^{\perp} \tilde{c}_{t}\right|^{2} d x\right)^{1 / 2}
\end{aligned}
$$


for a constant $c>0$. Integrating the above inequality over $\left(\frac{T}{2}, t\right)$ yields

$$
\begin{aligned}
\int_{\frac{T}{2}}^{t}\left(\int_{\mathbb{R} / \mathbb{Z}}\left|\partial_{\tau}^{\perp} \tilde{c}_{\tau}\right|^{2} d s\right)^{1 / 2} d \tau & \left.\leq C\left(\left(E\left(\tilde{c}_{\frac{T}{2}}\right)\right)-E\left(c_{M}\right)\right)^{\theta}-\left(E\left(\tilde{c}_{t}\right)-E\left(c_{M}\right)\right)^{\theta}\right) \\
& \left.\leq C\left(E\left(\tilde{c}_{\frac{T}{2}}\right)\right)-E\left(c_{M}\right)\right)^{\theta} .
\end{aligned}
$$

By (4.8) we have

$$
\left|\partial_{t} N_{t}\right|=\left|P_{\tilde{c}^{\prime}}^{\perp} \partial_{t} N_{t}\right| \leq\left|P_{\tilde{c}^{\prime}}^{\perp} \partial_{t} N_{t}\right|+\left|\left(P_{c^{\prime}}^{\perp}-P_{\tilde{c}^{\prime}}^{\perp}\right) \partial_{t} N_{t}\right| \leq\left|\partial_{t}^{\perp} \tilde{c}\right|+\frac{1}{2}\left|\partial_{t} N_{t}\right|
$$

Thus $\left|\partial_{t} \tilde{c}_{t}\right|=\left|\partial_{t} N_{t}\right| \leq 2\left|\partial_{t}^{\perp} \tilde{c}_{t}\right|$. Plugging this and (4.9) into the above inequality, we deduce

$$
\left.\int_{\frac{T}{2}}^{t}\left(\int_{\mathbb{R} / \mathbb{Z}}\left|\partial_{\tau} \tilde{c}_{\tau}\right|^{2} d x\right)^{1 / 2} \leq C\left(E\left(\tilde{c}_{\frac{T}{2}}\right)\right)-E\left(c_{M}\right)\right)^{\theta} .
$$

Hence,

$$
\begin{aligned}
\left\|\tilde{c}_{t}-c_{M}\right\|_{L^{2}(\mathbb{R} / \mathbb{Z}, d x)} & \leq\left\|\tilde{c}_{\frac{T}{2}}-\tilde{c}_{M}\right\|_{L^{2}(\mathbb{R} / \mathbb{Z}, d x)}+\left\|\tilde{c}_{\frac{T}{2}}-\tilde{c}_{t}\right\|_{L^{2}(\mathbb{R} / \mathbb{Z}, d x)} \\
& =\left\|\tilde{c}_{\frac{T}{2}}-c_{M}\right\|_{L^{2}(\mathbb{R} / \mathbb{Z}, d x)}+\left\{\int_{\mathbb{R} / l \mathbb{Z}}\left|\int_{\frac{T}{2}}^{t} \partial_{\tau} \tilde{c} d \tau\right|^{2} d x\right\}^{\frac{1}{2}} \\
& \leq\left\|\tilde{c}_{\frac{T}{2}}-c_{M}\right\|_{L^{2}(\mathbb{R} / \mathbb{Z}, d x)}+\int_{\frac{T}{2}}^{t}\left(\int_{\mathbb{R} / \mathbb{Z}}\left|\partial_{\tau} \tilde{c}\right|^{2} d s\right)^{\frac{1}{2}} d \tau \\
& \leq\left\|\tilde{c}_{\frac{T}{2}}-c_{M}\right\|_{L^{2}(\mathbb{R} / \mathbb{Z}, d x)}+C\left(E\left(\tilde{c}_{\frac{T}{2}}\right)-E\left(c_{M}\right)\right)^{\theta} \\
& \leq\left\|\tilde{c}_{\frac{T}{2}}-c_{M}\right\|_{L^{2}(\mathbb{R} / \mathbb{Z}, d x)}+C\left(E\left(\tilde{c}_{\frac{T}{2}}\right)-E\left(c_{M}\right)\right)^{\theta} \\
& \leq C\left\|\tilde{c}_{\frac{T}{2}}-c_{M}\right\|_{C^{\beta}}^{\theta} .
\end{aligned}
$$

Using the interpolation inequality

$$
\|f\|_{C^{\beta}} \leq\|f\|_{C^{\tilde{k}}}^{(1-\sigma)}\|f\|_{L^{2}(\mathbb{R} / \mathbb{Z}, d x)}^{\sigma}
$$


where $\sigma=\frac{\tilde{k}-\beta}{\tilde{k}+\frac{1}{2}}$ (cf. Lemma 8.3), we get for $t \in\left[\frac{T}{2}, t_{0}\right]$

$$
\begin{aligned}
& \left\|\tilde{c}_{t}-c_{M}\right\|_{C^{\beta}\left(\mathbb{R} / \mathbb{Z}, \mathbb{R}^{n}\right)} \\
& \quad \leq C\left\|\tilde{c}_{t}-c_{M}\right\|_{C^{\tilde{k}}\left(\mathbb{R} / \mathbb{Z}, \mathbb{R}^{n}\right)}^{1-\sigma}\left\|\tilde{c}_{t}-c_{M}\right\|_{L^{2}(\mathbb{R} / \mathbb{Z}, d x)}^{\sigma} \leq C\left\|\tilde{c}_{\frac{T}{2}}-c_{M}\right\|_{C^{\beta}}^{\theta \sigma} \\
& \quad \stackrel{(4.12)}{\leq} C \varepsilon^{\theta \sigma} .
\end{aligned}
$$

So if $\varepsilon>0$ is small enough we have

$$
\|N(t)\|_{C^{\beta}}<\frac{r}{4}
$$

for all $t \in\left[T, t_{0}\right]$ which contradicts our choice of $t_{0}$. Hence, we have shown that the flow exists for all time and satisfies

$$
\|N(t)\|_{C^{\beta}}<\frac{r}{4}
$$

for all $t \geq T$.

From Theorem 2.5 we get $\sup _{t \geq \frac{T}{2}}\left\|\tilde{c}_{t}\right\|_{C^{l}}<\infty$ for all $l \in \mathbb{N}$ and hence there is a subsequence $t_{i} \rightarrow \infty$ such that

$$
c_{t_{i}} \rightarrow c_{\infty}
$$

smoothly.

Since

$$
\int_{0}^{\infty}\left(\int_{\mathbb{R} / \mathbb{Z}}\left|\partial_{t} c_{t}\right|^{2} d x\right) d t \leq C \int_{0}^{\infty}\left(\int_{\mathbb{R} / l \mathbb{Z}}\left|\partial_{t} c_{t}\right|^{2} d s\right) d t \leq C E^{\alpha}\left(c_{0}\right)+C \lambda L\left(c_{0}\right)<\infty
$$

we get

$$
\int_{t_{i}}^{t_{i}+1}\left(\int_{\mathbb{R} / \mathbb{Z}}\left|\mathfrak{V}^{\alpha}\left(c_{t}\right)+\lambda \kappa_{c_{t}}\right|^{2} d x\right) d t=\int_{t_{i}}^{t_{i}+1}\left(\int_{\mathbb{R} / \mathbb{Z}}\left|\partial_{t} c_{t}\right|^{2} d x\right) d t \stackrel{i \rightarrow \infty}{\longrightarrow} 0
$$

Since our uniform estimates further imply

$$
\left|\frac{d}{d t} \int_{\mathbb{R} / \mathbb{Z}}\right| \mathfrak{V}^{\alpha}\left(c_{t}\right)+\left.\lambda \kappa_{c_{t}}\right|^{2} d x \mid \leq C
$$


for a constant $C<\infty$ independent of time, we deduce

$$
\int_{\mathbb{R} / \mathbb{Z}}\left|\mathfrak{V}^{\alpha}\left(c_{t}\right)+\lambda \kappa_{c_{t}}\right|^{2} d x \stackrel{t \rightarrow \infty}{\longrightarrow} 0
$$

which shows that $c_{\infty}$ is a critical point of $E^{\alpha}+\lambda L$.

Using the Łojasiewicz-Simon gradient inequality again we get

$$
\left(E\left(c_{\infty}\right)-E\left(c_{M}\right)\right)^{1-\theta} \leq C\left(\int_{\mathbb{R} / \mathbb{Z}}\left|V c_{\infty}\right|^{2}\left|c_{\infty}^{\prime}\right| d x\right)^{1 / 2}=0
$$

and hence $E\left(c_{\infty}\right)=E\left(c_{M}\right)$.

To get convergence of the complete flow, we repeat the estimates that led to (4.14) with $c_{\infty}$ in place of $c_{M}$ to get

$$
\left\|\tilde{c}_{t}-c_{\infty}\right\|_{C^{\beta}\left(\mathbb{R} / \mathbb{Z}, \mathbb{R}^{n}\right)} \leq C\left\|\tilde{c}_{t_{i}}-c_{\infty}\right\|_{C^{\beta}}^{\theta \sigma}
$$

for all $t \geq t_{i}$. So the complete flow converges in $C^{\beta}$ and hence by interpolation in $C^{\infty}$ to $c_{\infty}$

Proof of Theorem 1.2 Due to Lemma 9.1 for all $c \in C^{\beta}\left(\mathbb{R} / \mathbb{Z}, \mathbb{R}^{n}\right)$ with $\left\|c-c_{M}\right\|_{C^{\beta}} \leq$ $\varepsilon$ there is a diffeomorphism $\phi_{c}$ and a vector field $N_{c} \in C^{\beta}\left(\mathbb{R} / \mathbb{Z}, \mathbb{R}^{n}\right)$ normal to $c_{M}$ such that

$$
c \circ \phi_{c}=c_{M}+N_{c}
$$

and

$$
\left\|N_{c}\right\|_{C^{\beta}} \leq C\left\|c-c_{M}\right\|_{C^{\beta}}
$$

if $\varepsilon>0$ is small enough.

For $c \in C^{2+\alpha}$ with

$$
\left\|c-c_{M}\right\|_{C^{2+\alpha}} \leq \varepsilon
$$

let $\left(N_{t}\right)_{t \in[0, \tilde{T})}$ be the maximal solution of

$$
\left\{\begin{array}{l}
\partial_{t}^{\perp}\left(c_{M}+N_{t}\right)=H^{\alpha}\left(c_{M}+N_{t}\right)+\lambda \kappa_{c_{M}+N_{t}} \\
N_{0}=N_{c} .
\end{array}\right.
$$

Then $\tilde{T} \leq T$ and for all $t \in[0, \tilde{T})$ there are diffeomorphisms $\phi_{t}$ such that $c_{t}=$ $\left(c_{M}+N_{t}\right)\left(\phi_{t}\right)$. Hence $N_{t}$ satisfies all the assumptions of Theorem 4.2 if $\varepsilon$ is small enough and thus $\infty=\tilde{T}$. From $\tilde{T} \leq T$ we deduce $T=\infty$. 


\subsection{Completion of the Proof of Theorem 1.1}

It is only left to show that we get convergence of the flow without applying translations from the smooth subconvergence of the re-parameterized and translated curves we get from Sect. 3.4.

Let $\tilde{c}_{t}$ be the re-parameterizations of $c_{t}$ and let $t_{i} \rightarrow \infty$ and $p_{i} \in \mathbb{R}^{n}$ be such that the curves $\tilde{c}\left(t_{i}\right)-p_{i}$ converge smoothly to a curve $\tilde{c}_{\infty}$ parameterized by arc length. Due to the smooth convergence, the data $\tilde{c}_{t_{i}}-p_{i}$ satisfies all the assumptions of Theorem 1.2 for $i$ large enough and $c_{M}=c_{\infty}$. Hence, the statement follows directly from the conclusions of Theorem 1.2.

Acknowledgements Open access funding provided by Paris Lodron University of Salzburg.

Open Access This article is distributed under the terms of the Creative Commons Attribution 4.0 International License (http://creativecommons.org/licenses/by/4.0/), which permits unrestricted use, distribution, and reproduction in any medium, provided you give appropriate credit to the original author(s) and the source, provide a link to the Creative Commons license, and indicate if changes were made.

\section{Appendix: Analytic functions on Banach spaces}

We briefly prove some lemmata about analytic functions on Banach spaces. A thorough discussion of this subject can be found in [18, Chapter 2, Section 3].

Definition 5.1 (Analytic operator) Let $\left(X,\|\cdot\|_{X}\right),\left(Y,\|\cdot\|_{Y}\right)$ be real Banach spaces. A function $f \in C^{\infty}(A, Y), A \subset X$ open, is called real analytic if for every $a \in A$ there is a open neighborhood $U$ of $a$ in $X$ and a constant $C<\infty$ such that

$$
\left\|D^{m} f(x)\right\| \leq C^{m} m ! \quad \forall m \in \mathbb{N}, x \in U
$$

In this context $\|\cdot\|$ denotes the operator norm. The next lemmata show how to construct analytic functions:

Lemma 5.2 Let $g: U \rightarrow \mathbb{R}^{l}$ be a real analytic function, $U \subset \mathbb{R}^{n}$ be an open subset, and let $V \subset C^{k, \alpha}\left(\mathbb{R} / \mathbb{Z}, \mathbb{R}^{n}\right)\left(k \in \mathbb{N}_{0}, \alpha \in(0,1)\right)$ be an open subset such that $\operatorname{im} f \subset U$ for all $f \in V$. Then

$$
\begin{array}{r}
T: V \rightarrow C^{k, \alpha}\left(\mathbb{R} / \mathbb{Z}, \mathbb{R}^{l}\right) \\
x \rightarrow g \circ x
\end{array}
$$

defines a real analytic function.

Proof Let $f_{0} \in V$. Since im $f_{0}$ is a compact subset of the open set $U$ there is an $\varepsilon>0$ such that $K_{\varepsilon}:=\bigcup_{y \in \operatorname{im~} f_{0}} \overline{B_{\varepsilon}(y)} \subset U$.

Since $g$ is real analytic and $K_{\varepsilon} \subset U$ is compact, there is a constant $C<\infty$ such that

$$
\left\|D^{m} g(y)\right\| \leq C^{m} m !, \quad \forall m \in \mathbb{N}, y \in K_{\varepsilon} .
$$


As

$$
D^{m} T(y)\left(h_{1}, \ldots, h_{m}\right)=D^{m} g(y)\left(h_{1}, \ldots, h_{m}\right)
$$

(can easily be deduced from the Taylor expansion of $\mathrm{g}$ ) and since $C^{k, \alpha}(\mathbb{R} / \mathbb{Z})$ is a Banach algebra, we get

$$
\left\|D^{m} T(f)\right\| \leq(k+1) C^{m+k+1}(m+k+1) ! \leq \tilde{C}^{m} m !
$$

for all $f \in B_{\varepsilon}\left(f_{0}\right)=\left\{f_{0}+y \in C^{\alpha}:\|y\|_{C^{k, \alpha}} \leq \varepsilon\right\}$ where

$$
\tilde{C}:=\sup _{m \in \mathbb{N}_{0}}\left(\frac{(k+1) C^{m+k+1}(m+k+1) !}{m !}\right)^{1 / m} .
$$

Lemma 5.3 Let $\left(X,\|\cdot\|_{X}\right)$ and $\left(Y,\|\cdot\|_{Y}\right)$ be Banach spaces and assume that $T_{t} \in$ $C^{\omega}(X, Y)$ for $t \in I$ is such that the functions $t \rightarrow T_{t}$ are measurable and for all $a \in X$ there is a neighborhood $U$ of $a$ in $X$ such that

$$
\int_{I}\left(\sup _{y \in U}\left\|D^{m} T_{t}(y)\right\|\right) d t \leq C^{m} m !
$$

Then the mapping $T: X \rightarrow Y$ defined by

$$
T x=\int_{I} T_{t} x d t
$$

is real analytic.

Proof We want to show that

$$
D^{m} \operatorname{Tx}\left(h_{1}, \ldots, h_{m}\right)=\int_{I} D^{m} T_{t} x\left(h_{1}, \ldots, h_{m}\right) d t .
$$

from which we get that $T \in C^{\omega}(X, Y)$ using the estimates (5.1). In fact this follows from well-known facts about differentiation of parameter dependent integrals.

Remark 5.4 In the case that $Y=C^{k, \alpha}\left(\mathbb{R} / \mathbb{Z}, \mathbb{R}^{n}\right)$ we get from the fact that for $u \in \mathbb{R} / \mathbb{Z}$ the evalution mapping

$$
\begin{gathered}
F: Y \rightarrow \mathbb{R}^{n} \\
y \mapsto y(u)
\end{gathered}
$$

is a bounded linear operator form $Y$ to $\mathbb{R}^{n}$. Hence, the interchangability of the Bochner integral with linear operators (cf. for example [28, p. 134, Corollary 2]) tells us that 


$$
(T x)(u)=F(T x)=\int_{I} F\left(T_{t}\right) x d t=\int_{I}\left(T_{t}\right) x(u) d t
$$

i.e., the value of function $T x$ given by the Bochner integral at the point $u$ is equal to the Lebesque integral of the functions $T_{t}(u)$ evaluated at the point $u$.

\section{Estimates for a generalization of the multilinear Hilbert transform}

Lemma 6.1 Let $\alpha \in(0,1)$. For $1-\alpha \geq \beta>0, m \in \mathbb{N}$, and $t_{i} \in(0,1)$ the singular integral

$$
T\left(c_{1}, \ldots, c_{m}\right)(u):=p . v \cdot \int_{-\frac{1}{2}}^{\frac{1}{2}} \frac{1}{w|w|^{\alpha}} \prod_{i=1}^{m} c_{i}\left(u+t_{i} w\right) d w
$$

defines a bounded multilinear operator from $\left(C^{\alpha+\beta}(\mathbb{R} / \mathbb{Z}, \mathbb{R})\right)^{m}$ to $C^{\beta}(\mathbb{R} / \mathbb{Z}, \mathbb{R})$.

Proof For $u, v \in \mathbb{R} / \mathbb{Z}$ and $a \in\left(0, \frac{1}{2}\right)$ we get

$$
\begin{aligned}
\mid T\left(c_{1}, \ldots, c_{m}\right)(u)- & T\left(c_{1}, \ldots, c_{m}\right)(v) \mid \\
\leq & \int_{1 / 2 \geq|w| \geq a} \frac{1}{|w|^{\alpha+1}}\left|\prod_{i=1}^{m} c_{i}\left(u+t_{i} w\right)-\prod_{i=1}^{m} c_{i}\left(v+t_{i} w\right)\right| d w \\
& +\int_{a \geq|w|} \frac{1}{|w|^{\alpha+1}}\left|\prod_{i=1}^{m} c_{i}\left(u+t_{i} w\right)-\prod_{i=1}^{m} c_{i}(u)\right| d w \\
& +\int_{a \geq|w|} \frac{1}{|w|^{\alpha+1}}\left|\prod_{i=1}^{m} c_{i}\left(v+t_{i} w\right)-\prod_{i=1}^{m} c_{i}(v)\right| d w .
\end{aligned}
$$

Since $\left|t_{i}\right| \leq 1$, we obtain

$$
\left|\prod_{i=1}^{m} c_{i}\left(u+t_{i} w\right)-\prod_{i=1}^{m} c_{i}(u)\right| \leq m \prod_{i=1}^{m}\left\|c_{i}\right\|_{C^{\alpha+\beta}\left(\mathbb{R} / \mathbb{Z}, \mathbb{R}^{n}\right)}|w|^{\alpha+\beta}
$$

and hence, using the Hölder-continuity of the $c_{i}$,

$$
\begin{aligned}
& \left|T\left(c_{1}, \ldots, c_{m}\right)(u)-T\left(c_{1}, \ldots, c_{m}\right)(v)\right| \\
& \quad \leq m \prod_{i=1}^{m}\left\|c_{i}\right\|_{C^{\alpha+\beta}\left(\mathbb{R} / \mathbb{Z}, \mathbb{R}^{n}\right)}\left(\int_{\frac{1}{2} \geq|w| \geq a} \frac{1}{|w|^{1+\alpha}}|u-v|^{\alpha+\beta} d w+2 \int_{|w| \leq a} \frac{1}{|w|^{1-\beta}} d w\right) \\
& \quad \leq m \prod_{i=1}^{m}\left\|c_{i}\right\|_{C^{\alpha+\beta}\left(\mathbb{R} / \mathbb{Z}, \mathbb{R}^{n}\right)}\left(\frac{2}{\alpha} a^{-\alpha}|u-v|^{\alpha+\beta}+\frac{4}{\beta} a^{\beta}\right) .
\end{aligned}
$$


Choosing $a=|u-v|$ for $|u-v| \leq \frac{1}{2}$ we get

$$
\begin{aligned}
\mid T\left(c_{1}, \ldots, c_{m}\right)(u) & -T\left(c_{1}, \ldots, c_{m}\right)(v) \mid \\
& \leq C \prod_{i=1}^{m}\left\|c_{i}\right\|_{C^{\alpha+\beta}\left(\mathbb{R} / \mathbb{Z}, \mathbb{R}^{n}\right)}|u-v|^{\beta} .
\end{aligned}
$$

Lemma 6.2 For arbitrary $\beta>0, \alpha \in(0,1), m \in \mathbb{N}$, and $t_{i} \in(0,1)$ the singular integral

$$
T\left(c_{1}, \ldots c_{m}\right)(u):=p . v \cdot \int_{-\frac{1}{2}}^{\frac{1}{2}} \frac{1}{w|w|^{\alpha}} \prod_{i=1}^{m} c_{i}\left(u+t_{i} w\right) d w
$$

defines a bounded multilinear operator from $\left(h^{\alpha+\beta}(\mathbb{R} / \mathbb{Z}, \mathbb{R})\right)^{m}$ to $h^{\beta}(\mathbb{R} / \mathbb{Z}, \mathbb{R})$.

Proof First, let us note that it is enough to prove the statement for $\beta=\tilde{\beta}+n, n \in \mathbb{N}_{0}$, $1-\alpha \geq \tilde{\beta}>0$. One then uses real interpolation to prove the full statement. For $n=0$ the claim is the content of Lemma 6.1. So let us assume that the statement holds for $\beta=\tilde{\beta}+n$ as above. Let $\left(\tau_{h} f\right)(x):=f(x+h)$. Using the relation $\tau_{h}\left(T\left(c_{1}, \ldots, c_{m}\right)\right)=$ $T\left(\tau_{h}\left(c_{1}\right), \ldots, \tau_{h}\left(c_{m}\right)\right)$ and the multilinearity of $T$, the difference quotient can be written as

$$
\begin{aligned}
\frac{\tau_{h}\left(T\left(c_{1}, \ldots, c_{m}\right)\right)-T\left(c_{1}, \ldots, c_{m}\right)}{h} & =\frac{\left.T\left(\tau_{n}\left(c_{1}\right), \ldots, \tau_{h}\left(c_{m}\right)\right)-T\left(c_{1}, \ldots, c_{m}\right)\right)}{h} \\
& =\sum_{i=1}^{m} T\left(c_{1}, \ldots,\left(\tau_{h}\left(c_{i}\right)-c_{i}\right) / h, \ldots, \tau_{h}\left(c_{m}\right)\right) .
\end{aligned}
$$

Since

$$
\begin{aligned}
\left(\tau_{h}\left(c_{i}\right)-c_{i}\right) / h \stackrel{h \rightarrow 0}{\longrightarrow} c_{i}^{\prime} & \text { in } C^{\beta} \\
\tau_{h}\left(c_{i}\right) \stackrel{h \rightarrow 0}{\longrightarrow} c_{i} & \text { in } C^{\beta}
\end{aligned}
$$

and $T$ a bounded linear operator from $C^{\beta}$ to $C^{\tilde{\beta}}$, we get

$$
\frac{\tau_{h}\left(T\left(c_{1}, \ldots, c_{m}\right)\right)-T\left(c_{1}, \ldots, c_{m}\right)}{h} \stackrel{h \rightarrow 0}{\longrightarrow} \sum_{i=1}^{m} T\left(c_{1}, \ldots, c_{i}^{\prime}, \ldots, c_{m}\right)
$$

in $C^{\beta}$. Hence, $T$ is a bounded multilinear mapping from $\left(C^{\alpha+\beta+1}\right)^{m}$ to $C^{\beta+1}$.

Remark 6.3 Let us state a simple extension of Lemma 6.2. Given a multilinear form $M: \mathbb{R}^{n} \times \cdots \times \mathbb{R}^{n} \rightarrow \mathbb{R}^{k}, 1>\alpha>0, \beta>0, m \in \mathbb{N}$, and $t_{i} \in(0,1)$ the singular integral 


$$
\left.T\left(c_{1}, \ldots c_{m}\right)(u):=p . v \cdot \int_{-\frac{1}{2}}^{\frac{1}{2}} \frac{1}{w|w|^{\alpha}} M\left(c_{1}\left(u+t_{1} w\right), \ldots, c_{m}\left(u+t_{m} w\right)\right)\right) d w
$$

defines a bounded multilinear operator from $\left(h^{\alpha+\beta}(\mathbb{R} / \mathbb{Z}, \mathbb{R})\right)^{m}$ to $h^{\beta}\left(\mathbb{R} / \mathbb{Z}, \mathbb{R}^{n}\right)$.

This can be deduced by plugging

$$
M\left(c_{1}\left(u+t_{1} w\right), \ldots, c_{m}\left(u+t_{m} w\right)\right)=\sum_{j_{1}, \ldots, j_{m}=1}^{n} M\left(e_{i_{1}}, \ldots, e_{j_{m}}\right) \Pi_{i=1}^{m}\left\langle c_{j}\left(u+t_{i} w\right), e_{j_{i}}\right\rangle
$$

into the definition of $T$, where $e_{1}, \ldots e_{n}$ is the standard basis of $\mathbb{R}^{n}$, and by applying Lemma 6.2 to all the coordinates of the resulting summands.

\section{Facts about the functional $Q^{\alpha}$}

In this section we prove a commutator inequality that we use as a substitute for the Leibniz rule for $Q^{\alpha}$.

Lemma 7.1 (Leibniz rule for $Q^{\alpha}$ ) For $f \in C^{\alpha+\beta}\left(\mathbb{R} / \mathbb{Z}, \mathbb{R}^{n}\right), g \in C^{\alpha+\beta+1}\left(\mathbb{R} / \mathbb{Z}, \mathbb{R}^{n}\right)$ and $\beta>0$ we have

$$
\left\|Q^{\alpha}(f g)-Q^{\alpha}(f) g\right\|_{C^{\beta}} \leq C(\alpha, \beta)\|f\|_{C^{\alpha+\beta}}\|g\|_{C^{\alpha+1+\beta}} .
$$

Proof We have

$$
\begin{aligned}
& \left(Q^{\alpha}(f g)-Q^{\alpha}(f) g\right)(u) \\
& \text { p.v. } \int_{[-1 / 2,1 / 2]}\left\{2 \frac{f(u+w) g(u+w)-f(u) g(u)-w\left(f^{\prime}(u) g(u)-f(u) g^{\prime}(u)\right)}{w^{2}}\right. \\
& \left.-\left(f^{\prime \prime}(u) g(u)+2 g^{\prime}(u) f^{\prime}(u)+f(u) g^{\prime \prime}(u)\right)\right\} \frac{d w}{|w|^{\alpha}} \\
& \text { - p.v. } \int_{[-1 / 2,1 / 2]}\left\{2 \frac{f(u+w) g(u)-f(u) g(u)-w f^{\prime}(u) g(u)}{w^{2}}-f^{\prime \prime}(u) g(u)\right\} \frac{d w}{|w|^{\alpha}} \\
& =\int_{[-1 / 2,1 / 2]}\left\{2 \frac{(f(u+w)-f(u))(g(u+w)-g(u))}{w^{2}}-2 f^{\prime}(u) g^{\prime}(u)\right. \\
& \left.+f(u)\left(2 \frac{g(u+w)-g(u)-w g^{\prime}(u)}{w^{2}}-g^{\prime \prime}(u)\right)\right\} \frac{d w}{|w|^{\alpha}} \\
& =2 \text { p.v. } \int_{[-1 / 2,1 / 2]}\left\{\frac{(f(u+w)-f(u))(g(u+w)-g(u))}{w^{2}}-f^{\prime}(u) g^{\prime}(u)\right\} \frac{d w}{|w|^{\alpha}} \\
& +\left(f Q^{\alpha}(g)\right)(u) \text {. }
\end{aligned}
$$


Taylor expansion yields

$$
\begin{gathered}
\frac{f(u+w)-f(u)}{w}=f^{\prime}(u)+w \int_{0}^{1}(1-t) f^{\prime \prime}(u+t w) d t \\
\frac{g(u+w)-g(u)}{w}=g^{\prime}(u)+w \int_{0}^{1}(1-t) g^{\prime \prime}(u+t w) d t
\end{gathered}
$$

and hence the first term in the last row of Eq. (7.1) can be written as

$$
\begin{aligned}
2 \int_{[-1 / 2,1 / 2]}\{ & \left.\frac{(f(u+w)-f(u))(g(u+w)-g(u))}{w^{2}}-f^{\prime}(u) g^{\prime}(u)\right\} \frac{d w}{|w|^{\alpha}} \\
& =2 f^{\prime}(u) \int_{[-1 / 2,1 / 2]} \frac{\int_{0}^{1}(1-t) g^{\prime \prime}(u+t w) d t}{w} \frac{d w}{|w|^{\alpha-2}} \\
& +2 g^{\prime}(u) \int_{[-1 / 2,1 / 2]} \frac{\int_{0}^{1}(1-t) f^{\prime \prime}(u+t w) d t}{w} \frac{d w}{|w|^{\alpha-2}} \\
& +2 \int_{[-1 / 2,1 / 2]} \int_{[0,1][0,1]}(1-t)(1-s) g^{\prime \prime}(u+t w) f^{\prime \prime}(u+s w) d t d s \frac{d w}{|w|^{\alpha-2}}
\end{aligned}
$$

It is an easy exercise to prove that the last term defines a bounded operator from $C^{2+\beta}$ to $C^{\beta}$ for all $\beta>0$. Using Lemma 6.2 to estimate the first two terms, we get

$$
\begin{array}{r}
\left\|Q^{\alpha}(f g)-Q^{\alpha}(f) g-g Q^{\alpha}(f)\right\|_{C^{\beta}} \\
\leq C\left(\left\|f^{\prime}\right\|_{C^{\beta}}\left\|g^{\prime \prime}\right\|_{C^{\alpha-2+\beta}}+\left\|g^{\prime}\right\|_{C^{\beta}}\left\|f^{\prime \prime}\right\|_{C^{\alpha-2+\beta}}+\left\|f^{\prime \prime}\right\|_{C^{\beta}}\left\|g^{\prime \prime}\right\|_{C^{\beta}}\right)
\end{array}
$$

and hence

$$
\left\|Q^{\alpha}(f g)-Q^{\alpha}(f) g-f Q^{\alpha}(g)\right\|_{C^{\beta}} \leq\|f\|_{C^{\alpha+\beta}}\|g\|_{C^{\alpha+\beta}} .
$$

Together with the fact that $Q$ defines a bounded linear operator from $C^{\alpha+1+\beta}$ to $C^{\beta}$ this proves the estimate.

\section{Interpolation and Leibniz rule for fractional Sobolev spaces}

For the convenience of the reader we present mutliplicative Gagliardo-NirenbergSobolev inequalities and a Leibniz rule in fractional Sobolev spaces and nonhomogeneous Besov spaces that we used in this text. We use the notation

$$
B_{q}^{s, p}
$$


where $s$ denotes the order of differentiation and $p$ the integrability. So for $s \in(0,1)$, $p, q \in(1, \infty)$ the space $B_{p}^{s, q}(\mathbb{R} / \mathbb{Z})$ consists of all $L^{p}$ - functions satisfying

$$
\lfloor f\rfloor_{B_{q}^{s, p}}:=\left(\int_{0}^{\frac{1}{2}}\left(\frac{\|f(\cdot)-f(\cdot+w)\|_{L^{p}(\mathbb{R} / \mathbb{Z})}}{w^{s}}\right)^{q} \frac{d w}{w}\right)^{\frac{1}{q}} .
$$

Theorem 8.1 (Gagliardo-Nirenberg-Sobolev Inequality) For $p \in[2, \infty), q \in$ $[1, \infty)$ and $s_{1}, s_{2}, s_{3} \in[0, \infty)$ with $s_{1}-1 / 2 \leq s_{2}-1 / p \leq s_{3}-1 / 2$ there is $a$ $C=C\left(s_{1}, s_{2}, s_{3}, p, q\right)<\infty$ such that

$$
\|u\|_{B_{q}^{s_{2}, p}} \leq C\|u\|_{W^{s_{3}, 2}}^{\theta}\|u\|_{W^{s_{1}, 2}}^{1-\theta}
$$

for all smooth functions $u \in C^{\infty}(\mathbb{R} / \mathbb{Z})$ where $\theta:=\left(s_{2}-s_{1}-1 / p+1 / 2\right) /\left(s_{3}-s_{1}\right)$. Especially,

$$
\|u\|_{W^{s_{2}, p}} \leq C\|u\|_{W^{s_{3}, 2}}^{\theta}\|u\|_{W^{s_{1}, 2}}^{1-\theta} .
$$

Proof Using [22, Proposition 1.20], it is enough to show that the real interpolation space $\left(W^{s_{1}, 2}, W^{s_{2}, 2}\right)_{\theta, 1}$ is continuously embedded in $B_{q}^{s_{2}, p}$. From [27, Section 1.6.7], we get $\left(W^{s_{1}, 2}, W^{s_{3}, 2}\right)_{\theta, 1}=B_{1}^{\tilde{s}, 2}$ where $\tilde{s}:=\theta s_{3}+(1-\theta) s_{1} \geq s_{2}$. Since $p \geq 2$, we get $\tilde{s} \geq s_{2}$. As furthermore $\tilde{s}-1 / 2=s_{2}-1 / p$, the Sobolev embedding tells us that

$$
B_{1}^{\tilde{s}, 2} \subset B_{1}^{s_{2}, p} \subset B_{q}^{s_{2}, p}
$$

and especially

$$
B_{1}^{\tilde{s}, 2} \subset B_{1}^{s_{2}, p} \subset B_{p}^{s_{2}, p}=W^{s_{2}, p}
$$

which completes the proof.

The following product rule goes back to Coifman and Meyer [11].

Lemma 8.2 (Leibniz Rule, cf. [11]) Let $p_{i}, q_{i}, r \in(1, \infty)$, be such that $\frac{1}{p_{i}}+\frac{1}{q_{i}}=\frac{1}{r}$, for $i=1,2$ and $s>0$. Then

$$
\|f \cdot g\|_{W^{s, r}} \leq C\left(\|f\|_{L^{p_{1}}}\|g\|_{W^{s, q_{1}}}+\|f\|_{W s, p_{2}}\|g\|_{L q_{2}}\right) .
$$

We also refer to Runst and Sickel [25, Lem. 5.3.7/1 (i)].

Let us conclude this section with an elementary proof of the following well-known interpolation estimate. For a subset $A \subset \mathbb{R}$ and a function $f: A \rightarrow \mathbb{R}$ we set

$$
\text { höl } 1_{\alpha, A} f=\sup _{x, y \in A, x \neq y} \frac{|f(x)-f(y)|}{|x-y|} \text {. }
$$

Lemma 8.3 For all $k>m, \beta \in(0,1)$ there is a constant $C<\infty$ such that

$$
h \ddot{o} l_{\beta, \mathbb{R}} f^{(m)} \leq\left\|f^{(k)}\right\|_{L^{\infty}(\mathbb{R})}^{1-\sigma}\|f\|_{L^{2}(\mathbb{R})}^{\sigma}
$$


for all $f \in C_{l o c}^{k}(\mathbb{R})$ where $\sigma=\frac{\alpha-\beta}{\alpha+\frac{1}{2}}$. Furthermore,

$$
\|f\|_{C^{m, \beta}(\mathbb{R} / \mathbb{Z})} \leq\left\|f^{(k)}\right\|_{L^{\infty}(\mathbb{R} / \mathbb{Z})}^{1-\sigma}\|f\|_{L^{2}(\mathbb{R} / \mathbb{Z})}^{\sigma}
$$

Proof Ehrling's lemma together with the estimate $\|f\|_{C^{k}} \leq C\left(\left\|f^{(k)}\right\|_{L^{\infty}}+\|f\|_{L^{\infty}}\right)$ tells us that for every $\varepsilon>0$ there is an $C(\varepsilon)<\infty$ such that

$$
\left\|f^{(m)}\right\|_{C^{\beta}\left(B_{1}(0)\right)} \leq \varepsilon\left(\left\|f^{(k)}\right\|_{L^{\infty}\left(B_{1}(0)\right)}+\|f\|_{L^{\infty}\left(B_{1}(0)\right)}+C(\varepsilon)\|f\|_{L^{2}\left(B_{1}(0)\right)}\right.
$$

which for $\varepsilon=\frac{1}{2}$ implies

$$
\operatorname{höl}_{\beta, B_{1}(0)} f^{(m)} \leq\left\|f^{(k)}\right\|_{L^{\infty}\left(B_{1}(0)\right)}+2 C(1 / 2)\|f\|_{L^{2}\left(B_{1}(0)\right)} .
$$

Scaling this inequality yields

$$
r^{\beta+m} \operatorname{höl}_{\beta, B_{r}(0)} f^{(m)} \leq r^{k}\left\|f^{(k)}\right\|_{L^{\infty}\left(B_{r}(0)\right)}+C r^{-\frac{1}{2}}\|f\|_{L^{2}\left(B_{r}(0)\right)}
$$

for all $r>0$ and hence we get covering $\mathbb{R}$ by balls of radius $r \in[0,1 / 2]$

$$
\operatorname{höl}_{\beta, \mathbb{R}} f^{(m)} \leq C\left(r^{k-\beta-m}\left\|f^{(k)}\right\|_{L^{\infty}(\mathbb{R})}+r^{-\beta-m-\frac{1}{2}}\|f\|_{L^{2}(\mathbb{R})}\right) .
$$

Hence, we get the first estimate by choosing $r=\left(\frac{\left\|f^{k}\right\|_{L^{\infty}(\mathbb{R})}}{\|f\|_{L^{2}(\mathbb{R})}}\right)^{\frac{1}{k+1 / 2}}$.

For the second estimate, we observe that Ehrling's lemma gives

$$
\|f\|_{C^{m}(\mathbb{R} / \mathbb{Z})} \leq \frac{1}{2}\|f\|_{C^{m, \beta}(\mathbb{R} / \mathbb{Z})}+C\|f\|_{L^{2}(\mathbb{R} / \mathbb{Z})}
$$

and hence

$$
\|f\|_{C^{m}(\mathbb{R} / \mathbb{Z})} \leq h \operatorname{hol}_{\beta, \mathbb{R}} f^{(m)}+2 C\|f\|_{L^{2}(\mathbb{R} / \mathbb{Z})} .
$$

Combining this with the first estimate gives

$$
\begin{aligned}
\|f\|_{C^{m, \beta}(\mathbb{R} / \mathbb{Z})} & \leq C h \operatorname{hol}_{\beta, \mathbb{R}} f^{(m)}+C\|f\|_{L^{2}(\mathbb{R} / \mathbb{Z})} \\
& \leq C\|f\|_{C^{k}(\mathbb{R} / \mathbb{Z})}^{1-\sigma}\|f\|_{L^{2}(\mathbb{R} / \mathbb{Z})}^{\sigma}+C\|f\|_{L^{2}(\mathbb{R} / \mathbb{Z})} \\
& \leq C\|f\|_{C^{k}(\mathbb{R} / \mathbb{Z})}^{1-\sigma}\|f\|_{L^{2}(\mathbb{R} / \mathbb{Z})}^{\sigma}
\end{aligned}
$$

\section{Normal graphs}

The following lemma is used in the proofs of Lemma 4.1 and Theorem 3.1. 
Lemma 9.1 Let $c_{0} \in C_{i, r}^{\infty}\left(\mathbb{R} / \mathbb{Z}, \mathbb{R}^{n}\right)$ and $W=C^{s}(\mathbb{R} / \mathbb{Z})$ or $W=H^{s+\frac{1}{2}}(\mathbb{R} / \mathbb{Z})$ for some $s>1$. Then there is an $\varepsilon>0$ such that for all $c \in W$ with

$$
\left\|c-c_{0}\right\|_{W} \leq \varepsilon
$$

there is a re-parameterization $\phi$ and a function $N \in C^{\alpha}\left(\mathbb{R} / \mathbb{Z}, \mathbb{R}^{n}\right)$ normal to $c_{0}$ such that

$$
c \circ \phi=c_{0}+N
$$

and

$$
\|N\|_{W} \leq C\left\|c-c_{0}\right\|_{W}
$$

Proof Note that in the case $W=H^{s+\frac{1}{2}}$, still $W$ is embedded continuously in $C^{s}$ Let $U$ be an open neighborhood of $c_{0}$ such that there is a nearest point retract $r_{U}$, i.e. a $C^{\infty}$ function $r_{U}: U \rightarrow \mathbb{R} / \mathbb{Z}$ such that

$$
c_{0} \circ r_{U} \circ c_{0}=c_{0}
$$

and

$$
\left|c_{0}\left(r_{U}(p)\right)-p\right|=\inf _{x \in \mathbb{R} / \mathbb{Z}}\left|c_{0}(x)-p\right| \quad \forall p \in \mathbb{R} / \mathbb{Z}
$$

We set

$$
\psi_{c}(x)=r_{U}(c(x)) .
$$

Since $\psi_{c_{0}}=i d$ and the space of diffeomorphisms is open in $C^{s}$, the function $\psi_{c}$ is a diffeomorphism for sufficiently small $\varepsilon>0$. Furthermore, the mapping $c \rightarrow \psi_{c}$ is smooth from $C^{s}\left(\mathbb{R} / \mathbb{Z}, \mathbb{R}^{n}\right)$ to $C^{s}(\mathbb{R} / \mathbb{Z}, \mathbb{R} / \mathbb{Z})$ in the case that $W=C^{s}$ and from $H^{s+\frac{1}{2}}\left(\mathbb{R} / \mathbb{Z}, \mathbb{R}^{n}\right)$ to $H^{s+\frac{1}{2}}(\mathbb{R} / \mathbb{Z}, \mathbb{R} / \mathbb{Z})$ in the case that $W=H^{s+\frac{1}{2}}$. We set

$$
\phi_{c}=\psi_{c}^{-1}
$$

and

$$
N_{c}(x)=c \circ \psi_{c}^{-1}-c_{0} .
$$

The estimate in the lemma now follows from the fact that $c \rightarrow N_{c}$ is a smooth function for $W$ to $W$ in neighborhood of $c_{0}$ with $N_{c_{0}}=0$.

\section{References}

1. Abrams, A., Cantarella, J., Fu, J.H.G., Ghomi, M., Howard, R.: Circles minimize most knot energies. Topology 42(2), 381-394 (2003)

2. Adams, R.A., Fournier, J.J.F.: Sobolev spaces, volume 140 of Pure and Applied Mathematics (Amsterdam), 2nd edn. Elsevier, Amsterdam (2003) 
3. Amann, H.: Nonhomogeneous linear and quasilinear elliptic and parabolic boundary value problems. In: Function Spaces, Differential Operators and Nonlinear Analysis (Friedrichroda, 1992), volume 133 of Teubner-Texte Math., pp. 9-126. Teubner, Stuttgart (1993)

4. Angenent, S.B.: Nonlinear analytic semiflows. Proc. R. Soc. Edinburgh Sect. A 115(1-2), 91-107 (1990)

5. Blatt, S.: Boundedness and regularizing effects of O'Hara's knot energies. J. Knot Theory Ramif. 21(1), 1250010,9 (2012)

6. Blatt, S.: The gradient flow of the Möbius energy near local minimizers. Calc. Var. Partial Differ. Equ. 43(3-4), 403-439 (2012)

7. Bourdaud, G.: Une algèbre maximale d'opérateurs pseudo-différentiels. Commun. Partial Differ. Equ. 13(9), 1059-1083 (1988)

8. Blatt, S., Reiter, P.: Stationary points of O'Hara's knot energies. Manuscr. Math. 140(1-2), 29-50 (2013)

9. Blatt, S., Reiter, P.: Towards a regularity theory for integral Menger curvature. Ann. Acad. Sci. Fenn. Math. 40(1), 149-181 (2015)

10. Chill, R.: On the Łojasiewicz-Simon gradient inequality. J. Funct. Anal. 201, 572-601 (2003)

11. Coifman, R.R., Meyer, Y.: Au delà des opérateurs pseudo-différentiels, volume 57 of Astérisque. Société Mathématique de France, Paris (1978). With an English summary

12. Dziuk, G., Kuwert, E., Schätzle, R.: Evolution of elastic curves in $\mathbb{R}^{n}$ : existence and computation. SIAM J. Math. Anal. 33(5), 1228-1245 (2002). (electronic)

13. Di Nezza, E., Palatucci, G., Valdinoci, E.: Hitchhiker's guide to the fractional Sobolev spaces. Bull. Sci. Math. 136(5), 521-573 (2012)

14. Freedman, M.H., He, Z.-X., Wang, Z.: Möbius energy of knots and unknots. Ann. Math. (2) 139(1), 1-50 (1994)

15. Gilbarg, D., Trudinger, N.S.: Elliptic partial differential equations of second order: Classics in Mathematics. Springer, Berlin (2001). (Reprint of the 1998 edition)

16. He, Z.: A formula for the non-integer powers of the Laplacian. Acta Math. Sin. (Engl. Ser). 15(1), 21-24 (1999)

17. He, Z.-X.: The Euler-Lagrange equation and heat flow for the Möbius energy. Commun. Pure Appl. Math. 53(4), 399-431 (2000)

18. Hille, E., Phillips, R.S.: Functional analysis and semi-groups. Colloquium Publications, vol. 31, 808 p. American Mathematical Society (AMS), Providence, R.I (1957 rev. ed.)

19. Kusner, R.B., Sullivan, J.M.: Möbius energies for knots and links, surfaces and submanifolds. In: Geometric Topology (Athens, GA, 1993), Volume 2 of AMS/IP Stud. Adv. Math., pp. 570-604. Amer. Math. Soc., Providence, RI (1997)

20. Lin, C.-C., Schwetlick, H.R.: On a flow to untangle elastic knots. Calc. Var. Partial Differ. Equ. 39(3-4), 621-647 (2010)

21. Lunardi, A.: Analytic semigroups and optimal regularity in parabolic problems. Progress in Nonlinear Differential Equations and their Applications, 16. Birkhäuser, Basel (1995)

22. Lunardi, A.: Interpolation theory. Appunti. Scuola Normale Superiore di Pisa (Nuova Serie). (Lecture Notes. Scuola Normale Superiore di Pisa (New Series)), 2nd edn. Edizioni della Normale, Pisa (2009)

23. O'Hara, J.: Energy Functionals of Knots. Topology Hawaii (Honolulu. HI, 1990), pp. 201-204. World Science Publication, River Edge (1992)

24. Reiter, P.: Repulsive knot energies and pseudodifferential calculus for O'Hara's knot energy family $E^{(\alpha)}, \alpha \in[2,3)$. Math. Nachr. 285(7), 889-913 (2012)

25. Runst, T., Sickel, W.: Sobolev spaces of fractional order, Nemytskij operators, and nonlinear partial differential equations. de Gruyter Series in Nonlinear Analysis and Applications, vol. 3. Walter de Gruyter and Co., Berlin (1996)

26. Triebel, H.: Theory of function spaces. Monographs in Mathematics, vol. 78. Birkhäuser, Basel (1983)

27. Triebel, H.: Theory of function spaces. II, volume 84 of Monographs in Mathematics. Birkhäuser, Basel (1992)

28. Yosida, K.: Functional Analysis. Springer Nature (1995) 